\title{
Silencing hyper-excitable neurons as a treatment for status epilepticus and temporal lobe epilepsy
}

\author{
Deblina Dey \\ Charlottesville, Virginia \\ M.S. Biology, University of Vermont \\ M.Sc. Zoology, University of Calcutta, India \\ B.Sc. Zoology Honors, University of Calcutta, India
}

A Dissertation presented to the Graduate Faculty of the University of Virginia in Candidacy for the Degree of Doctor of Philosophy

\author{
Neuroscience Graduate Program \\ Department of Pharmacology
}

University of Virginia

December, 2013 


\section{Thesis Abstract}

Epilepsy is a neurological disorder affecting millions of people around the globe. It is characterized by recurrent, spontaneous seizures. Seizures are broadly classified into partial seizures and generalized seizures. Temporal lobe epilepsy (TLE) is the most common form of adult focal epilepsy. Almost one third of TLE suffering patients are non-responsive to anti-epileptic drugs. The last resort for these patients is surgery. Surgery comes with serious side effects and also does not promise complete cure from the disease. Status epilepticus (SE) is a medical emergency which is characterized by a seizure lasting for least 5 minutes. It affects 110,000 to 160,000 people annually in United States alone and results in 22,000-42000 deaths annually. Anti-epileptic drugs fail to treat status epilepticus in almost $23-43 \%$ of suffering patients at some point. This thesis focused on finding an alternative gene therapy treatment to treat seizures focusing mainly on SE and TLE. Recombinant adeno-associated virus mediated gene have been in development for Alzheimer's and Parkinson's disease. Recently adeno-associated virus serotype 1 mediated gene therapy for lipoprotein lipase deficiency disorder has entered the European clinics. This study investigates the effect of adeno-associated virus mediated expression of constitutively-active potassium leak channel Trek-1 (Trek-M) on SE induced by intra-peritoneal injection of pilocarpine. The results have demonstrated that expression of a constitutively active potassium channel in rodent brain prior to pilocarpine insult causes a significant reduction in duration of SE. Significant less amount of neuronal death was observed in the brain regions like entorhinal cortex, CA3, CA1 in rats preinjected with TREK-M. This finding suggested that TREK-M preinjection prevented neuronal death in the brain regions where it was injected. We have made the 
first doxycycline regulated AAV targeting vector which can be turned on by addition of doxycycline in the diet. Doxycycline regulated expression of TREK-M showed promising results in significantly decreasing the normalized seizure frequency of chronic seizures in lithium pilocarpine rat model of epilepsy. This study of gene therapy approach to silence hyper-excitable neurons to treat seizures will pave the way for developing gene therapy to treat seizures in patients suffering from refractory convulsive status epilepticus and temporal lobe epilepsy. 


\section{Table of Contents}

Chapter 1: Introduction

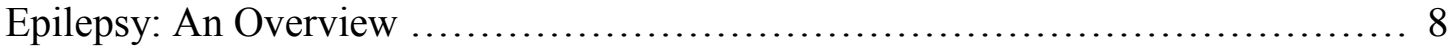

Available treatments for SE and TLE....................................... 12

Brain structures involved in temporal lobe epilepsy............................ 14

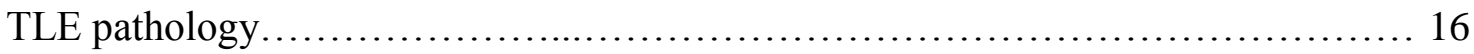

The use of gene therapy for the treatment of epilepsy........................ 18

Gene of choice-Trek potassium channel..................................... 20

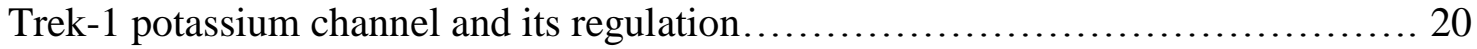

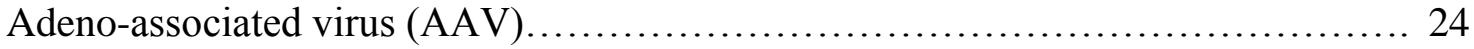

Development of Dox-On targeting vector................................ 27

GABRA4 promoter.................................................. 28

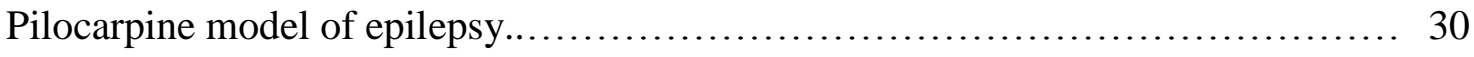

Specific Aims....................................................... 31

Chapter 2: A potassium leak channel silences hyper-excitable neurons and ameliorates status epilepticus

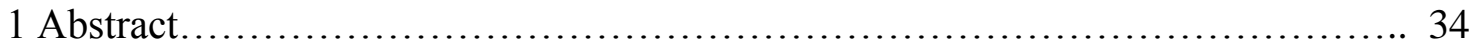

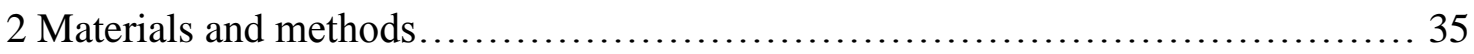

2.1 Transfection.......................................................... 35

2.2 Cloning of TREK-M and viral constructs............................... 35

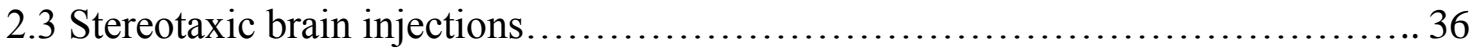

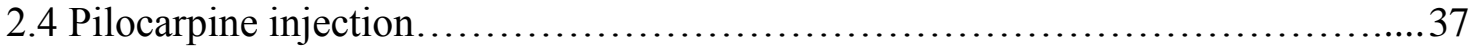

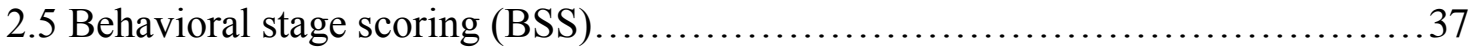

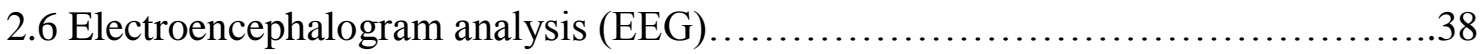


2.7 Tissue preparation for Fluoro-Jade C staining ............................. 40

2.8 Fluoro-Jade C staining .................................................. 40

2.9 Image acquisition and data analysis....................................... 41

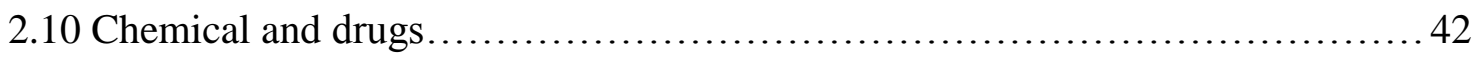

2.11 Adeno-associated virus constructs.........................................42

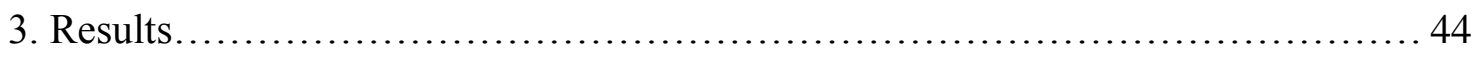

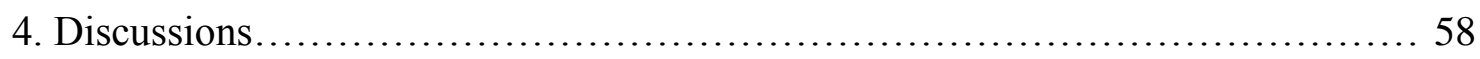

Chapter 3: Testing drug-regulated expression of TREK-M on chronic seizures in the lithium-pilocarpine model of epileptic rats

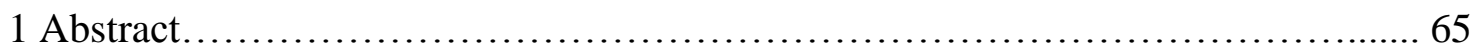

2 Materials and methods

2.1 Adeno-associated virus construct.........................................6 66

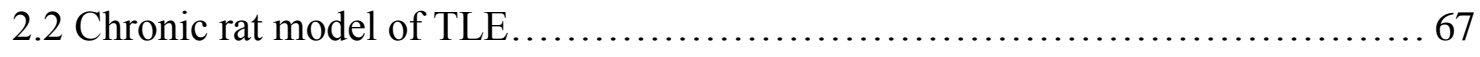

2.3 Stereotaxic brain injection................................................. 68

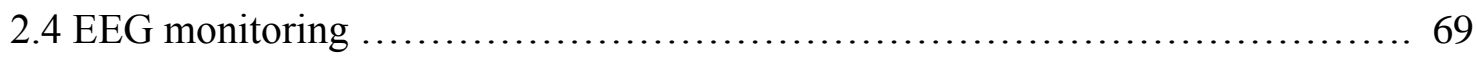

2.5 Data analysis............................................................... 70

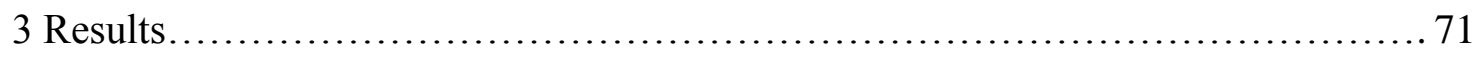

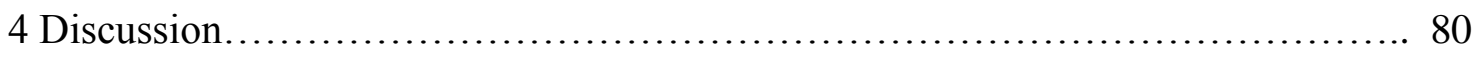

Chapter 4: Summary and future directions ................................. 85

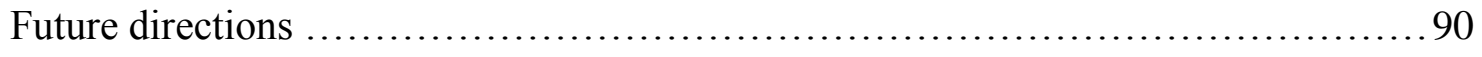

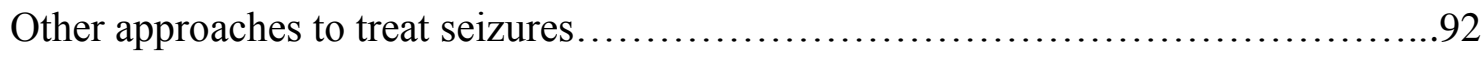

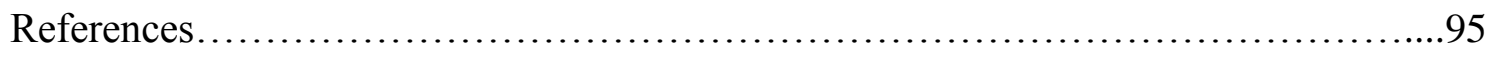




\section{List of figures}

Figure 1 Regions of hippocampal formation 16

Figure 2 EEG traces showing the different electrographic stages obtained from a pilocarpine injected epileptic rat preinjected with AAV-TREK-M virus.

Figure 4 Fluorescent measurement of yellow fluorescent protein Venus by various combinations of the GABRA4 promoter.

Figure 5 Specificity of GABRA4 promoter in neuronal and non-neuronal cells....

Figure 6 Validation of AAV expression after intraparenchymal injection into the hippocampal CA3 layer and entorhinal cortex (EC)....

Figure 7 Effect of pre-injection of AAV-Ctrl and AAV-TREK-M virus in pilocarpine induced status epilepticus in rats 52

Figure 8 Effect of preinjection of AAV-CTRL and AAV-TREKM on neuronal death in pilocarpine induced epileptic rats. 54

Figure 9 Changes in the number if dead neurons in the EC, CA3, CA1 and DG regions of AAV5-TREKM preinjected rats compared to that if AAV-CTRL preinjected rats... 56

Figure 10 Time-line of lithium pilocarpine chronic epilepsy model of rat 67

Figure 11 AAV5-SmonCeiT construct used for in vivo experiments. 70 
Figure 12 Validation of doxycycline regulated expression of AAV5-SmonCeiT virus after intraparenchymal injection into the CA3and entorhinal cortex (EC) of chronic female epileptic rats.................................................. 72

Figure 13 Seizure diaries corresponding to rat DP25, DP24, DP23 and DP22 ..... 75

Figure 14 Effect of AAV5-SmonCeiT on normalized seizure frequency of rats...... 77

Figure 15 Expression of doxycycline regulated mCherry expression of rat DP25... 79

Figure 16 Altered structure of the dentate granule cell layer in DP25............. 84

Appendix figure 1 Mutations in TREK-1 increase $\mathrm{K}+$ leak currents, lower resting membrane potential and decrease input resistance........................... 113

Appendix figure 2 TREK-M is not regulated by intracellular acidosis or second messenger signaling cascades............................................. 115

Appendix figure 3 TREK-M reduces spontaneous firing of hyperactive hippocampal neurons................................................................... 117

Appendix figure 4 Validation of SmonCeiT construct in vitro...................... 119 


\section{Epilepsy: An overview}

Epilepsy is one of the most common neurologic disorders. 70 million people worldwide suffers from this disorder (Brodie, Schachter et al. 2012). It has been reported that the total occurrence of seizures is about $1.7 \%$ up to age 50 and $3.4 \%$ by age 80 (Engel 2013). It has been shown than $10 \%$ of the population within a lifespan of 80 years will suffer from this disorder (Engel 2013).

Epilepsy is a neurological disorder which is characterized by spontaneous recurrent seizures. An epileptic seizure is defined as transient, synchronized discharge of a group of neurons in brain (Fisher, Boas et al. 2005). Seizures are mainly caused when the balance between glutamatergic pathways mediated excitation and the GABA mediated inhibition in the brain is disturbed and the balance is tipped to excitation (Lothman, Rempe et al. 1995). A change in neuronal hyper-excitability can also be caused by change in expression and function of voltage-gated sodium channels. Studies in animal models of epilepsy have shown alteration in $\alpha$-subunits of $\mathrm{Na}_{\mathrm{v}} 1.1, \mathrm{Na}_{\mathrm{v}} 1.2, \mathrm{Na}_{\mathrm{v}} 1.3$ and $\mathrm{Na}_{\mathrm{v}} 1.6$ and other auxiliary subunits of sodium channels (Aronica, Yankaya et al. 2001; Ketelaars, Gorter et al. 2001; Blumenfeld, Lampert et al. 2009). Mutations in potassium channels also have been found to be associated with epilepsy. Mutations in KCNQ potassium channels are found in benign familial neonatal convulsions syndrome (BFNC) (Singh, Charlier et al. 1998). Mutations in the voltage-gated potassium channel $\mathrm{K}_{\mathrm{v}} 1.1$ has been found to be associated with partial epilepsy (Zuberi, Eunson et al. 1999). Deletion of this channel in mice has shown to cause epilepsy (Smart, Lopantsev et al. 1998). Mutations in the voltage-gated calcium channel $\mathrm{Ca}_{\mathrm{v}} 3.2$ have been found in patients with childhood absence epilepsy. These mutations alter channel gating, surface expression, 
and activate gene transcription cascades to induce hyper-excitable neurons (Eckle, Shcheglovitov et al., in press). Repeated seizures lead to changes in the neuronal network, leading to the occurrence of spontaneous seizures. This is referred to as epileptogenesis (Anderson P 2007).

TLE is the most common form of adult focal refractory epilepsy (Leppik, Dreifuss et al. 1987; Spencer, Williamson et al. 1987; Buckmaster, Zhang et al. 2002). Patients with temporal lobe epilepsy have partial complex seizures, which mainly originate in mesial temporal structures of the limbic system like the hippocampus, amygdala or thalamus (Hellier, Patrylo et al. 1998). The partial complex seizures lead to "secondary generalized tonic-clonic seizures" (Hellier, Patrylo et al. 1998). One of the causes of TLE is an initial cerebral injury caused by status epilepticus, trauma, neonatal hypoxia or head injuries in early life (French, Williamson et al. 1993);(Anderson P 2007). These initial injuries lead to a latent period after which the patients develop recurrent seizures (Hellier, Patrylo et al. 1998). During this latent period, the hippocampus is predisposed to sclerosis due to the past injuries (Anderson P 2007) . The hippocampus and cerebral cortex are more prone to seizures because of the presence of recurrent excitatory connections, intrinsically burst generating neurons, "ephaptic interactions among closely spaced neurons" and synaptic plasticity (McCormick and Contreras 2001). A study of 1023 TLE patients has revealed that the main complications suffered by them are cognitive problems like memory, concentration and clear thinking (Bell, Lin et al. 2011).

Status epilepticus (SE) is a serious neurological disorder which requires immediate medical attention. The International League Against Epilepsy (ILAE) defined status epilepticus (SE) as "a seizure that persists for a sufficient length of time or is repeated 
frequently enough that recovery between attacks does not occur" (Lowenstein and Alldredge 1998). The most recent definition of SE is "continuous electrographic seizure lasting more than 5 minutes" (Brophy, Bell et al. 2012). The time point of 5 minutes was chosen because general clinical and electrographic seizures do not persist beyond this time point. If they go beyond this time point, then they aren't likely to stop by themselves (Brophy, Bell et al. 2012). The occurrence of SE increases the risk of those patients to have SE in future and chronic epilepsy (Shorvon 2006). The possibility of developing chronic epilepsy following an incident of SE is three times higher than after an incident of a single seizure (Rossetti and Lowenstein 2011).

The prevalence of SE in a population-based study done in Richmond, Virginia was found to be 41 out of 100,000 people each year (DeLorenzo, Hauser et al. 1996). There are around 100,000 cases of SE each year in United States resulting in 22,000 deaths (DeLorenzo, Hauser et al. 1996). Mortality is high among older patients aged more than 65 years compared to younger patients. Almost $50 \%$ of the patients above 65 years of age die within 30 days after status episode. In younger patients mortality is very rare but they suffer from hippocampal injury (Joshi and Kapur 2012). In US, the cost of SE is approximately $\$ 4$ billion annually (Penberthy, Towne et al. 2005).

In SE the mechanism which acts to stop an individual seizure fails. As a result the seizure persists for a considerable length of time. This inability to abort seizures may be caused from abnormal over excitation or unavailability of sufficient inhibition to stop the seizures. The mechanisms causing SE can be studied from different medical cases. SE was reported from some people who ate mussels contaminated with domoic acid (Perl, Bédard et al. 1990; Teitelbaum, Zatorre et al. 1990). Domoic acid is a glutamate agonist. 
This case scenario and its observations suggested that excitatory amino acids might serve as one of the causes in SE. Inhibiting GABA mediated inhibition also acts as a cause for SE. Compounds like penicillin which antagonize GABA-mediated inhibition cause SE (Lothman, Bertram III et al. 1991).

$\mathrm{SE}$ is classified further into three classes: convulsive SE, non-convulsive $\mathrm{SE}$ and refractory convulsive SE (RSE). Convulsive SE is characterized by generalized tonicclonic seizures and mental status impairment. Non-convulsive SE is characterized by subtle status with no motor component. Pharmaco-resistant convulsive SE is referred to as refractory SE (RSE). 23-43\% of people suffering from SE develops resistance to treatment and becomes refractory (Goodkin and Kapur 2009).

The causes of SE include noncompliance with anti-epileptic drugs, alcohol-related status epilepticus, trauma, tumor, and toxicity from drugs (Lowenstein 1999). Encephalitis, stroke and progressing primary brain tumors are the main causes of RSE (Rossetti and Lowenstein 2011). SE patients should be treated early otherwise the treatment becomes less efficient. In animal studies it has been reported that SE can be treated with benzodiazepines or phenytoin only if administered early (Rossetti and Lowenstein 2011). If not treated, convulsive SE early may lead to various complications like fever, cardiac arrhythmias, electrolyte and glucose imbalance, rhabdomyolysis and respiratory problems (Rossetti and Lowenstein 2011). Studies from animal models have shown that 30 minutes of continuous seizures may lead to lasting neuronal damage (Meldrum and Horton 1973) and pharmacoresistance (Kapur and Macdonald 1997; Mazarati, Baldwin et al. 1998; Brophy, Bell et al. 2012). 


\section{Available treatments for to stop seizures in TLE and SE}

Almost one third of the patients suffering from TLE are unresponsive to anti-epileptic drugs (Riban, Fitzsimons et al. 2009). As a result surgical removal of the epileptogenic focus in the brain is the option left for these patients to have a seizure-free life. The mesial temporal lobe has been seen to be the most common site of seizure origination in most patients (Adil Harroud 2012). However, surgery fails in 20-30\% of the patients (Renate M. Kalnins1 2004; Adil Harroud 2012). Some of the reasons for the failure of surgery have been studied. The temporal structures harboring the epileptogenic zone may not be removed completely during surgery because of various limitations. As a result, the remaining epileptogenic zone keeps causing seizures (Adil Harroud 2012). In some cases, the patient is subjected to a second surgery to completely remove the epileptic zone. The success rate has been shown to be 52-60\% after a second surgery (Adil Harroud 2012). In $30 \%$ of surgical specimens of TLE patients who underwent surgery, both sclerosis in the mesial temporal lobe and extrahippocampal lesion like cortical dysplasia (Lévesque, Nakasato et al. 1991). It's difficult to identify this 'dual pathology' and failure in resecting the mesial temporal sclerosis and the lesion does not promise total treatment from seizures in TLE patients ( $\mathrm{Li}$, Cendes et al. 1999). Even after surgical resection of one hippocampus, relapse of seizures can occur in the contralateral side or extra temporal zones, as observed in 15\% of patients (Adil Harroud 2012).

Surgery is not $100 \%$ effective. Studies have shown that patients who undergo left anterior temporal lobe resection suffer from verbal memory loss whereas right temporal lobe resection leads to visual memory loss (Bell, Lin et al. 2011). TLE patients with bilateral temporal lobectomy suffer high memory loss and significant effect on intellect and 
language (Bell, Lin et al. 2011). Bilateral hippocampal resection of epileptic patient Henry Gustav Molaison resulted in serious anterograde amnesia as well as temporally graded retrograde amnesia (Corkin, Amaral et al. 1997). The entire entorhinal cortex, the medial temporal cortex and fifty percent of the intraventricular portion of the hippocampal formation was surgically removed from this patient. Surgical side effects also depend on the degree of sclerosis in different patients and the seizure onset age.

The strategy to treat SE is to stop the seizures as soon as possible. Benzodiazepines, specifically diazepam, is the most widely used drug to treat SE (Goodkin and Kapur 2009). The seizures of $80 \%$ of SE patients were treated successfully with diazepam when administered within 30 minutes of start of seizures (Lowenstein and Alldredge 1993). On the contrary, only $40 \%$ of the patients had seizure suppressant effect of diazepam when the treatment was started after two hours (Lowenstein 1998). The effectiveness of benzodiazepines to stop seizures reduces significantly with time (Kapur and Macdonald 1997). Some drugs like phenytoin or phenobarbital are given to SE patients along with diazepam. This contributes to the "anti-seizure effect" (Lowenstein 1998). SE patients who do not respond to these drugs are treated by injecting anesthetics like barbiturate, propofol, or midazolam intravenously (Lowenstein 1998). Other anticonvulsant drugs that have been used to stop status epilepticus include: topiramate (Stojanova and Rossetti 2012), pregabalin (Novy and Rossetti 2010), levetiracetam (Knake, Gruener et al. 2008) and lacosamide (Kellinghaus, Berning et al. 2011). 


\section{Brain structures involved in temporal lobe epilepsy}

Temporal lobe epilepsy (TLE) has its focus in the limbic structures of the temporal lobe like the hippocampus, entorhinal cortex (EC), amygdala and midline thalamus. Studies have not clearly identified exactly which particular area is responsible for seizure origination. The hippocampus is composed of the unidirectional neuronal "trisynaptic circuit" which allows information to travel from one part to another (Anderson P 2007) (Fig. 1). The superficial layers of the entorhinal cortex (EC) project unidirectionally to the dentate gyrus via the perforant pathway. The layer III neurons of EC project to pyramidal cells of the stratum-moleculare of CA1, CA2 and CA3 via the temporoammonic pathway. This pathway is separated from the perforant pathway and signal via the distal branches of the pyramidal cells of the cornuammonis region (Anderson P 2007). The axons of the granule cells of the dentate gyrus called the mossy fibers project to the pyramidal cells of the CA3 region (Anderson P 2007). The CA3 pyramidal cells form the major input to the CA1 field of the hippocampus. The CA1 region of the hippocampus closes the loop by projecting to the subiculum and the EC. The subcortical projections of the brain connect to the hippocampus via the subiculum.

The volume of the EC is significantly decreased in patients suffering from TLE (Du, Whetsell Jr et al. 1993; Du, Eid et al. 1995). Studies have shown that stimulating only the EC can evoke spontaneous interictal spikes in the hippocampus (Rutecki, Grossman et al. 1989). The EC layer II neurons become hyper-excitable in animal epilepsy models. Stimulation of the deep layers of the EC shows long excitatory synaptic responses in layer II neurons (Bear, Fountain et al. 1996; Tolner, Kloosterman et al. 2005; Kumar and Buckmaster 2006); (Scharfman 1996). Layer III neuron loss has been found in rat model 
of epilepsy (Du, Eid et al. 1995). Seizure suppression has been successful in TLE patients by surgical removal of hippocampus (Siegel, Wieser et al. 1990). The major excitatory input to the dentate gyrus (DG), CA3 and CA2 regions are formed by the EC neurons via the perforant and temporoammonic paths (Segal and Landis 1974; Steward 1976) (Fig. 1). EC layer II and III also project to CA2 layer of hippocampus (Chevaleyre and Siegelbaum 2010). New studies have revealed that EC layer II and CA2 neurons are reciprocally connected (Rowland, Weible et al. 2013). Hyperactivity in the EC will lead to neuronal excitability in the hippocampus, further exciting the hippocampal-EC circuit due to reciprocal connections (Buckmaster and Dudek 1997; Kobayashi and Buckmaster 2003).

CA3 pyramidal cells also have a tendency to generate seizures due to their intrinsically burst generating properties (McCormick and Contreras 2001). The neurons in this region have the ability to generate intrinsic bursts of 2-5 action potentials at 200-350 Hertz when they were depolarized for a short time (McCormick and Contreras 2001). The dentate granule cells of the dentate gyrus project mossy fibers connecting with the pyramidal cells of the CA3 field of hippocampus. The pyramidal cells of the CA3 project to the CA1 layer through the Schaffer's collateral axons. The mossy fibers from the dentate granule cells sprout in TLE patients and extend to other hippocampal regions forming new synaptic connections (Sutula, Cascino et al. 1989; Buckmaster, Zhang et al. 2002).

These previous studies suggest that the EC and the CA3 region will be desirable targets to inject adeno-associated virus (AAV) to disrupt the epileptic circuit and inhibit the spread of seizures to cortical regions. 


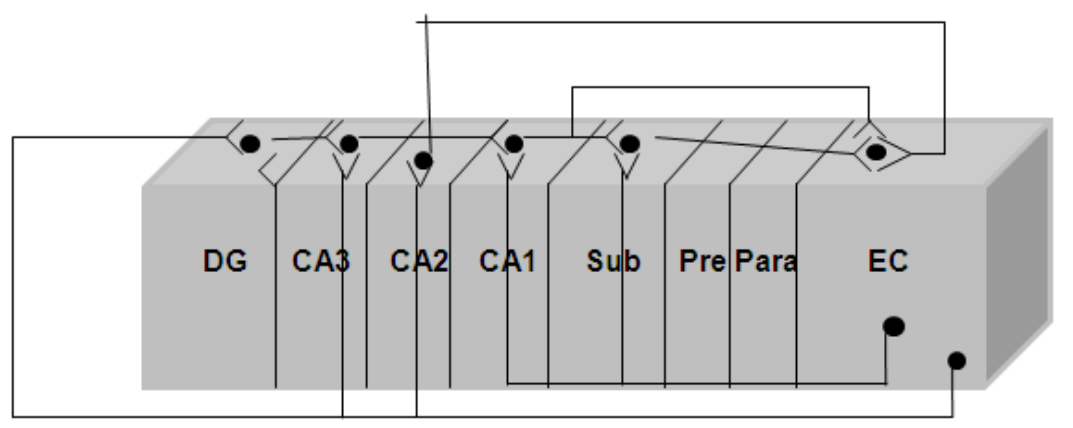

Figure 1: Regions of hippocampal formation \{adapted from (Anderson P 2007)\}

Layer II neurons in entorhinal cortex (EC) project to the dentate gyrus (DG) and CA3 field via the perforant pathway. Layer III neurons project to CA1 and Subiculum (Sub). The neurons projecting from DG to CA3 are the mossy fibers. Neurons projecting from CA3 to CA1 region are Schaffer collaterals. EC layer II neurons project to $\mathrm{CA} 2$ which reciprocally project back to $\mathrm{EC}$. This picture is showing the projections along the transverse axis of the hippocampal formation DG is situated at proximal end while EC is being located at distal end.

\section{TLE pathology}

Hippocampal sclerosis is the most common pathophysiology found in patients with recurrent seizures. Hippocampal sclerosis can be focal (Babb, Brown et al. 1984), unilateral, or in severe cases bilateral (Van Paesschen, Connelly et al. 1997). Loss of CA1 pyramidal cells and hilar cells has been reported (Anderson P 2007). The hilus consists of 
neuropeptide Y (NPY) and somatostatin-rich inhibitory interneurons, which are present in the molecular layer of dentate gyrus. These interneurons are lost as a result of hippocampal sclerosis (Mathern, Babb et al. 1995). NPY rich neurons undergo reorganization after seizures (Vezzani, Schwarzer et al. 1996).

$10 \%$ of dentate granule cell loss and CA2 pyramidal cell loss have been reported suggesting that these neurons are more resistant to an epileptic insult (Bruton 1988). MRI studies have shown significant loss of volume of the EC (Salmenperä, Kälviäinen et al. 2000). However, neuronal loss in EC has been observed in patients where no hippocampal sclerosis can be found ( $\mathrm{Du}$, Whetsell $\mathrm{Jr}$ et al. 1993). Glutamate excitotoxicity is the primary reason for neuronal damage due to seizures. Glutamate receptor activation leads to calcium influx through NMDA and GluR2 lacking AMPA receptors (Lipton and Rosenberg 1994; Tanaka, Grooms et al. 2000). Excess calcium leads to activation of various reaction pathways ultimately resulting in neuronal death. After neuronal loss, synaptic rearrangements will occur.

Mossy fiber sprouting and reorganization is one of the important events that occur in the development of chronic seizures (Sutula, Cascino et al. 1989). Mossy fibers are axons from dentate granule cells of the dentate gyrus. The dentate granule cells acts as a gating brake against seizure spread to the other limbic structures (Lothman and Edward III 1993). The resting membrane potential of these cells is hyperpolarized and they are typically non-excitable due to strong tonic GABAergic inhibition (Anderson P 2007). These cells do not exhibit burst properties like the CA3 pyramidal neurons (Anderson P 2007). Repeated seizures alter the properties of dentate granule cells, including increased mossy fiber sprouting. Increased growth of mossy fibers and reorganization of this mossy 
fiber pathway has been found in chronic epileptic rats as well as in humans (Sutula, Cascino et al. 1989). Extensive mossy fiber sprouting has been seen to innervate other granule cells and CA3 pyramidal neurons in hippocampal sclerotic specimens from humans (Babb, Kupfer et al. 1991). This increased innervation results in hippocampal hyper-excitability.

Seizure induced neurogenesis has also been seen in the pilocarpine rat model of epilepsy (Parent, Timothy et al. 1997). Ectopic granule cells have also been seen in the hilus and inner molecular layer of dentate gyrus in human surgical specimens of TLE patients (Houser 1990).

\section{The use of gene therapy for the treatment of epilepsy}

Epilepsy characterized by focal seizures like temporal lobe epilepsy is a good candidate for gene therapy. Gene therapy promises specific targeting of a region of interest without causing much harm to healthy surrounding tissue as well as fewer side effects compared to surgical resection of hippocampus and anti-epileptic drug administration. Gene therapy combined with stereotactic surgery can be used as an efficient gene delivery route to a specific brain region. Using this technique high level of transgene expression can be seen in various rodent model of epilepsy using adeno-associated virus (AAV) as vectors.

N-methyl D-aspartate receptor 1 (NMDAR1) subunit is essential for the formation of a functional NMDA receptor. Habermann and his colleagues (2002) used the approach of decreasing the NMDA receptor mediated excitation by antisense NMDAR1 receptor cDNA in order to attenuate focal seizure sensitivity. Injecting recombinant adeno associated virus (rAAV) coding NMDAR1 antisense cDNA under the cytomegalovirus 
(CMV) promoter caused significant decrease in seizure susceptibility. The drawback of their study was an increase in seizure susceptibility, observed when tet-off directing NMDAR1 was used for in vivo experiments (Haberman, Criswell et al. 2002).

Raol and his colleagues (2006) targeted the GABA receptor subunits. In a rat model of epilepsy $\alpha 4$ subunit of GABA is up-regulated and $\alpha 1$ subunit is down-regulated. They designed GABRA4 promoter directing the $\alpha 1$ subunit of GABA AAV5 vector and injected in the hippocampus of epileptic rats. A significant reduction in seizures was observed. The drawback of this study was that the gene expression by AAV lasted for only 2 weeks and also mRNA level was decreased after that time (Raol, Lund et al. 2006).

Neuropeptides have emerged as hopeful candidates for gene therapy in temporal lobe epilepsy. Neuropeptide Y (Noe, Pool et al. 2008) and galanin (McCown 2009) have been seen to have modulatory effects on seizures. They have been found to be released during seizures (Riban, Fitzsimons et al. 2009). Galanin suppresses hippocampal seizures by increasing potassium conductance and decreasing calcium conductance (McCown 2009). It inhibits glutamatergic transmission presynaptically and also serves as a neuroprotective agent (Riban, Fitzsimons et al. 2009). McCown and his group injected AAV directing fibronectin secretory sequence fused to galanin in piriform cortex of kindled animals. The latency for seizure was higher and no seizure activity was observed at the site of injection. Although use of human galanin decreased the number of seizures, the latency to seizures was unaffected (McCown 2009). 
In animal models of epilepsy, NPY mRNA and protein was expressed in the dentate gyrus in the hippocampus (Vezzani, Sperk et al. 1999). In vitro studies have shown that application of NPY in hippocampal slices decreases glutamatergic excitation (Riban, Fitzsimons et al. 2009). Injection of AAV1/2 virus directing pre-pro-NPY in hippocampus resulted in delayed onset of seizures and decrease in occurrence of seizures (Richichi, Lin et al. 2004; Riban, Fitzsimons et al. 2009). Studies with AAV expressing different genes of choice have so far not been successful in curing chronic seizures. Therefore, there is a need to find novel targets to alleviate chronic seizures.

\section{Gene of choice- Trek potassium channel}

(TREK-M) which is a mutated form of Trek-1 channel was chosen as the gene to treat seizures in epileptic rats. The hypothesis is that TREK-M incorporation in the hyperexcitable neurons will silence them and treat seizures. A brief introduction about Trek-1 channels and its regulation is discussed below.

\section{Trek-1 potassium channel and its regulation}

Trek-1 potassium channels belong to the family of two pore domain (K2P) potassium channels also known as background or leak channels. These channels play an important role in maintaining the resting membrane potential and regulating neuronal excitability. In 1995 this new family of potassium channels with "two pore domain in tandem" was revealed (Enyedi and Czirjak ; Ketchum, Joiner et al. 1995). K2P channels were further divided into six subfamilies on the basis of their similarity in genetic sequence and function. They are TWIK (Tandem of pore domains in a weak inwardly rectifying potassium channel), TREK (TWIK related potassium channel), TASK (TWIK related 
acid sensitive potassium channel), TALK (TWIK related alkaline $\mathrm{pH}$ activated potassium channel), THIK (Tandem pore domain halothane inhibited potassium channel) and TRESK (TWIK related spinal cord potassium channel) (Enyedi and Czirjak).

Trek- 1 or $\mathrm{K}_{2 \mathrm{P}} 2.1$ is encoded by the $K C N K 2$ gene and belongs to the TREK subfamily of two pore potassium channels. Human Trek-1 expression is found in brain particularly in GABA containing interneurons of the caudate nucleus and putamen (Honore 2007). High expression of Trek-1 is also found in the striatum (Honore 2007), prefrontal cortex, hippocampus, hypothalamus, midbrain serotonergic neurons of the dorsal raphe nucleus and sensory neurons of the dorsal root ganglia (DRG) (Detry, De Roover et al. 2007) (Talley, Solórzano et al. 2001). Apart from the nervous system, Trek-1 channels are also expressed in the gastrointestinal tract (Honore 2007).

A functional Trek-1 channel is composed of two subunits. Each subunit is comprised of four transmembrane domains and two pore loops (Goldstein, Bayliss et al. 2005). Trek-1 plays an important role in maintaining the resting membrane potential and input resistance and as a result, maintains excitability of the neurons.

Trek-1 channels are activated by heat, mechanical stimulation, intracellular acidosis, halothanes and polyunsaturated fatty acids (PUFAs). As a result Trek-1 has a unique gating mechanism compared to the hypothetical background or leak channels.Trek-1 is highly sensitive in the temperature range of $32^{\circ} \mathrm{C}$ to $37^{\circ} \mathrm{C}$. The current amplitude increases by 0.9 fold per degree centigrade rise in temperature (Honore 2007). Trek-1 is activated by membrane stretch and increase in cell volume (Honore, Maingret et al. 2002). The current amplitude of Trek-1 significantly decreases when the external solution 
osmolarity is increased under whole cell configuration (Honore 2007). Trek-1 channels are open when the actin cytoskeleton is disrupted in cell attached patches (Patel, Honore et al. 1998; Honore, Patel et al. 2006; Honore 2007), which means that the actin cytoskeleton acts as a tether to regulate the opening of the Trek-1 channels (Honore 2007). The cytoskeletal composition is changed if Trek-1 is overexpressed and membrane protrusions are observed. This relationship between Trek-1 and actin cytoskeleton plays a role in "neuronal electrogenesis and synaptogenesis"(Honore 2007). The C-terminal domain of Trek-1 plays a considerable role in mechano-gating and gradual deletion of this part makes the channels less affected by stretch and inactivate faster (Honore 2007).

Trek-1 is converted from a mechano-gated to a constitutive leak channel by a decrease in the internal $\mathrm{pH}$ of the cell (Honore 2007). The presence of glutamate (E306) at the proximal $\mathrm{C}$ terminal domain of the channel acts as a proton sensor (Honore, Maingret et al. 2002). Mutation of this glutamate residue locks the channel in an open state and has maximal current activation. This residue acts as a proton sensor and protonation leads to conformational change of the channel which makes the channel prone to stretch regulation (Honore, Maingret et al. 2002).

Trek-1 is activated by halothanes and other inhalation anesthetics (Honore, Maingret et al. 2002). Polyunsaturated fatty acids (PUFAs) like arachidonic acid also activate Trek-1 channels (Patel, Honore et al. 1998; Honore 2007). Activation of the channel by arachidonic acid and halothanes has a neuroprotective action during ischemia (Bayliss and Barrett 2008). Studies in Trek-1 -/- mice have shown that they are prone to ischemia and epilepsy compared to wild type mice (Honore 2007). In wild type mice intravenous or intracerebroventricular injection of linolenate and lysophospholipids provides 
neuroprotection against ischemia and kainic acid seizures (Honore 2007). But in the case of knockout mice, such neuroprotection is absent suggesting the activation of Trek-1 in these specific functions. Arachidonic acid is released during brain ischemia which results in decrease in $\mathrm{pH}$ and swelling of neurons. Decrease of $\mathrm{pH}$, as discussed earlier, causes the Trek-1 channel to open, hence the cell becomes hyperpolarized. Expression of Trek-1 is found in both pre and post synaptic neurons. Hyperpolarization leads to closure of voltage-gated $\mathrm{Ca}^{2+}$ channels. As a result, the intracellular calcium level falls and excitotoxic glutamate release is lowered, too. Post-synaptically, hyperpolarization promotes $\mathrm{Mg}^{++}$blockade of the NMDA receptors. As a result, neurotransmission and glutamate excitotoxicity is reduced (Honore 2007). The AMPA receptor activation is also blocked by the hyperpolarizing effect which cannot remove the $\mathrm{Mg}^{++}$blockade of the NMDA receptors.

Trek-1 is inhibited by the phosphorylation of protein kinase A (PKA) and protein kinase $\mathrm{C}$ (PKC) pathways at specific sites in the C terminal domain. Serine 300 and 333 (S300, S333) amino acid residue sites at the $\mathrm{C}$ terminus region of the channel have been identified to be important for inhibition of the channel via protein kinase A-mediated phosphorylation (Murbartian, Lei et al. 2005). S333 mutation to alanine renders the channels no longer regulated by PKC-mediated pathway, resulting in increased basal current density (Murbartian, Lei et al. 2005). The mutation of S333 to aspartate mimicked phosphorylation and a decreased basal current density was observed, suggesting that this residue is important in channel inhibition via PKC-mediated phosphorylation. Phosphorylation of S333 allows sequential phosphorylation of S300 residue, which also plays an important role in channel inhibition via PKA-mediated pathway. 
The triple mutant TREK-M channel is no longer regulated by protein kinase A and protein kinase $\mathrm{C}$ modulation. As a result this will be constitutively active all the time and is no longer regulated by internal phosphorylation by PKA and PKC mediated phosphorylation pathways (Appendix fig.1).

\section{Adeno-associated virus (AAV)}

Gene therapy requires a delivery system to deliver a chosen gene. Delivery system can be of two types: viral or non viral. Lentivirus (LV), Adenovirus (AdV), Retrovirus (RV), Adeno associated virus (AAV) and herpes simplex virus (HSV) are viral delivery systems (Gould and Favorov 2003);(Lu 2004). Naked DNA delivery or polyethylene glycol (PEG) assisted delivery fall under the category of non-viral delivery of gene (Lu, Pereverzev et al. 2004).

Adeno-associated virus (AAV) belongs to the genus of dependoviruses (Carter 2004). AAV is a small, non-enveloped, single stranded DNA virus. The single-stranded DNA (ssDNA) is encapsulated by $20 \mathrm{~nm}$ capsid which is formed from three structural proteins. They are VP1, VP2 and VP3 and are present in a 1:1:10 ratio. AAV has a capacity to package $4.7 \mathrm{~kb}$ single-stranded DNA. The genome has two open reading frames (ORF) called rep and cap. The ORF are flanked by inverted terminal repeats (ITR) which are 145 bp in size. These GC rich palindromic ITRs enclose the replication origin and packaging signals (Lu, Pereverzev et al. 2004). They also play roles in chromosome incorporation. The rep genes are important for gene expression, chromosomal integration, and DNA replication. The cap genes code for the capsid proteins (Heilbronn and Weger). 
Recombinant adeno-associated vector (rAAV) is an ideal vector for gene therapy for the following reasons. It is not toxic and causes little or no side effects; capsid has very low immunogenicity (Riban, Fitzsimons et al. 2009). Serological studies have suggested that these vectors are incapable of causing disease. These vectors are very stable physically and chemically and can stay for a long time at temperatures like 60 degrees $\mathrm{C}$ (Heilbronn and Weger). Long-term gene expression of these vectors has been found in brain, liver and muscles of animal models which remained stable for months and years (Heilbronn and Weger). The discovery of different serotypes of rAAV with different tropism for distinct neuronal types and brain has made it easier for gene therapy by rAAV (Riban, Fitzsimons et al. 2009). Recombinant AAV is mostly neuronal specific and does not transduce glial cells (Riban, Fitzsimons et al. 2009). Serotypes like 1, 5, 7 and 8 have shown high transgene expression in brain, sprawling several millimeters (Burger, Gorbatyuk et al. 2004; Riban, Fitzsimons et al. 2009). Studies done in rat models of temporal lobe epilepsy have shown that serotype 5 have transduction efficiency significantly higher than serotype (Weinberg, Blake et al.).

The only disadvantages of using rAAV are its short packaging capacity of $4.7 \mathrm{~kb}$ and laborious vector production (Heilbronn and Weger).

Recombinant adeno associated virus (rAAV) is a single-stranded DNA virus. The conversion of single stranded DNA into double stranded DNA has been suggested as one of the rate-limiting steps for gene expression (McCarty 2008). The vector genome becomes unstable for a brief period of time during the conversion of single-stranded DNA into double stranded one. This leads to a considerable loss of gene expression (Wang, Xie et al. 2007; McCarty 2008). As a result the overall vector efficiency is 
affected. Self-complementary AAV (sc AAV) vectors have both plus and minus DNA strands packaged as a single molecule. As a result, scAAV does not require undergoing the rate limiting step of conversion to double stranded DNA, and expression is comparatively higher than rAAV vectors (McCarty 2008).

Many groups have suggested using AAV as a possible treatment for epilepsy (Riban, Fitzsimons et al. 2009). The use of rAAV has earned preference for viral-delivered gene delivery (Carter 2005). Also, AAV developed by Neurologix (NLX-P101) has passed Phase 2 clinical trial for Parkinson's disease. The use of rAAV has certain advantages. The target DNA delivered by rAAV does not integrate into the host genome and expresses by episome formation (Penaud-Budloo, Le Guiner et al. 2008). As a result, disruption of endogenous gene transcription is very minimal. Little or no inflammatory response is produced by rAAV on being introduced into the human body since most have prior rAAV exposure. In the case of rAAV the gene expression efficiency is low (McCarty 2008)). The gene expression efficiency is significantly higher in the case of scAAV (self-complementary AAV) particles (McCarty 2008). The scAAV's low packaging capacity of 2500 bp prohibits its use for doxycycline regulated cassette.

Adeno-associated virus is an excellent choice of gene delivery in the brain of these epileptic rats. Due to high transduction rate and neuronal selectivity, we have chosen to use AAV5 serotype to package the TREK-M channel and deliver in the hippocampus of rat models of temporal lobe epilepsy. We used a self-complementary (sc) AAV since it infects 10-times more neurons than the commonly used single-stranded AAV (McCarty 2008). 


\section{Development of a Dox-On targeting vector}

Due to safety concerns, it would be a good idea to develop an AAV targeting vector that is regulated by a drug. This drug regulation would enable doctors to titrate the dose and also would permit termination of the therapy if there are any limiting side effects.

One of the extensively used gene expression regulatory systems in eukaryotes is the tetracycline regulatory system. This system offers many advantages like "strict on/off regulation, high inducibility, short response times, specificity, no interference with cellular pathway, bioavailability of a non toxic inducer, and dose dependence" (Mizuguchi and Hayakawa 2002). It is based on the E. coli Tn10 resistance operon (Wissmann, Meier et al. 1986). A tet repressor protein (Yang, Tetreault et al.) and Tet operator DNA sequence (TetO) constitutes the operon (Stieger, Belbellaa et al. 2009). The tet regulatory system operates on the following principle: TetR binds to TetO in absence of antibiotic tetracycline or its derivative doxycycline. In the presence of the antibiotic, TetR undergoes a conformational change and no longer remains bound to TetO (Stieger, Belbellaa et al. 2009). Tet regulated systems originally used two expression cassette systems. One cassette contained the regulatory protein (e.g. rtTA) under a promoter and the second cassette consisted of the tetracycline responsive element (TRE) situated 5' of the gene of interest (Nishijima, Yasunari et al. 2009). When four amino acids of the tetR were mutated, it's response to tetracycline reversed to activation

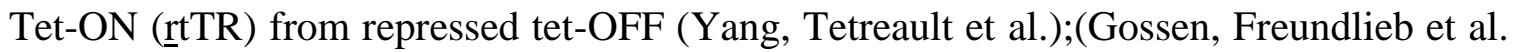
1995). Doxycycline (Dox) is a tetracycline analog and it activates the rtTR (Dox-ON). In the most recently developed tet regulatory system, the tet repressor protein is coupled to a small segment of herpes simplex virus (VP16) protein (Yang, Tetreault et al.). This tetR- 
VP16 fusion acts as a strong transcriptional activator (Manfredsson, Burger et al. 2009). This transactivator then binds to tetO and initiates transcription of the gene of choice in the presence or absence of tetracycline.

An AAV Dox-OFF single expression cassette has been developed which has produced tight and long-term expression of glial cell line derived neurotrophic factor (GDNF) in the substantia nigra (Manfredsson, Burger et al. 2009). Using a similar approach, we have developed a Dox-ON targeting vector expressing TREK-M and mCherry which can be turned on by doxycycline administration.

Doxycycline and has been widely used in clinics for almost 30 years as an antibiotic. It is approved by FDA for human use, has a serum half life of 14-22 hours and penetrates the brain (Stieger, Belbellaa et al. 2009). It is also used for infectious diseases such as Lyme disease, malaria and multi-resistant Staphylococcus aureus infections. The side effects of long-term use of Dox include renal toxicity and dose dependent photosensitivity (Stieger, Belbellaa et al. 2009). It should be noted that the latest generation of tet repressors have been optimized to respond to dox, requiring 100-fold lower concentrations than used for bacterial infections.

\section{GABRA4 promoter}

The packaging limit of a recombinant AAV vector is $4.7 \mathrm{~kb}$. In order to package all our chosen genes within that size limit, promoters are required which are both small and neuronal-specific. GABRA4 is the human promoter of $\mathrm{GABA}_{\mathrm{A}} \alpha 4$ receptors (Sun, Sieghart et al. 2004). 
Arguably, the most important inhibitory neurotransmitter in central nervous system (CNS) is GABA (McDonald, Pelzer et al. 1994). The binding of GABA with GABA receptors results in fast inhibitory action (Raol, Lund et al. 2006). $\mathrm{GABA}_{\mathrm{A}}$ receptors are fast activating $\mathrm{Cl}^{-}$channels. These channels are activated by binding of GABA with the receptors and results in influx of $\mathrm{Cl}^{-}$ions into the neuron resulting in hyperpolarization of the membrane thus causing resultant inhibitory action (Macdonald and Kelly 1994). $\mathrm{GABA}_{\mathrm{A}}$ receptors are composed of multiple subunits like $\alpha 1-\alpha 6, \beta 1-\beta 4, \gamma 1-\gamma 3, \delta, \varepsilon, \varphi, \pi$ (Macdonald and Kelly 1994; YogendraSinh H Raol 2006).

The dentate granule cells in the hippocampus express $\mathrm{GABA}_{\mathrm{A}} \alpha 4$ receptors (Sun, Sieghart et al. 2004). The expression of $\mathrm{GABA}_{\mathrm{A}} \alpha 4$ receptors have been found to increase in the dentate gyrus of rat model of temporal lobe epilepsy as well as in TLE humans (BrooksKayal, Shumate et al. 1998). This upregulation involves the transcription factor Egr-3, which is also found to have increased expression in epileptic rodents. Previous studies have shown that expression of yellow fluorescent protein from this promoter is $30 \%$ higher in pilocarpine-induced epileptic rodent dentate gyrus. This upregulation is mediated by a transcription factor 3 (Egr-3). This transcription factor has also been found to be upregulated in epileptic rats. In human focal epilepsies the transcription factors Egr1, Egr-2 and c-fos have been found to be upregulated (Rakhade, Yao et al. 2005). As a result of these findings, the GABRA4 promoter has emerged as our choice of promoter to deliver TREK-M in epileptic rats. In our lab we have recloned this promoter and have modified the 3' end by adding CMV minimum promoter. The CMV minimum sequence is a TATA box and TFIIB binding site(Loew, Heinz et al.). We have found that it enhances the promoter activity by 2 fold (see Fig. 2). Studies have also shown that in 
human focal epilepsy there is an increased expression of transcription factors Egr-1, Egr2 and c-fos (Rakhade, Yao et al. 2005). Human and rat GABRA4 have consensus binding sites for the Egr family (Rakhade, Yao et al. 2005). Also we have used 542 bp fragment of GABRA4 promoter which is easier to package in an AAV packaging vector with size limit of $2.4 \mathrm{~kb}$ only.

\section{Pilocarpine model of epilepsy}

Pilocarpine is the chemical convulsant which has been used to induce epilepsy in our lab experiments and is discussed further below.

W.A. Turski in 1983 reported that seizures can be produced in rodents by the administration of cholinergic agents (Turski 2000). This model has been accepted as a popular model of epilepsy since that time. The main features of temporal lobe epilepsy are presence of epileptic focus in the limbic system, early cerebral insult, latent period and hippocampal sclerosis which leads to network reorganization (Curia, Longo et al. 2008). These features are easily reproduced in the pilocarpine model of epilepsy in rodents. In the pilocarpine model of epilepsy, acute status epilepticus is induced by intraperitoneal injection of pilocarpine. This acute state is followed by a latent period, which further leads to spontaneous recurrent seizures development (Curia, Longo et al. 2008). Pilocarpine is a muscarinic agonist which activates M1 muscarinic receptors directly. Studies on M1 receptor knockout mice have shown that pilocarpine is unable to produce seizures in them (Hamilton, Loose et al. 1997; Curia, Longo et al. 2008). Status epilepticus is generated as a result of imbalance between excitatory and inhibitory neurotransmission created by pilocarpine. Also, an increase in glutamate levels in 
hippocampus has been reported in in vivo microdialysis studies due to administration of pilocarpine (Curia, Longo et al. 2008). NMDA receptors are also activated by this drug which aids in the maintenance of seizures (Curia, Longo et al. 2008). Pilocarpine by itself can induce seizures at a high dose ranging between $350-400 \mathrm{mg} / \mathrm{kg}$ in rodents (Turski 2000). Injecting lithium in the rodents prior to pilocarpine administration potentiates the effect of pilocarpine in generating seizure at higher doses. Lithium $(3 \mathrm{mEq} / \mathrm{kg})$ is injected in the rats 24 hours prior to pilocarpine injection. No seizures are produced if the interval of injecting lithium and pilocarpine is more than 48 hours (Honchar, Olney et al. 1983). Injecting lithium results in the requirement of much lower dose of pilocarpine $(30 \mathrm{mg} / \mathrm{kg})$ for seizure generation (Honchar, Olney et al. 1983; Curia, Longo et al. 2008). The behavioral features of the rodents are characterized by "salivation, piloerection, tremor, chromodacryorrhea (bloody tears) and diarrhea" after five minutes of pilocarpine injection (Honchar, Olney et al. 1983). These continue for 20 minutes and then motor seizures start including sniffing, chewing, head bobbing and blinking. Sometimes "wetdog shakes" can be seen. These persist for another 10 minutes after which the animals exhibit "continuous myoclonic jerks of head and forelimbs" (Honchar, Olney et al. 1983). This phase is called status epilepticus and can continue for six hours. In our protocol, this phase is ceased by the application of diazepam after 2 hours of application of pilocarpine to decrease mortality rate of rodents.

\section{Specific Aims}

Epilepsy is a neurological disorder affecting millions of people globally. Temporal lobe epilepsy (TLE) is the most common form of adult epilepsy. A significant number of SE and TLE patients are unresponsive to anti-epileptic drugs. The most common and 
effective treatment for these patients are surgical resection of the affected temporal lobe or hippocampus. Surgery is not fully effective and leaves patients with major side effects. As a result, a novel treatment for SE and chronic TLE is a necessity. Seizure foci of TLE mainly lie in the hippocampus and also in the entorhinal cortex, amygdala and midline thalamus. Studies have shown that entorhinal cortex and cornuammonis 3 part of the limbic system play a significant role in TLE. Gene therapy has evolved as a good treatment option for these kinds of epilepsy. The goal of my thesis was to find an alternative adeno associated virus mediated gene therapy treatment for status epilepticus and temporal lobe epilepsy.

In my thesis work I tested the hypothesis that adeno associated virus (AAV) mediated delivery of mutated potassium leak channel (TREK-M) can effectively silence hyperexcitable neurons and control seizures. Based on this hypothesis, I had three broad specific aims. My first specific aim was to validate tools before advancing to in vivo studies. Under my first specific aim, I had two sub aims. They are as follows:

Subaim 1: Validation of neuron specific promoters to drive our gene of choice for in vivo experiments

Subaim 2: Validation of brain regions to inject our gene via adeno-associated vector.

Once the validation of the correct promoter to drive our chosen gene and the particular brain region that would be ideal to suppress seizures was done, it was time to move forward to in vivo studies. My second and third specific aim was focused on in vivo studies. The second aim of this study was to investigate the effect of scAAV5-GABRA4TREK-M on acute seizures induced by Lithium/Pilocarpine in rats. I asked specifically 
three questions in order to answer my specific aim 2. Those three questions were three sub aims which are as follows.

Sub aim 1: Whether scAAV5-GABRA4-TREK-M has any effect on behavioral stage score (BSS)?

Sub aim 2: What effect scAAV5-GABRA4-TREK-M has on electrographic scores?

Sub aim 3: What effect scAAV5-GABRA4-TREK-M has on neuronal death?

The experiments, results and discussions of this specific aim is detailed in the chapter 2 of this thesis.

The third specific aim was to investigate the effect of doxycycline regulated expression of TREK-M on chronic epileptic rats. The third specific aim had two sub aims which are as follows:

Sub aim 1: Validating the doxycycline regulated control of TREK-M expression in rats

Sub aim 2: Study the effect of doxycycline regulated TREK-M expression on chronic epileptic rats.

Chapter 4 of this thesis mainly includes the overall discussions of my study and future aims of this project. 


\section{CHAPTER 2: A potassium leak channel silences hyperactive neurons and ameliorates status epilepticus}

\section{ABSTRACT}

The focus of this study was to investigate the effect of gene therapy treatment for acute seizures induced by pilocarpine in a rat model of epilepsy. Seizures are broadly classified as generalized, partial, or focal. Continuous seizures lasting for more than 5 minutes is referred as status epilepticus which is a serious medical emergency (Brophy, Bell et al. 2012). Almost 4 in 1000 people suffer from status epilepticus. 23-40\% of SE patients become refractory to anti epileptic drug. The occurrence is higher in people aged less than one year and more than 60 years (Hesdorffer, Logroscino et al. 1998). Gene therapy mediated by adeno-associated viral (AAV) vectors has been in development for various neurodegenerative diseases like Alzheimer's and Parkinson's disease. In this study we have investigated the effect of adeno-associated virus-mediated expression of a constitutively-active potassium $\mathrm{K}+$ leak channel using Trek-1(TREK-M) on acute seizures induced by intraperitoneal injection of pilocarpine. Our results demonstrate that the expression of TREK-M in rodent brain prior to pilocarpine insult resulted in significant reduction of SE. The total seizure duration of TREK-M pre-injected rats was also significantly less. TREK-M injection also caused significant reduction in neuronal death around the injected areas like the entorhinal cortex, CA3 and CA1 regions of the brain. These findings demonstrate that TREK-M can silence hyper-excitable neurons in the brain of epileptic rats and treat acute seizures. This study paves the way for an alternative gene therapy treatment of patients suffering medically refractory focal 
seizures and provides the rationale for studies of AAV-TREK-M's effect on spontaneous seizures in chronic models of temporal lobe epilepsy.

\section{MATERIALS AND METHODS}

\subsection{Transfection}

HEK-293 and ND7/23 (rat dorsal root ganglion neuron X mouse neuroblastoma; Sigma) were maintained in DMEM-F12 supplemented with 10\% FBS and $1 \%$ penicillin/streptomycin under standard tissue culture conditions $\left(5 \% \mathrm{CO}_{2}, 37^{\circ} \mathrm{C}\right)$. Cells were grown in $35 \mathrm{~mm}$ Petri dishes. Cells were transfected with Lipofectamine method with $1 \mu \mathrm{g}$ of plasmid DNA. After transfection, cells were maintained in a $95 \% \mathrm{O}_{2} / 5 \%$ $\mathrm{CO}_{2}$ atmosphere at $37^{\circ} \mathrm{C}$ and used for fluorescence experiments between 24-36 hours.

\subsection{Cloning of TREK-M and viral constructs}

Mutant of mouse Trek-1 (TREK-M) was made by the combination of the mutations S333A, S300A and E306A. S333A and S300A remove phosphorylation sites, whereas E306A removes $\mathrm{pH}$ and arachidonic acid sensitivity. By single overlap extension method, the S300A and E306A mutations were introduced to S333A mutant (Horton, Ho et al. 1993; Murbartian, Lei et al. 2005). We used a carboxy terminal truncated version of TREK-M (Murbartian, Lei et al. 2005). Also, we designed a 120 bp long polyadenylation sequence based on SV40. We developed a self-complementary targeting plasmid based on the original deletions of the 3 ' internal terminal repeats (ITRs) described by McCarty and coworkers (McCarty, Monahan et al. 2001). Due to the small packaging capacity of $2.4 \mathrm{~kb}$ of scAAV virus addition of fluorescent protein was not 
possible. This plasmid GTAAVE was sent to University of North Carolina (UNC) Vector Core to be packaged into adeno-associated viral serotype 5 particles.

\subsection{Stereotaxic brain injections}

We have used adult male 95-120 day aged Sprague Dawley (Taconic or Charles River) rats. Animals were acclimated for at least 48 hours before any procedure. These were anesthetized with isoflurane (Sigma), given eye lubricant, and placed on a heated pad inside a stereotactic frame (David Kopf Instruments Model 940 with digital display). The rats were subjected to continuous isoflurane (EZ-B800, World Precision Instruments, Inc) for the entire surgery. They were continuously monitored for anesthesia depth for the complete surgery at every 10 minute interval. An incision was made on the skull. All the muscles and underlying tissues were cleared to expose the bony skull for brain injections and headset mounting. We used a mounted stereotaxic drill (Model 1474, David Kopf Instruments). The injection co-ordinates were ( $\mathrm{mm}$ from bregma): entorhinal cortex, lateral 5, posterior -8.3 ; and depth from -6.6 to 5.6; CA3, lateral 4.6, posterior -4.4 , and depth from -6.6 to -5.1 (Paxinos, Watson et al. 1980). These drilled burr holes were the injection site of AAV virus particles. Seven additional burr holes were drilled into the skull. Four of these were used to put bone anchor screws in order to fix the electro encephalogram (EEG) headset on the head, whereas the other three burr holes were used to place two cortical electrodes and one depth electrode. The depth electrode was made from perfluoroalkoxy-insulated silver wire (0.008” diameter, A-M systems) and placed in piriform cortex at the following coordinates ( $\mathrm{mm}$ from bregma): lateral 5 , posterior -0.3 , and depth -9.5 . $3 \mu \mathrm{l}$ of virus (serotype 5 ; titer $10^{\wedge} 12$ particles $/ \mathrm{ml}$ ) was injected into each site with a $10 \mu \mathrm{l}$ Hamilton syringe connected to an infusion pump controlled by a 
microprocessor at a rate of $20 \mathrm{nl} / \mathrm{sec}$. In order to allow diffusion from the site of injection, the injector was left in place for two minutes after virus infusion. After virus injection, the electrodes were placed in the designated burr holes and the headset was fixed to the head by applying dental cranioplast.

The skin was sutured once the cranioplast dried and set. The rat was given an intraperitoneal injection of $0.1 \mathrm{ml}$ ketoprofen for analgesia. Local application of lidocaine was also used around the suture. The rat was allowed to recover on heated pad after surgery and then returned to vivarium once it was full awake. All procedures were approved by IACUC.

\subsection{Pilocarpine injection}

We have chosen to use pilocarpine induced status epilepticus as the model for temporal lobe epilepsy. All drugs were injected intraperitoneally. Rats were injected with lithium $(3 \mathrm{mmol} / \mathrm{kg}$ ) (Sigma). This pre injection of lithium potentiates the seizure inducing action of pilocarpine (Jope, Morrisett et al. 1986). Twenty hours after lithium injection, scopolamine $(1 \mathrm{mg} / \mathrm{kg})$ was injected into the rats which reduces peripheral effects due to pilocarpine and reduces mortality (Lemos and Cavalheiro 1995). Forty minutes later,

pilocarpine $(50 \mathrm{mg} / \mathrm{kg}$ ) (Sigma) was injected. The rats were injected with diazepam $(10 \mathrm{mg} / \mathrm{kg})($ Sigma) after 2 hours of pilocarpine injection in order to stop the convulsions and reduce mortality.

\subsection{Behavioral stage scoring (BSS)}

The behavior of the rats was monitored for two hours post pilocarpine injections and was measured by the Racine scale (Racine 1972). Rats after pilocarpine injections undergoing 
any change of state viz. sleep, rest or awake received behavioral stage score (BSS) 1. They enter BSS stage 2 shortly which was characterized by head bobbing, chewing, salivation. This stage progressed to severe motor seizures which were characterized by unilateral forelimb clonus (BSS 3), bilateral forelimb or hindlimb clonus (BSS 4) and finally to BSS 5 which included bilateral clonus added with rearing and losing complete balance and falling down. Rat which exhibited running fits were given a score of 6 .

\subsection{Electroencephalogram analysis (EEG)}

The rats were monitored with video/ EEG for 48 hours after pilocarpine injections. The EEG started with non-ictal or non-epileptic pattern. This pattern changes to continuous ictal activity characterized by uninterrupted spikes with a frequency more than $1.5 \mathrm{~Hz}$. This is recorded as stage 3. This continuous pattern is interrupted with periodic epileptic discharges (PEDS) which is called stage 4. Our definition of stage 4 is 10 seconds ictal activity admixed with PEDS. The spikes gradually lose their rhythmicity and the baseline between spikes comes back to baseline. This stage is marked 2. The beginning of stage 2 marks the end of status epilepticus. After stage 2 the rats had discrete seizures which were marked in the EEG. Total status time is defined as the amount of time the rat spent in stage 3 and stage 4 . Total seizure time is measured by adding the time a rat spent in status epilepticus and all the discr.eet seizures the rat had. All these stages are shown in Figure 2 

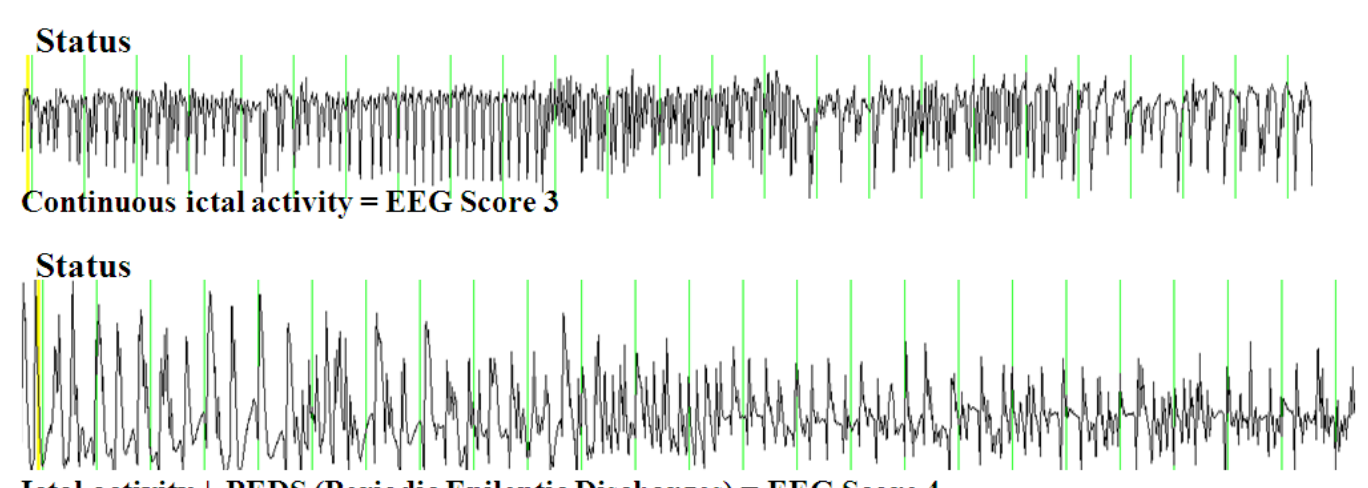

Ictal activity + PEDS (Periodic Epileptic Discharges) = EEG Score 4

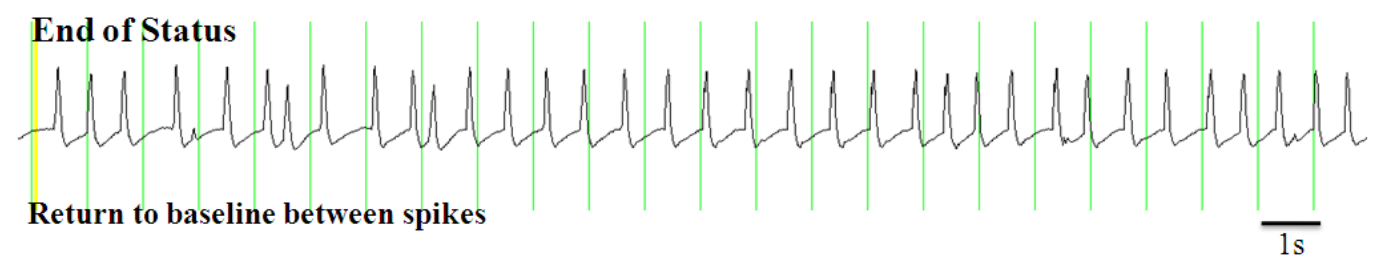

Figure 2: EEG traces showing the different electrographic stages obtained from a pilocarpine injected epileptic rat preinjected with AAV-TREK-M virus

Stage 3: Almost 30 minutes after pilocarpine injection, the EEG shows as continuous fast ictal activity and characterized by uninterrupted spikes more than $1.5 \mathrm{~Hz}$

Stage 4: Transition to stage 4 is marked by the appearance of periodic epileptic discharges with 10 seconds of continuous ictal activity

Stage 2: The continuous ictal activity slowly loses its rhythmicity and enters stage 2. Stage 2 is marked when the frequency of firing becomes less than $1.1 \mathrm{~Hz} / \mathrm{sec}$. Stage 2 marks the end of status epilepticus time. 


\subsection{Tissue preparation for Fluoro-Jade C staining}

The rat was deeply anesthetized with $0.5 \mathrm{cc}$ of pentobarbital (Nembutal Sodium solution, Oak Pharmaceuticals) and was perfused through the ascending aorta with $100 \mathrm{ml}$ of saline first to get rid of all blood from the system followed by $400 \mathrm{ml}$ of $4 \%$ freshly prepared paraformaldehyde. Brains were removed and kept in $4 \%$ paraformaldehyde for 24 hours at $4^{\circ} \mathrm{C}$ for post fixation. Brains were sliced the following day. The brains were embedded in agarose and thin slices (30-50 $\mu$ ) were cut using a Leica VT 1000S vibratome. Horizontal brain slices were collected and kept in 12 well plates. Each well had 4-5 slices.

\subsection{Fluoro-Jade C staining}

Rat brain slices were labeled with Fluoro-Jade $\mathrm{C}$ in order to study neurodegeneration. Slices were mounted on gelatin-coated slides (Lab Scientific Inc.). Fluoro-Jade C consists of the vital fluorochrome named disodium 5'/6' carboxyfluorescin (Schmued, Albertson et al. 1997; Schmued, Stowers et al. 2005). The composition of Fluoro-jade B is as follows. Trisodium 5-(6-hydroxy-3oxo-3H-xanthen-9-yl) benzene, 1,2,4, tricarboxylic acid; disodium 2-(6-hydroxy-3-oxo-3H-xanthen-9-yl)-5-(2,4-dihydroxybenzol) terephthalic acid and disodium 2,5-bis(6-hydroxy-3-oxo-3H-xanthen-9-yl) terephthalic acid (Schmued, Stowers et al. 2005). In our experiments we have used Fluoro-Jade-C to stain degenerating neurons. The precise components of Fluoro-Jade $\mathrm{C}$ are still a trade secret but it consists of sulphate esters of the components of Fluoro-jade B (Schmued, Stowers et al. 2005). While selecting the slices, care was taken to select approximately slices from the same depth for each rat brain. It was ensured that the slices were collected 
from the range of brain depth of -4.0 to -9.0 . This is the same range of depth where the virus was injected in the rat brains. They were dried for 30 minutes on a slide warmer at $50^{\circ} \mathrm{C}$. The slides were then incubated for 5 minutes in sodium hydroxide $(\mathrm{NaOH})$ solution. They were then washed in $90 \%$ ethanol solution and in distilled water for 2 minutes each. Following this step, they were incubated for 10 minutes in potassium permanganate $\left(\mathrm{KMnO}_{4}\right)$ solution for 5 minutes and then rinsed with distilled water for 5 minutes. Slices were then stained in Fluoro-Jade C and DAPI solution for 10 minutes. After this treatment, they are rinsed in distilled water and dried on a slide warmer for 5 minutes. The slides were cleared in xylene and then mounted on Dibutyl Phathalate Xylene (DPX).

\subsection{Image acquisition and data analysis}

Sections of Fluoro-Jade labeled brain slices were acquired on an Olympus IX81 microscope. The images were acquired by IPLAB software. 10X images of hilus, entorhinal cortex were shot whereas $25 \mathrm{x}$ images of CA3, CA1 region were shot for better resolution of individual neurons. The intensity of green of all the images shot at 10X was $500 \mathrm{~ms}$ and that shot at $25 \mathrm{X}$ oil was $100 \mathrm{~ms}$.

All data were analyzed by autosegmentation. The Macro set for analyzing the images had the following lists. The scale was set to $10 \mathrm{X}$ or $25 \mathrm{X}$ depending on the images that was analyzed. Autosegmentation criteria were saved as triangle with split distance

contour and area limit of 2 . In some images when this autosegmentation criteria failed, a region of interest (ROI) was drawn and autosegmentation done with midpoint and distance contour of 100 . 


\subsection{Chemicals and drugs}

Lithium, scopolamine, pilocarpine and diazepam were obtained from Sigma. Nembutal was obtained from Oak Pharmaceuticals. Fluoro-Jade C was obtained from HistochemInc.

\subsection{Adeno-associated virus constructs}

We have modified Trek-1 (TREK-M) by combining all three mutations (S300A, S333A and E306A) by single overlap extension method (Horton, Ho et al. 1993). The TREK-M was subcloned into a pEYFP-N1 vector (Clontech, CMV promoter). AAV vector has been designed using the software DNASIS. Different oligonucleotides were designed using the software OLIGO and the convenient restriction sites have been added. By PCR we have made the altered insert and subcloned it into Stratablunt vector. The vector was purified by screening and sequencing the positive colonies. The AAV targeting vector was cut with appropriate enzyme and then the GABRA4 and TREK-M inserts were added. The AAV targeting vector was ligated to these inserts and was grown in a large scale DNA preparation. We received control scAAV5-GFP virus from University of North Carolina Vector Core. The virus construct is shown in figure 3. 


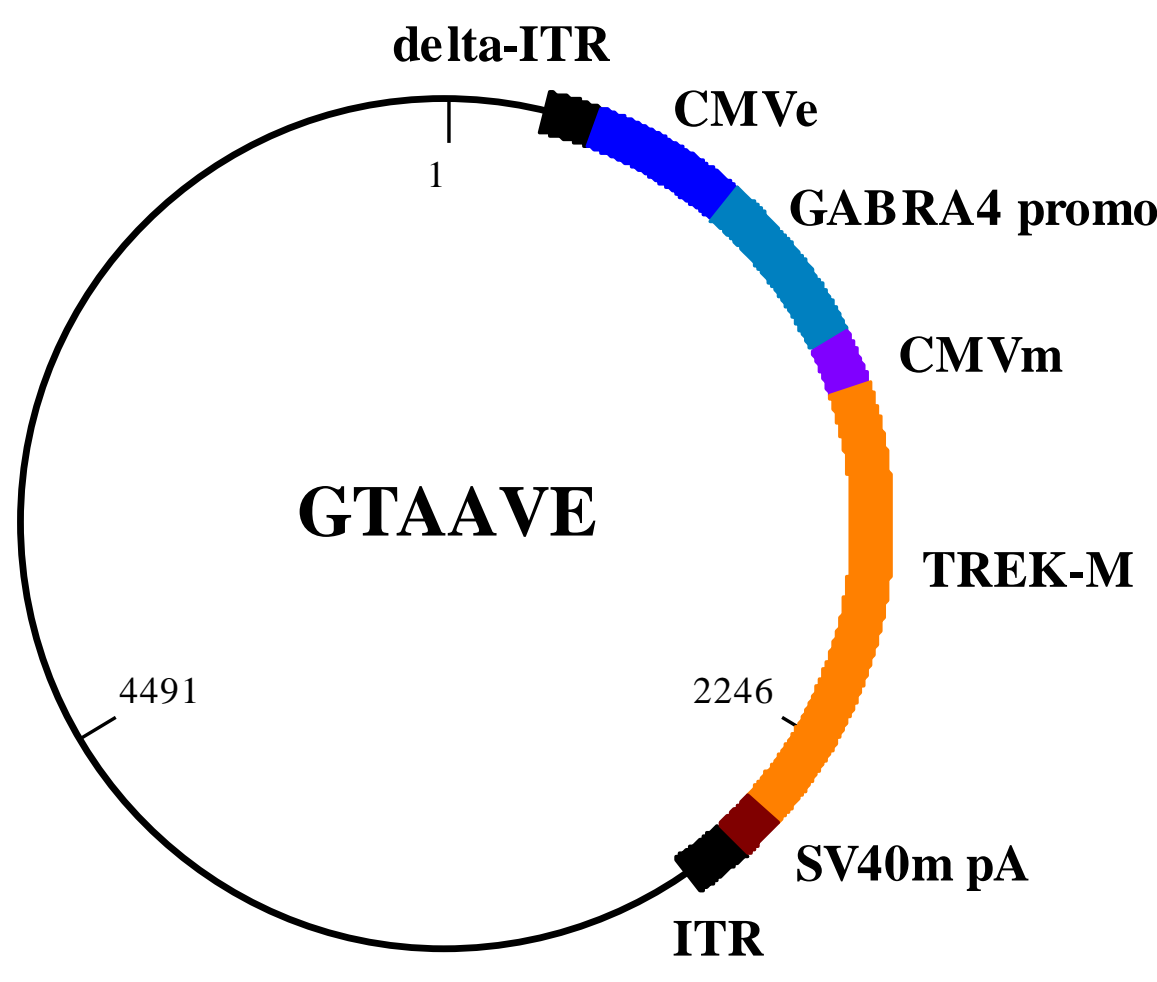

Figure 3. Self-complementary AAV5-GABRA4-TREK-M construct used to see effect of preinjection of this virus in acute seizures in lithium pilocarpine rat model of epilepsy. GABRA4 enhanced by cytomegalovirus enhancer sequence (CMVe) at its 5 ' end and cytomegalovirus minimum sequence (CMVmin) drives the expression of TREK-M. 


\section{RESULTS}

\subsection{The dual enhanced GABRA4 promoter causes robust expression of Venus fluorescent protein}

The GABRA4 promoter by itself is a very weak promoter. No expression of Venus fluorescent protein driven by the GABRA4 promoter was observed in transfected ND9 cells. In order to increase the strength of the GABRA4 promoter we added a CMV enhancer (CMVe) to its 5' end and a CMV minimum (CMVmin) sequence to its 3' end. Three combinations of the promoter were made. The combinations are as follows: GABRA4 promoter enhanced by only the CMVmin sequence at the 3' end (MGY), GABRA4 promoter enhanced by only the CMVe sequence at its 5' end and GABRA4 promoter enhanced both by the CMV enhancer and minimum sequence at 5' and 3' end respectively (EMGY). ND9 cells were transfected with these three enhanced promoter combinations driving Venus fluorescent protein and the original promoter. Fluorescence of Venus fluorescent protein by different combinations of the promoter is represented in Figure 4. Venus fluorescence was 500 times higher in cells transfected with the double enhanced GABRA4 promoter (EMGY) compared to the original promoter (GY). The Venus fluorescence was ten times higher with the MGY construct and fifty times more with the EGY compared to the original promoter. 

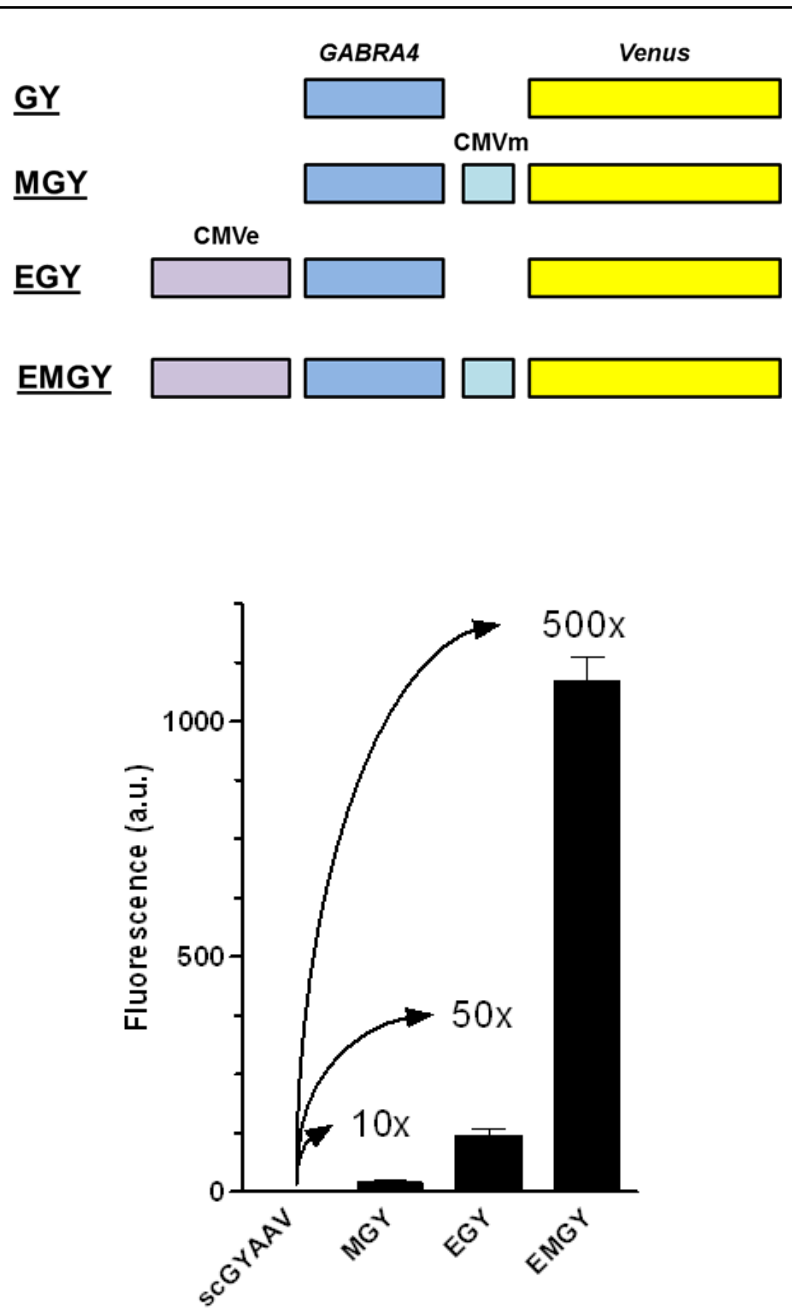

Figure 4: Fluorescent measurement of yellow fluorescent protein Venus driven by various combinations of the GABRA4 promoter.

A: $\mathrm{GY}$ is the original promoter driving mVenus, MGY is the GABRA4 promoter enhanced by CMV minimum sequence at its 3' end, EGY is the promoter enhanced by CMV enhancer sequence at its 5' end and EMGY is the promoter enhanced by both CMV enhancer and minimum sequence at its 5' and 3' end respectively.

B: Fluorescent measurement of different plasmid constructs in ND7/23 neuroblastoma cells. 


\subsection{Dual enhanced GABRA4 promoter retains its neuronal specificity}

It was a concern whether the promoter lost its specificity to neuronal cells after enhancing it by a CMV enhancer and CMV minimum sequence at its 5' and 3' end respectively. In order to test the neuronal specificity of GABRA4 promoter, non neuronal Hek-293 cells and neuronal ND7/23 (rat dorsal root ganglion neuron X mouse neuroblastoma; Sigma), were transfected with the EMGY and GY constructs. We used the CMV promoter driving the expression of yellow fluorescent protein (CMV-YFP) in HEK-293 cells as a positive control. Fluorescence of Venus protein by the original GABRA4 promoter, the enhanced GABRA4 promoter and CMV promoter in HEK-293 and ND7 cells is represented in figure $3 \mathrm{a}$ and $3 \mathrm{~b}$, respectively. Very little expression of yellow fluorescent protein by either the enhanced (EMGY) or the original GABRA4 was observed in HEK-293 cells. CMV-YFP caused robust expression of yellow fluorescent protein in HEK-293 cells. The original GABRA4 construct showed almost no expression. The CMV-GFP showed high expression of green fluorescent protein in ND7 cells. This experiment confirmed that the GABRA4 promoter retained its neuronal specificity even after being modified with enhancer sequences (Fig 5). 

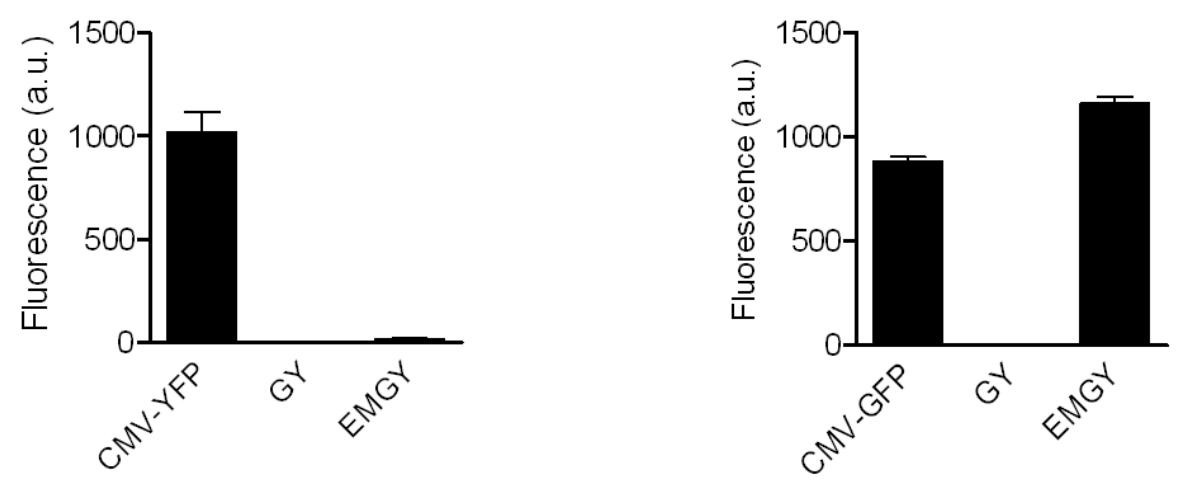

Figure 5: Specificity of GABRA4 promoter in neuronal and non neuronal cells.

A: The plasmids were transfected into ND7/23 neuroblastoma cells and mVenus fluorescence was measured in individual cells. Data were normalized to mVenus expression driven by the CMV promoter. The enhanced GABRA4 directed Venus expression was robust in ND7 cells and almost non-existent in HEK-293 cells. This demonstrates the specificity of GABRA4 as a neuronal promoter was intact even when it was modified.

B: Expression of the same plasmids in HEK 293 cells reveals very low expression from the GY and EMGY versions of the GABRA4 promoter. These results suggest that neuronal selectivity was not affected by addition of the CMV elements. 


\subsection{AAV-GFP injection in CA3 and EC region caused high GFP expression in dentate granule neurons (DGC) and EC layers II, IV and V}

Serotype 5 AAV encapsulated CMV promoter driving green fluorescent protein (AAV5CMV-GFP) viral particles were injected into the $\mathrm{CA} 3$ and $\mathrm{EC}$ regions of naïve rat brain. The goal of this experiment was to validate the injection sites. Representation of the injection sites is shown in figure 4 . We calculated our desired injection site using the rat brain atlas (Paxinos and Watson 1998). The figure depicts the chosen injection site of viral particles in the rat brain. GFP expression was allowed for 4 weeks before preparation of horizontal brain slices and staining with DAPI. Fluorescent imaging of horizontal brain slices revealed bright GFP fluorescence in dentate granule layer, CA3 and EC layers. Robust GFP expression in dentate granule layer, CA3 and EC layers is represented in figure 6a and 6d. We confirmed GFP positive dentate granule neurons by imaging the slice under 100x oil immersion lens as shown in figure 6b. 10x image of CA3 layer showed GFP positive CA3 pyramidal cells as depicted in figure 6c. Injection in EC resulted in GFP positive neurons in layer II, IV and V is shown in figure 6e. 


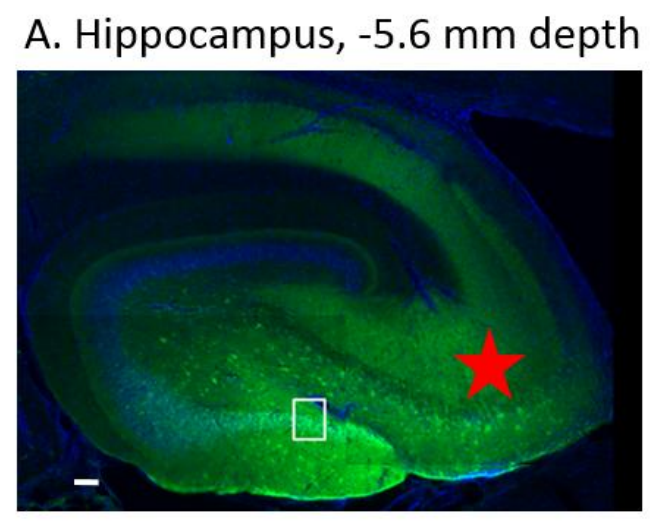

B. DG cell layer

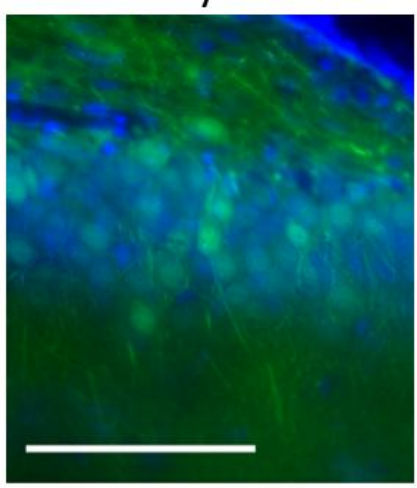

D. Entorhinal cortex



E. EC layer II and IV-V

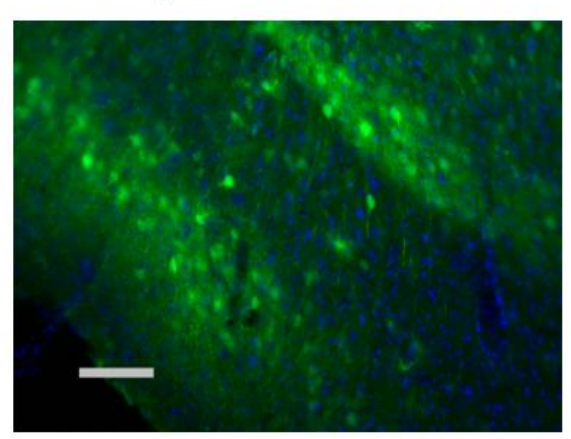

C. CA3 layer

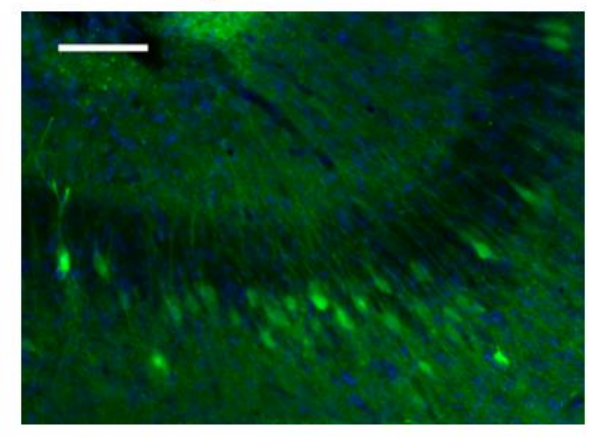

F. EC projections, $-6.8 \mathrm{~mm}$ depth

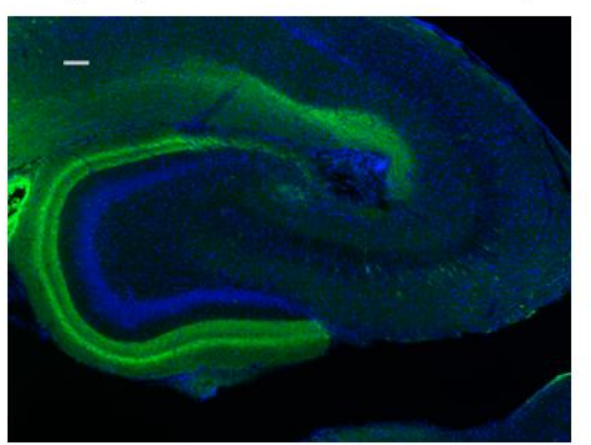


Figure 6: Validation of AAV expression after intraparenchymal injection into the hippocampal CA3 layer and entorhinal cortex (EC). Serotype 5 AAV particles containing GFP driven by the CMV promoter were injected into naïve adult Sprague Dawley rats (red stars show approximate location of injection site). Expression was allowed to proceed for 4 weeks before preparation of horizontal brain slices. Slices were mounted on glass and the nuclei were stained with DAPI. (A) GFP fluorescent images were collected with a 4x objective to show robust expression of GFP in the hippocampus. (B) The boxed area in A was also imaged at 100x to show expression in the dentate granule cell layer. (C) A 10x image of the CA3 layer showing positive GFP labeling in pyramidal neurons. (D) A 10x image showing high GFP expression in the EC and scattered expression in subicular neurons. Due to the 3-dimensional structure of the parahippocampus (van Strien, Cappaert et al. 2009), the dorsal subiculum was often targeted by scAAV-GFP injected into the EC coordinates. (E) The boxed area in $D$ was also imaged at 25x to show expression in EC layers II, IV, and V. (F) A 10x image of the hippocampus showing the perforant path and terminal projections to the DG layer. Scale bars for all images represents $100 \mu \mathrm{m}$. Similar results were obtained in 3 rats. 


\subsection{Viral delivery of TREK-M ameliorates status epilepticus}

We used pilocarpine to induce status epilepticus (SE) since this model has various similarities with human SE (Buckmaster 2004; Curia, Longo et al. 2008; Loscher 2011). Initially SE was defined as seizures lasting thirty minutes or longer (Lothman, Bertram et al. 1989; Lowenstein 1998). However, according to the Neurocritical Care Society the modern definition of status epilepticus has changed to continuous electrographic seizure activity lasting longer than 5 minutes (Brophy, Bell et al. 2012). Adult male rats were injected with CTRL and TREK-M scAAV (serotype 5) particles. Rats were also fitted with a headset. We waited for 4 weeks to allow expression of the virus. Status epilepticus was induced by intraperitoneal injection of pilocarpine as detailed in the methods. We scored both the behavior and electrographic sequelae. The duration of status epilepticus was calculated by adding the total time spent by a rat in electrographic score of three and four. The transition to electrographic score 2 marks the endpoint of status epilepticus. Representative EEG traces are shown in figure 7A. Typical status epilepticus duration of scAAV5-GABRA4-TREK-M pre-injected rat has been represented in Fig 7A and that of control viral particle pre-injected rat have been shown in fig 7B. Representation of status epilepticus EEG in both groups of rats are shown in figure $\mathrm{Ca}$ and $\mathrm{Cb}$. TREK-M significantly reduced the duration of SE by $50 \%$ (P<0.05, Fig 7D). We also measured the total time spent in seizures for the $1^{\text {st }} 24$ hours after pilocarpine injection and found a similar reduction $(\mathrm{P}<0.05)$. However, we found no difference in the onset of $\mathrm{SE}$ and the behavioral score. 

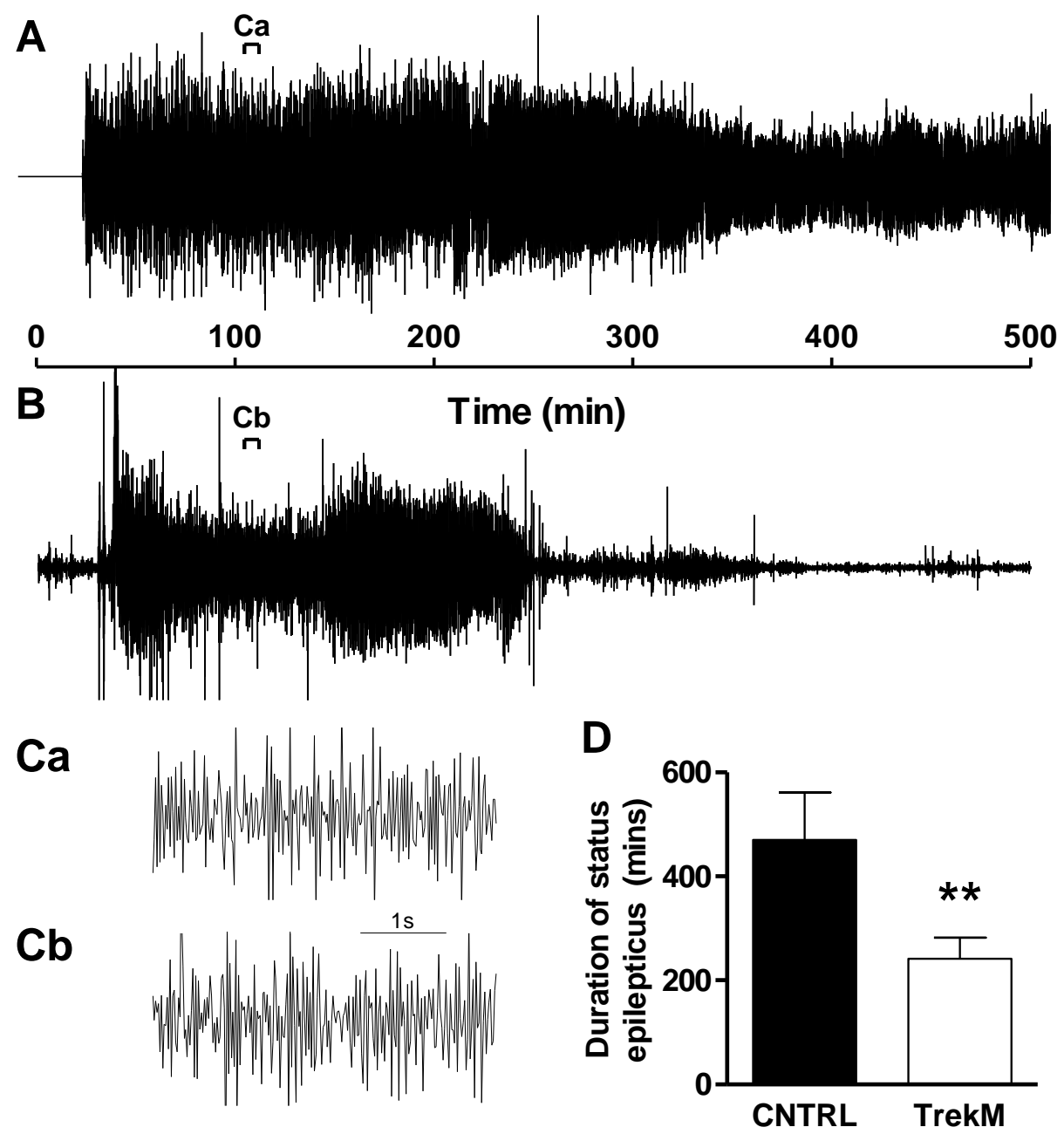

Figure 7A: Effect of pre-injection of AAV-Ctrl and AAV-TREK-M virus in pilocarpine induced status epilepticus in rats.

A: EEG trace of status epilepticus in an AAV-Ctrl pre-injected rat.

B: EEG trace of status epilepticus in an AAV-TREK-M pre-injected rat. Both A and B traces are of 13 hours EEG recording.

C: EEG traces of rat injected with control virus (Ca) and rat injected with TREK-M virus $(\mathrm{Cb})$.

D: Status epilepticus duration is significantly decreased in rats pre-injected with AAVTREK-M compared to rats pre injected with control virus. Analysis is done by using one tailed non-parametric t-test (Mann-Whitney test). ${ }^{* *} \mathrm{p}<.005$ 


\subsection{TREK-M protected against neuronal death}

We hypothesized that the ability of TREK-M to reduce firing would also render neuronal protection from pilocarpine induced excitotoxicity. We measured neuronal death by using Fluoro-Jade-C. Fluoro-Jade C is a Nissl stain which has proven to be a reliable marker for neuronal degeneration (Blakemore, Wang et al. 2008). TREK-M (scAAV5-GABRA4TREK-M) pre-injected rats $(n=4)$ and control rats $(n=4)$ were perfused after 48 hours of SE induction. Horizontal slices were cut and staining was performed on them. The slices are chosen so that they are all collected from the same depth close to injection track. The slices were counterstained with DAPI. Representative images of Fluoro-Jade C stained brain slices from different regions like EC, CA3, CA1 and DG are represented in figure 8. We found that scAAV5-GABRA4-TREK-M pre injected rats had significantly less Fluoro-Jade $\mathrm{C}$ positive cells per brain slice in the entorhinal cortex (Fig 8A), CA3 (fig 8C) and CA1 (fig 8E) regions compared to control viral particles pre-injected rats EC (fig 8B), CA3 (fig 8D) and CA1 (fig 8F). However, no reduction was observed in FJC+ cells in the dentate gyrus, where neuronal death predominantly occurs in the hilus as others have observed previously (Wang, Ghosh et al. 2008;Sun, Mtchedlishvili et al. 2007). In order to quantify the number of dead cells which is represented by green colored FluoroJade $-\mathrm{C}$ stained neurons, we calculated the Fluoro-Jade $\mathrm{C}$ positive neurons in each brain slice. Significantly less Fluoro-Jade C positive neurons per slice were seen in EC (fig 9A) CA3 (fig 9B), CA1 (fig 9C). No difference in neuronal death was seen in DG (fig 9D). These results indicate that TREK-M was partially able to protect neurons from death in regions where the AAV was injected and that this effect was extended to the CA1 region. 


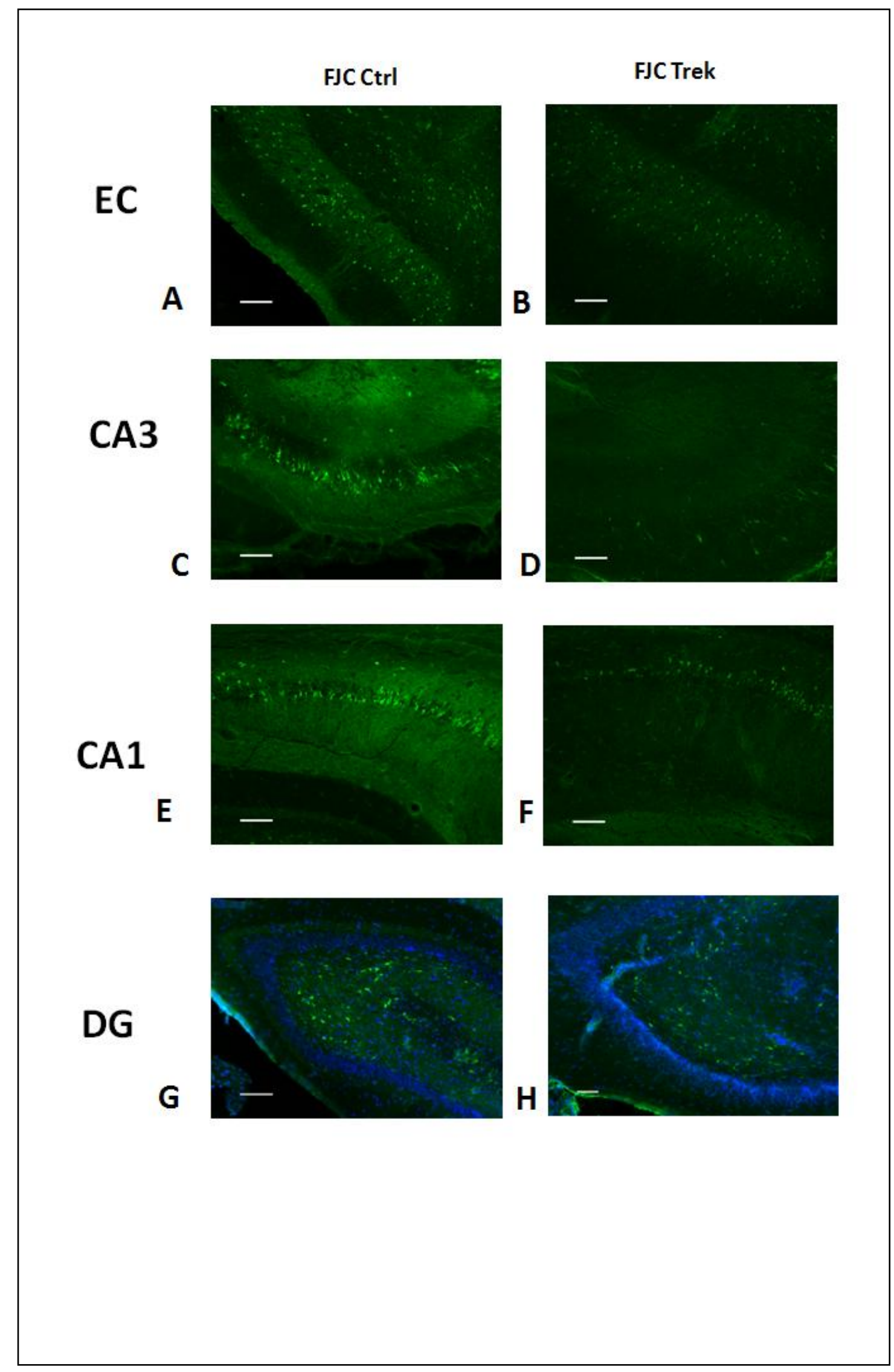


Figure 8: Effect of pre injection of AAV-CTRL and AAV-Trek on neuronal death in pilocarpine induced epileptic rats.

Typical examples of Fluorojade $C$ labeling of degenerated neurons in EC (A), CA3(C), CA1(E) and DG $(\mathrm{G})$ layers respectively of AAV-CTRL pre injected epileptic rats.

Typical examples of Fluorojade $\mathrm{C}$ labeling of degenerated neurons in EC (B), CA3(D), CA1(F) and DG (H) layers respectively of AAV-TREK-M pre injected epileptic rats 


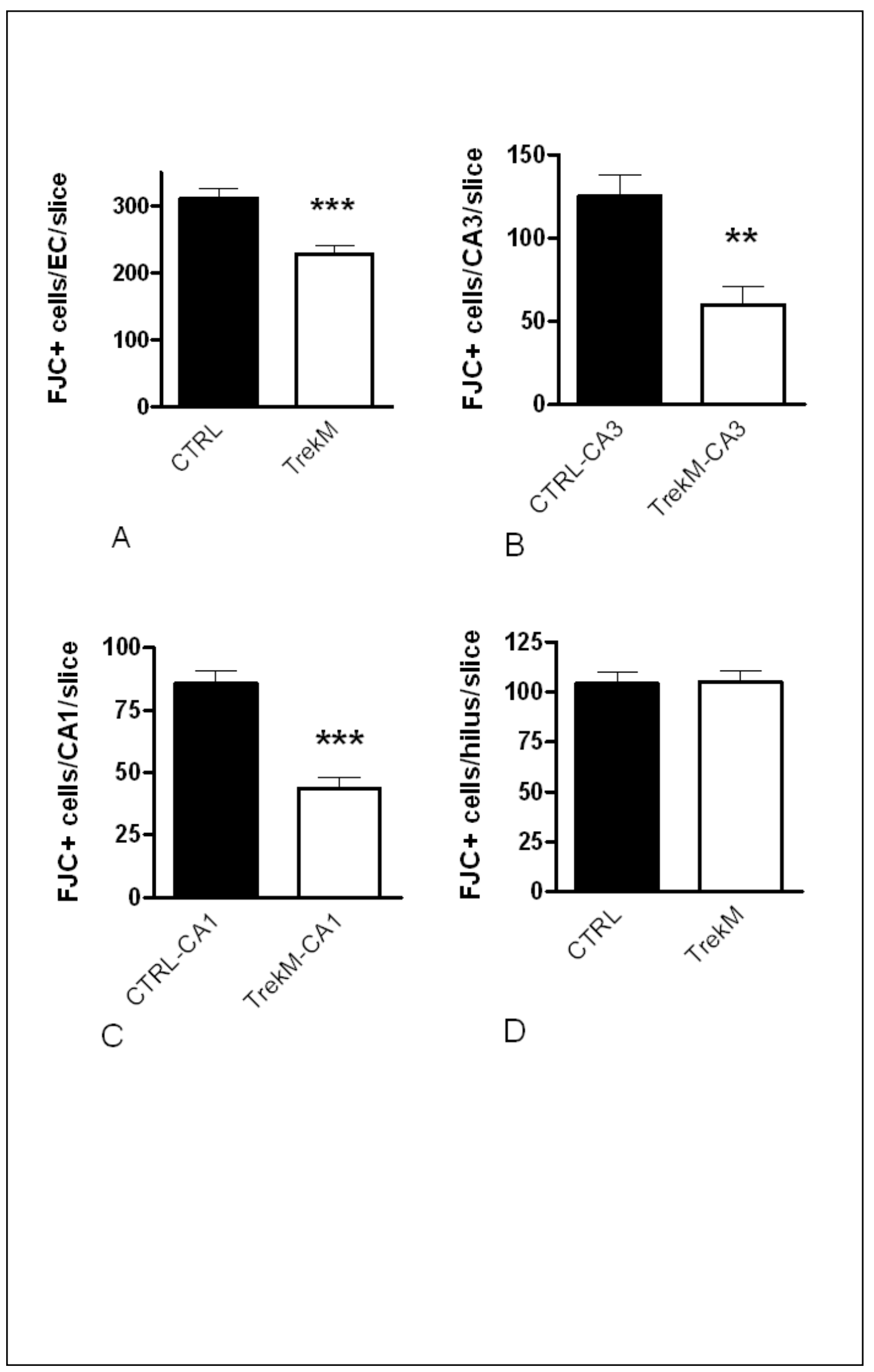


Figure 9: Changes in the number of dead neurons in the EC, CA3, CA1 and DG regions of AAV-TREK-M pre injected epileptic rats compared to that of AAVCtrl pre injected rats.

A: There is significant less neuronal cell death in the EC region of rats preinjected with AAV-TREK-M versus AAV-CTRL pre injected epileptic rats; *** $\mathrm{p}<.05$ vs control .

$\mathrm{B}$ : There is significant less neuronal death in the CA3 region of rats preinjected with AAV-TREK-M versus AAV-CTRL pre injected epileptic rats; ** $\mathrm{p}<.05$ vs control.

$\mathrm{C}$ : There is significant less neuronal death in CA1 region of rats preinjected with AAV-TREK-M versus AAV-CTRL pre injected epileptic rats; *** $\mathrm{p}<.05$ vs control .

D: No significant less neuronal death is observed in DG region of rats preinjected with AAV-TREK-M versus AAV-CTRL pre injected epileptic rats. 


\section{DISCUSSION}

This study is focused on finding a possible alternative gene therapy treatment for SE by using mutated potassium leak channel TREK-M as the gene of choice. This study provides the rationale for using TREK-M to treat TLE. Recombinant adeno-associated virus (rAAV) was chosen as the vector of choice because it is safe and non-toxic in human tissue. The neuronal promoter GABRA4 was used to direct the expression of TREK-M gene, which was packaged in a scAAV construct. The effect of TREK-M in status epilepticus was assessed in a pilocarpine-induced rat model of epilepsy. Three main conclusions can be drawn from these experiments. First, enhanced GABRA4 promoter retained its neuronal specificity even after modifying it with a CMV enhancer at the 5' end and a CMV minimum sequence at its 3' end. Second, TREK-M ameliorates status epilepticus in pilocarpine induced epileptic rats. Third, significantly less neuronal death was observed in CA3, CA1 and entorhinal cortex (EC) regions of the brain of TREK-M pre-injected rats compared to controls.

When choosing neuronal promoter for directing the expression of TREK-M in epileptic rats, the GABRA4 promoter has emerged as top choice because of the multiple factors. First, GABA is one of the main inhibitory neurotransmitters in the brain. This effect is mediated by activation of $\mathrm{GABA}_{\mathrm{A}}$ receptors. $\mathrm{GABA}_{\mathrm{A}}$ receptors are composed of six $\alpha$ subunits, four $\beta$ subunits, three types of $\gamma$ subunits and one of each $\delta, \theta, \varepsilon$ and $\pi$ subunits (Macdonald and Botzolakis 2009).GABRA4 is the human promoter for the $\alpha 4$ subunit (Roberts, Raol et al. 2005). The dentate granule cells in the hippocampus show high expression of $\alpha 4 \mathrm{GABA}_{\mathrm{A}}$ subunit receptors (Sun, Sieghart et al. 2004). Studies have shown that $\alpha 4$ subunit expression increases in the dentate gyrus of epileptic rats (Raol, 
Zhang et al. 2006). Also, increased expression of GABRA4 has been observed in rats which have undergone electroshock (Ma, Song et al. 2004). The human and rat GABRA4 promoters have consensus binding sites for early growth response (Egr) transcription factor 3 (Egr3). The expression of Egr3 is also upregulated in the dentate gyrus after seizures (Roberts, Raol et al. 2005). Second, the GABRA4 promoter is 542 bp long which is useful to fit in the $2.4 \mathrm{~kb}$ packaging size of self-complementary scAAV virus. We modified the GABRA4 promoter to enhance its power since the original promoter had a very weak activity. The power of the promoter was enhanced by 50 -fold by adding a CMV enhancer sequence at its 5' end and a CMV minimum sequence at its 3' end. Our results have shown that the GABRA4 promoter enhanced by both of these elements showed highest expression of Venus fluorescent protein in ND7 cells without losing its neuronal specificity. In order to test that the modified GABRA4 promoter did not lose its neuronal specificity non neuronal cells (HEK-293 cells) and neuronal cells (ND7 cells) were transfected with the enhanced GABRA4 promoter directing the expression of Venus fluorescent protein. The expression of the Venus protein was only found in ND7 cells and not in Hek-293 cells, confirming that the modified GABRA4 promoter still retained its neuronal specificity.

Entorhinal cortex (EC) neurons via the perforant path and temporoammonic path form the major excitatory input to the dentate gyrus (DG), CA3 and CA2 regions of the brain (Jones and McHugh ; Segal and Landis 1974; Steward and Scoville 1976). Studies have revealed that hyperexcitability in EC lead to hippocampal hyperexcitability which further excites hippocampal-EC circuit due to reciprocal connections (Buckmaster and Dudek 1997; Kobayashi, Wen et al. 2003). The layer II neurons of the EC become hyper- 
excitable in animal epilepsy models. Stimulation of the deep layers of the EC shows long excitatory synaptic responses in layer II neurons (Bear, Fountain et al. 1996; Scharfman 1996; Tolner, Kloosterman et al. 2005; Kumar and Buckmaster 2006). Entorhinal cortex volume is significantly decreased in patients suffering from TLE (Jutila, Ylinen et al. 2001; Bartolomei, Khalil et al. 2005. Studies have shown that stimulating only the EC can evoke spontaneous interictal spikes in the hippocampus \{Rutecki, 1989 \#10999). Seizure suppression has been successful in TLE patients by surgical removal of hippocampus or EC (Siegel, Wieser et al. 1990). Therefore, we chose EC as one of our site of injections in order to get maximum infection in the trisynaptic circuit. CA3 pyramidal cells have a tendency to generate seizures (McCormick and Contreras 2001). The neurons in this region can have the ability to generate intrinsic bursts of 2-5 action potentials at 200-350 Hertz (McCormick and Contreras 2001). The dentate granule cells of the dentate gyrus project mossy fibers connecting with the pyramidal cells of the CA3 field of the hippocampus. The pyramidal cells of the CA3 project to the CA1 layer through the Schaffer's collateral axons. A previous finding suggests that dentate granule cells act as a gate keeper for spread of seizures. The mossy fibers from these cells sprout in TLE patients and extend to other hippocampal regions forming new synaptic connections (Buckmaster, Zhang et al. 2002). For this reason, we also chose CA3 as a second injection site. Before delving into in vivo studies, we wanted to validate the site of injection of viral particles into the brain of rats. To do so, naïve rats were injected with AAV5-CMV-GFP viral particles in the CA3 and EC brain regions. Fluorescent images of brain slices revealed robust GFP expression in the dentate granule neurons, and layers II, 
IV and V neurons in the EC. These findings validated the site of injections to target the chosen brain regions of rat.

Our results demonstrate that naïve rats when pre injected with the scAAV-GABRA4TREK-M viral particles spent significantly less time in status epilepticus compared to rats injected with control viral particles. This suggests that TREK-M expression in EC neurons and dentate granule neurons reduce epileptic discharges. The total time of acute seizures of scAAV-GABRA4-TREK-M pre injected rats was also significantly lower than in control rats. However, we did not find any difference in the behavior stage scores of experiments versus control rats.

Pilocarpine-induced status epilepticus causes neuronal death in the rodent brain (Benkovic, O'Callaghan et al. 2004; Wang, Liu et al. 2008). Pilocarpine is a muscarinic agonist which activates M1 muscarinic receptors directly. Studies on M1 receptor knockout mice have shown that pilocarpine is unable to produce seizures in these animals (Hamilton, Loose et al. 1997; Curia, Longo et al. 2008). This suggests that status epilepticus is generated as a result of an imbalance created by pilocarpine between excitatory and inhibitory transmission. Also, an increase in glutamate levels in the hippocampus has been reported following in vivo micro dialysis of pilocarpine (Curia, Longo et al. 2008). This increased glutamate level results in excitotoxic neuronal death (Greene and Greenamyre 1996). In order to study neuronal death in brain tissue we performed Fluoro-Jade C staining. Previous studies have shown Fluoro-Jade labeled neurons in $\mathrm{CA} 3, \mathrm{CA} 1$ and dentate gyrus in pilocarpine injected epileptic rats (Poirier, Capek et al. 2000). Our results show that rats when pre-injected with AAV-GABRA4TREK-M viral particles have significantly less death in the CA3, CA1 and EC regions of 
the brain compared to controls. This result suggests that TREK-M expression in neurons adjacent to the site of injection is less prone to neuronal death by pilocarpine-induced status epilecticus (see Appendix Fig 2). The mutated potassium channel incorporation into the neurons prevents the plasma membrane to shoot repetitive firing and as a result neuron death is prevented too. The reason to choose Fluoro-Jade $\mathrm{C}$ is because it has much stronger affinity for degenerating neurons than Fluoro-Jade B and it stains with "highest resolution" (Schmued, Stowers et al. 2005). Fluoro-Jade C stains all dead neurons no matter whether it had died by apoptosis or necrosis (Wang, Liu et al. 2008). The exact mechanism by which this stain binds with degenerating neurons is still not clear. One possible way might be that strong acidic Fluoro-Jade $\mathrm{C}$ reacts with a strong basic molecule that is presented by a degenerating neuron (Schmued, Albertson et al. 1997). Degenerating neurons caused by kainic acid insult express a cleaved microtubular protein called c-tau (Zemlan, Mulchahey et al. 2003) which might bind to Fluoro-Jade C. It is a very specific stain for degenerating neurons (Schmued, Stowers et al. 2005). However, some astrocyte labeling by this stain has been reported. GFAP expression confirmed that the labeled astrocytes are activated cells rather than dead astrocytes. In some control tissue labeling of red blood cells, choroid plexus and collagen fibrils of meninges also showed positive Fluoro-Jade C staining. Fluoro-Jade C is extremely photostable and lasts for at least a year (Schmued, Stowers et al. 2005).

Temporal lobe epilepsy is a good candidate for gene therapy as it is widely studied in animal models of epilepsy. Also, various candidate genes for therapeutic targeting have been identified for this disorder from surgically resected tissue (Vezzani 2004; Riban, Fitzsimons et al. 2009). Gene therapy is beneficial because it allows specific targeting of 
a region of interest without causing much harm to healthy surrounding tissue and fewer side effects compared to surgical resection of hippocampus and anti-epileptic drug administration. Gene therapy for temporal lobe epilepsy will be more effective compared to the only treatment option left for TLE patients, surgery. Currently, the most effective treatment is surgical removal of the hippocampus and other associated limbic structures like the amygdala and entorhinal cortex. However, surgical treatment fails in $20-30 \%$ of the patients (Renate M. Kalnins1 2004; Adil Harroud 2012). In most patients, seizures arise in the mesial temporal lobe. In these patients sometimes it is not possible to surgically resect the mesial temporal lobe structures because of 'imprecise delimitation' or 'surgical limitation'. In $10 \%$ of TLE patients, seizure focus has been found in the lateral temporal lobe (Schramm, Kral et al. 2001). In some patients within this category, the 'epileptogenic zone' consisted of superior temporal gyrus which required anterior temporal lobectomy. Even this procedure does not guarantee full cure for these patients. In some patients seizures relapsed in lateral neocortex. 'Dual pathology' involving both mesial temporal lobe sclerosis and extrahippocampal lesion is another reason for surgical failure. Even after surgical resection of one hippocampus, relapse occurs in the contralateral side or extra temporal zone (Adil Harroud 2012). Furthermore, surgery is not hundred percent effective and comes with serious side effects like loss of new memory formation, verbal and visual memory impairment, etc. Studies have shown that patients who undergo left temporal lobe resection suffer from verbal memory loss, while right temporal lobe resection leads to visual memory loss. TLE patients with bilateral temporal lobectomy suffer high memory loss and significant effect on intellect and language (Bell, Lin et al.). Surgical side effects also depend on degree of sclerosis in 
different patients and seizure onset age. TREK-M delivery into hyper-excitable neurons via scAAV5 viral vector has clearly shown an effect in decreasing significantly status epilepticus time in a model of pilocarpine-induced epileptic rats. Also these rats have significantly less neuronal death compared to controls. AAV mediated gene therapy clearly can emerge as one of the alternative treatments for temporal lobe epilepsy and TREK-M can be used as a gene of choice for treating the neurological disorder.

Enhancing $\mathrm{K}+$ currents to treat epilepsy is a mechanism of action of retigabine (ezogabine; Potiga) (Gunthorpe, Large et al. 2012). The hypothesis that overexpression of $\mathrm{K}+$ channels can be used as a therapeutic treatment on focal epilepsy has been supported in animal models of tetanus toxin-induced epilepsy (Wykes, Heeroma et al. 2012). In conclusion, our results demonstrate that AAV-TREK-M might provide a novel gene therapy treatment for refractory status epilepticus, and further pre-clinical studies are needed to study its effect on spontaneous seizures in chronic models of TLE. 


\title{
CHAPTER 3: Testing drug-regulated expression of TREK-M on spontaneous seizures in the rat lithium-pilocarpine model of temporal lobe epilepsy
}

\begin{abstract}
Temporal lobe epilepsy (TLE) affects millions of people worldwide and common form of adult, focal, refractory epilepsy. The focus of this study was to investigate the effect of drug regulated expression of TREK-M on spontaneous seizures in the lithium pilocarpine model of TLE. The addition of drug regulation to gene therapy will endow doctors to titrate the beneficial effect and minimize possible side effects. In our lab, we have developed the first AAV targeting vector that allows doxycycline to turn on the expression of two genes of interest. We have used one promoter to drive the expression of a reverse tetracycline repressor protein that is fused to the strong transcriptional activator VP16 (rtTR/VP16). Binding of doxycycline to rtTR/VP16 activates the transactivator complex. This transactivator complex in turn binds to 7 copies of tetracycline operator sequence (tetO) to activate gene expression. Doxycycline is approved by the FDA for human use and enters the brain (Stieger, Belbellaa et al. 2009). Our results demonstrate robust doxycycline regulation of TREK-M expression, with little background expression in the absence of doxycycline. Doxycycline regulated expression of TREK-M caused a significant decrease in spontaneous seizures in chronic TLE rats. This study shows promising results for developing an alternative adeno-associated virus mediated gene therapy treatment for pharmacoresistant focal epilepsies for patients who either do not want or cannot be treated by surgical resection.
\end{abstract}




\section{MATERIALS AND METHODS}

\subsection{Adeno-associated virus constructs}

The TREK-M cDNA was subcloned into a custom designed AAV targeting vector. The AAV targeting vector contained the 500 bp human synapsin I promoter (plus the CMV minimum 3' leader sequence driving the expression of the doxycycline-on transcriptional activator rtTA-VP16. It also contained the $7 \mathrm{x}$ tet operator sequence driving mCherry. This base vector was extensively tested in cultured neuroblastoma and hippocampal neurons for appropriate doxycycline expression (data not shown). Using appropriate restriction enzymes a homemade internal ribosome entry signal (IRES; derived from elongation factor $1 \alpha$; Arias and Perez-Reyes, unpublished observations) and TREK-M cDNAs were added. Addition of eIRES between mCherry and TREK-M results in transcription of both proteins from a single mRNA, therefore, expression of mCherry provides a faithful readout of TREK-M protein expression. This assumption was verified by transfecting HEK-293 cells, then recording TREK-M currents from red fluorescent cells (Appendix 4). The AAV targeting vector was assembled by ligation of these inserts, verified by restriction mapping, and then grown in a large scale DNA preparation. To rule out possible rearrangements, the large scale DNA preparation was sequenced (Genewiz). It was then sent to the University of North Carolina Vector Core for packaging into AAV serotype 5 particles. The plasmid map of AAV5-SmonCeiT is shown in figure 11. 


\subsection{Chronic rat model of TLE}

. Rats were injected with lithium (3mmol/kg; Sigma). This pre-injection of lithium potentiates the seizure inducing action of pilocarpine (Jope, Morrisett et al. 1986). Twenty hours after lithium injection, scopolamine $(1 \mathrm{mg} / \mathrm{kg})$ was injected into the rats which reduces peripheral effects due to pilocarpine and reduces mortality (Lemos and Cavalheiro 1995). Forty minutes later, pilocarpine (50mg/kg; Sigma) was injected. The

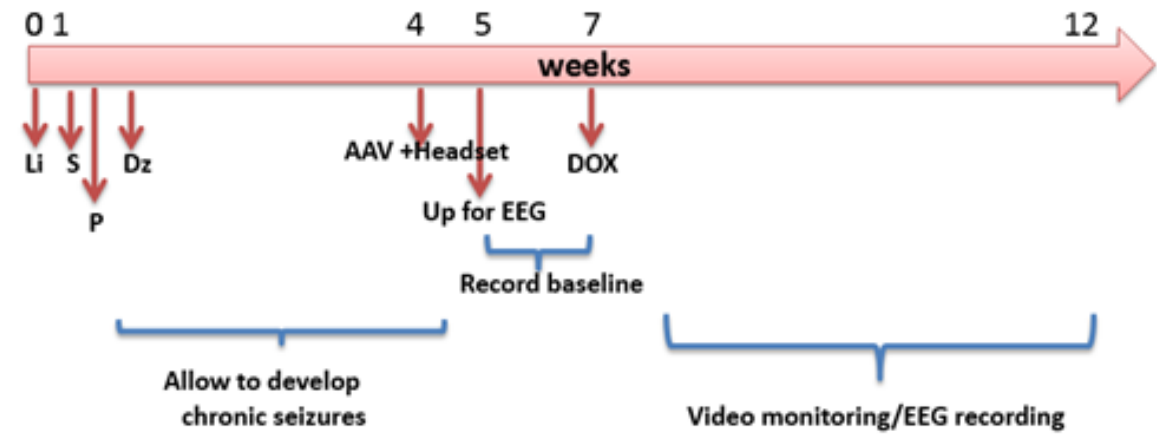

Li (Lithium) - sensitizes rat to pilocarpine

$S$ (Scopolamine $)=$ reduces peripheral side effects

$P($ Pilocarpine $)=$ induces seizures

Dz $($ Diazepam $)=$ terminated behavioral seizures, reduces mortality

Figure 10: Time-line of lithium pilocarpine chronic epilepsy model of rat.

The plan for the chronic epileptic rats is shown in this figure. The rats were made epileptic by intraperitoneal injection of pilocarpine. All other injections shown in the figure were done intraperitoneally. After they developed chronic seizures, AAV virus was injected into the CA3 and EC region of the brain and headset were implanted.

The rats were allowed to recover for 1-2 weeks and then the baseline data were collected. Subsequently, doxycycline was given in the drinking water. 
rats were injected with diazepam (10mg/kg; Sigma) after 2 hours of pilocarpine injection in order to stop the convulsions and reduce mortality. All drugs were injected intraperitoneally. These rats were allowed to develop chronic seizures for 4 weeks. Seizures were detected visually. The time-line of this chronic model of epilepsy is shown in figure 10.

\subsection{Stereotaxic brain injections}

AAV injections were made into chronic epileptic rats that had been observed to have spontaneous seizures. Rats were anesthetized with isoflurane (Sigma), given eye lubricant, and placed on a heated pad inside a stereotactic frame (David Kopf Instruments Model 940 with digital display). The rats were subjected to continuous isoflurane (EZB800, World Precision Instruments, Inc) for the entire surgery. They were continuously monitored for anesthesia depth for the complete surgery at every 10 minutes. An incision was made on the head and all the muscles and underlying tissues were cleared to expose the bony skull for brain injections and headset mounting. We used a mounted stereotaxic drill (Model 1474, David Kopf Instruments). The injection co-ordinates were the same used in the acute studies of Chapter 2 ( $\mathrm{mm}$ from bregma): entorhinal cortex, lateral 5, posterior -8.3 ; and depth from -6.6 to 5.6; CA3, lateral 4.6, posterior -4.4, and depth from -6.6 to -5.1 (Paxinos, Watson et al. 1980). Seven additional burr holes were drilled into the skull. Four of these were used to put bone anchor screws in order to fix the electroencephalogram (EEG) headset on the head, whereas the other three burr holes were used to place two cortical electrodes and one depth electrode. The depth electrode was made from perfluoroalkoxy-insulated silver wire ( 0.008 " diameter, A-M systems) and placed in the piriform cortex at the following coordinates ( $\mathrm{mm}$ from bregma): lateral 5, posterior 
-0.3 , and depth -9.5 . We injected $3 \mu 1$ of virus (serotype 5 ; titer $10^{\wedge} 12$ particles $/ \mathrm{ml}$ ) at a rate of $20 \mathrm{nl} / \mathrm{sec}$ into each site with a $10 \mu \mathrm{l}$ Hamilton syringe connected to an infusion pump controlled by a microprocessor. In order to allow diffusion from the site of injection, the injector was left in place for two minutes after virus infusion. After virus injection, the electrodes were placed in the designated burr holes and the headset was fixed to the head by applying dental cranioplast. The skin was sutured once the cranioplast dried and set. The rat was given an intraperitoneal injection of $0.1 \mathrm{ml}$ ketoprofen for analgesia. Local application of lidocaine was also used around the suture. The rats were allowed to recover on a heated pad after surgery and then returned to the vivarium once they were fully awake. The rats were allowed to recover from surgery for a week after which they were connected to the EEG recording system. All procedures

were approved by the Institutional Biosafety Committee (IBC) and the Institutional Animal Care and Use Committee (IACUC).

\subsection{EEG monitoring}

The rats were video monitored and their EEG was recorded for 24 hours. 7 days a week. Individual seizures were marked on the recording and their corresponding behavioral stage (BSS) were scored by inspecting the video recording. The BSS scoring scale was explained in chapter 2 of this thesis (methods chapter 2). EEGs were recorded using the Grass Neurodata Acquisition System (Grass Instruments Co., Quincy, Mass, USA) and were analysed using Stellate Reviewer software. 


\subsection{Data analysis}

The seizure frequency of each rat was measured. Data were exported from Stellate Reviewer software and into Excel. The average duration and summed seizure frequency per day was calculated in Excel.

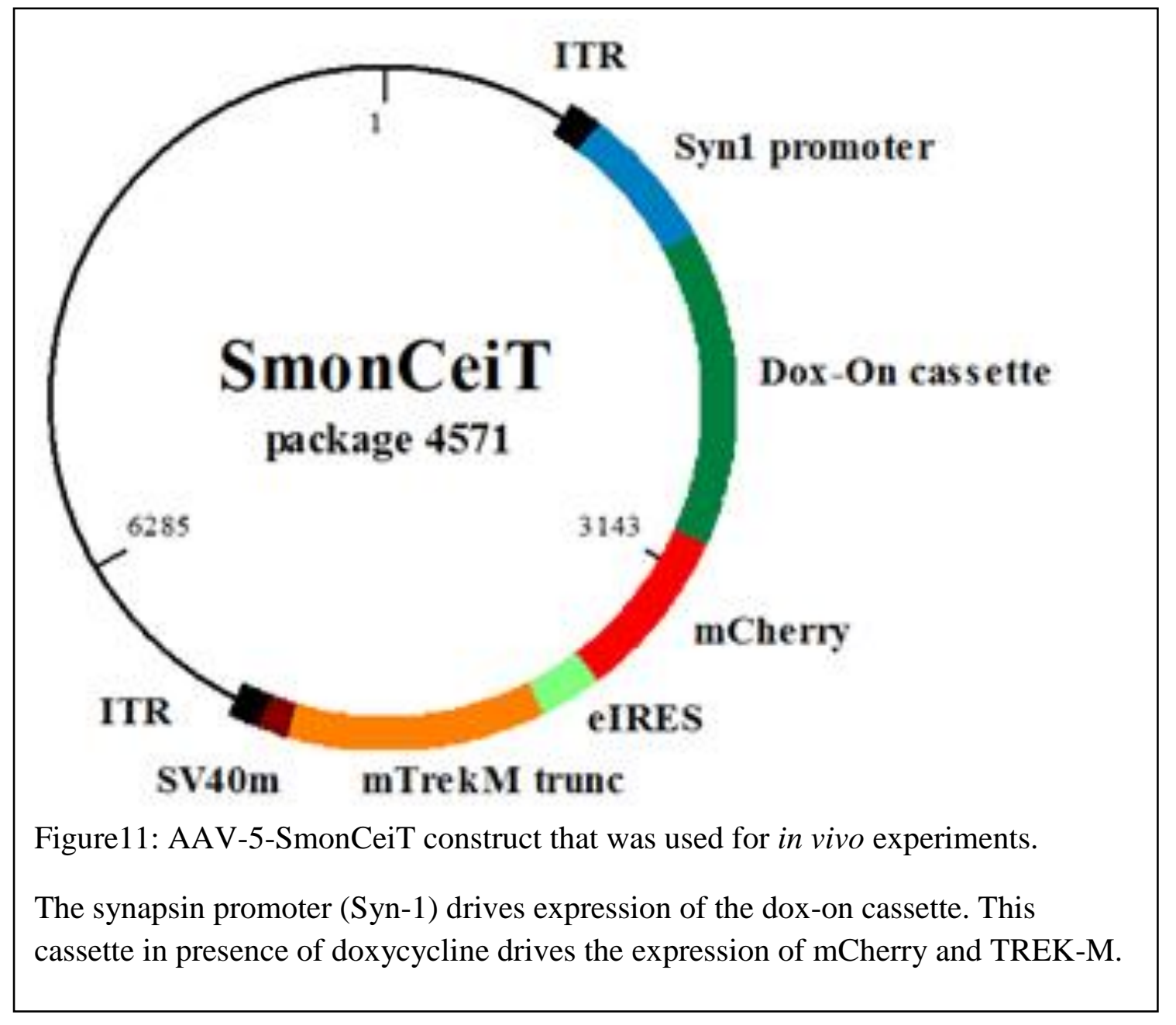




\section{RESULTS}

\subsection{Doxycycline robustly regulates TREK-M expression}

Two chronic female epileptic rats were injected with doxycycline-regulated TREK-M virus bilaterally into the $\mathrm{EC}$ and $\mathrm{CA} 3$ region of their brain. One rat was provided with doxycycline $(200 \mu \mathrm{g} / \mathrm{ml})$ in the water for two weeks. Both rats were perfused with paraformaldehyde, the brain excised, sliced, then analyzed by fluorescent microscopy. Robust mCherry expression was observed in the EC and CA3 of the rat which had doxycycline in its drinking water (Fig. 9A). Very low expression of mCherry was found in the EC and $\mathrm{CA} 3$ of the rat that did not receive doxycycline in its drinking water (Fig. 12A). Representative images and quantification of mCherry fluorescence has been represented in figure 12B. The methods used to quantify mCherry expression were similar to those used for Fluoro-Jade C labeling in Chapter 2. Briefly, we used an autosegmentation algorithm that uses the midpoint of the background signal, contour

mapping, and an area limit of 2. Expression of mCherry was 39-fold higher in the EC of the rat drinking doxycycline water compared to the rat drinking normal water (Fig.12C). 


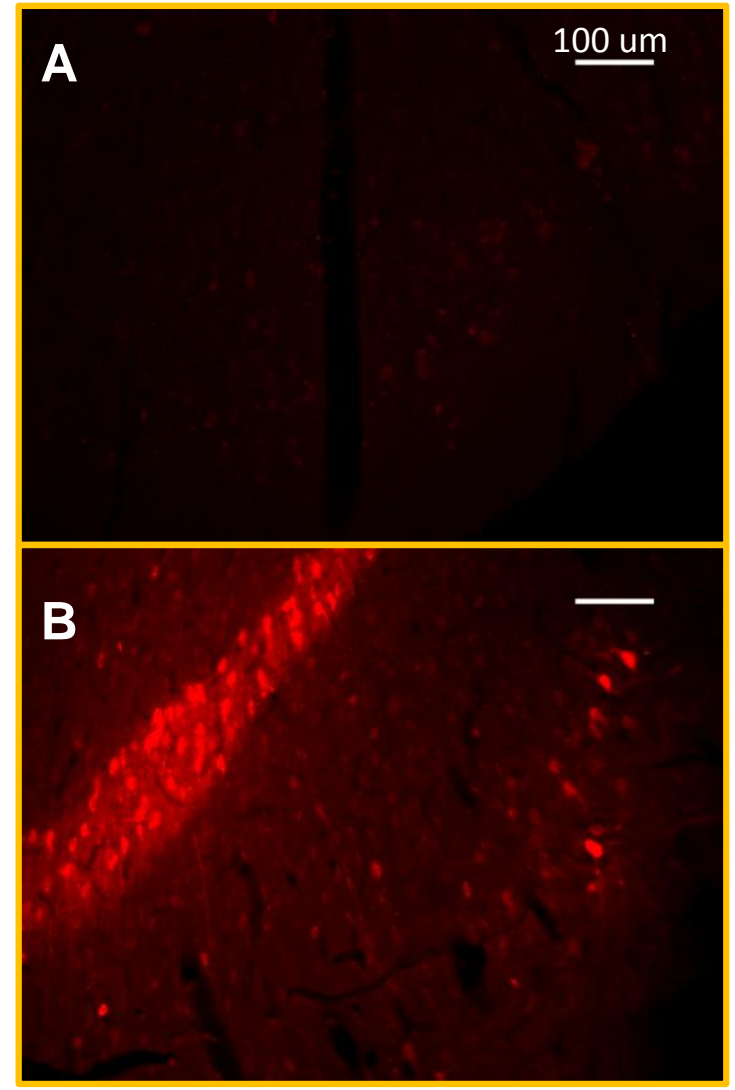

C

Sm on CeiT A A V 5

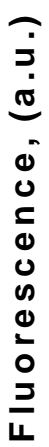

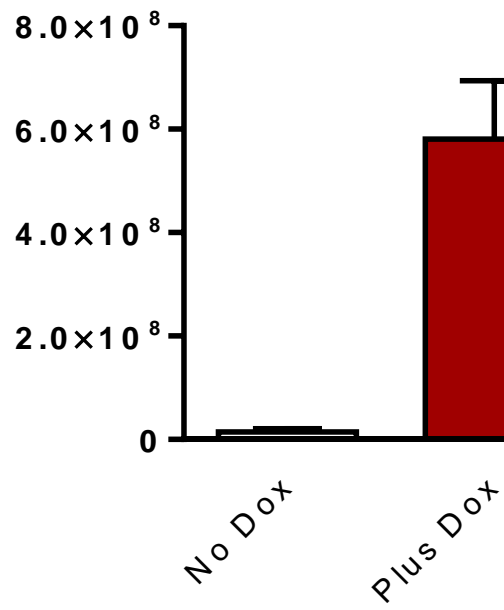

Figure 12: Validation of doxycycline regulated expression of AAV5-SmonCeiT virus after intraparenchymal injection into the $\mathrm{CA} 3$ and entorhinal cortex (EC) of chronic epileptic rats. AAV5-SmonCeiT virus was injected bilaterally into CA3 and EC region of two female chronic epileptic rats. One rat received doxycycline water for two weeks. They were then perfused with $4 \%$ paraformaldehyde. The brains were sliced horizontally and pictures were shot using IPLAB software, using Semrock red fluorescent cubes State excitation and emission wavelengths.

A: 10x image of the EC region of the rat brain who did not receive doxycycline water. Very little background mCherry expression was seen in the EC.

B: 10x image of EC region of the rat brain who received doxycycline water. Robust mCherry expression was seen in the layer IV of EC.

C: Analysis of mCherry expression was done by IPLAB software by autosegmentation. Doxycycline-regulated mCherry expression was 39 fold higher in the EC of rat drinking doxycycline water than the rat drinking normal water. 


\subsection{Doxycycline regulated expression of TREK-M effects seizure frequency of chronic epileptic rats}

The experiment was started by injecting ten rats with pilocarpine and monitoring their behavioral seizures. All rats developed BSS level 5 bilateral tonic clonic seizures that lasted approximately 90 minutes. As in the acute studies, motor seizures were terminated with diazepam injection 2 hours after pilocarpine administration. Within the first couple of weeks after pilocarpine injection four rats died. Six rats went on to develop spontaneous seizures. One month after pilocarpine injection they were injected with doxycycline-regulated TREK-M viral particles (AAV5-SmonCeiT) bilaterally either into the $\mathrm{EC}$ or both in $\mathrm{EC}$ and $\mathrm{CA} 3$. After recovering from surgery they were tethered to the EEG system and continuously video/EEG monitored. One of the rats did not seize at all when we started data collection and another one lost its headset after first doxycycline dose. The seizure diaries of the rats DP25, DP24, DP23 and DP22 have been shown in Fig $13 \mathrm{~A}, \mathrm{~B}, \mathrm{C}$ and $\mathrm{D}$ respectively. The shaded region corresponds to the time when doxycycline $(200 \mu \mathrm{g} / \mathrm{ml})$ was added in their drinking water. We planned to give doxycycline to each rat and then measure wash-out to verify that any change in seizure frequency correlated with doxycycline-regulated expression of TREK-M. This cross-over design minimizes the unlikely occurrence of spontaneous remission. We planned to perform three doxycycline-on periods separated by wash-out. Most animals lost their headset sometime during the experiment, with only DP25 making it desired point of the experiment. Rats DP23, DP24, and DP25 showed an initial spike in seizure frequency upon first administration of doxycycline. We followed a protocol from the literature where doxycycline, a slightly bitter substance, is diluted in $5 \%$ sucrose. Therefore, it is 
not possible to ascribe the jump in frequency to doxycycline. Therefore, we cut short the first doxycycline dosing period and acclimatized the animals to sucrose water. The data from this truncated treatment period was excluded from subsequent analysis. Subsequently we treated the rats with doxycycline in the same concentration of sucrose. DP24 and DP25 showed a clear drop in seizure frequency to this second treatment. DP22 showed no response. Notably, this rat developed a high seizure frequency before any treatment, indicating a profound increase in the excitability of epileptic circuits. The best responder was DP25, and a bar graph of his seizure frequency during the treatments is shown in figure 13E. The seizure frequency was significantly decreased by doxycycline $\left({ }^{* *} \mathrm{p}<.005\right)$. We also analyzed the inter-seizure intervals, finding a significant increase in DP25, DP24, and DP23 (data not shown). Together, these results indicate that TREK-M expression regulated by doxycycline was successful in treating spontaneous seizures in the pilocarpine model of TLE. 

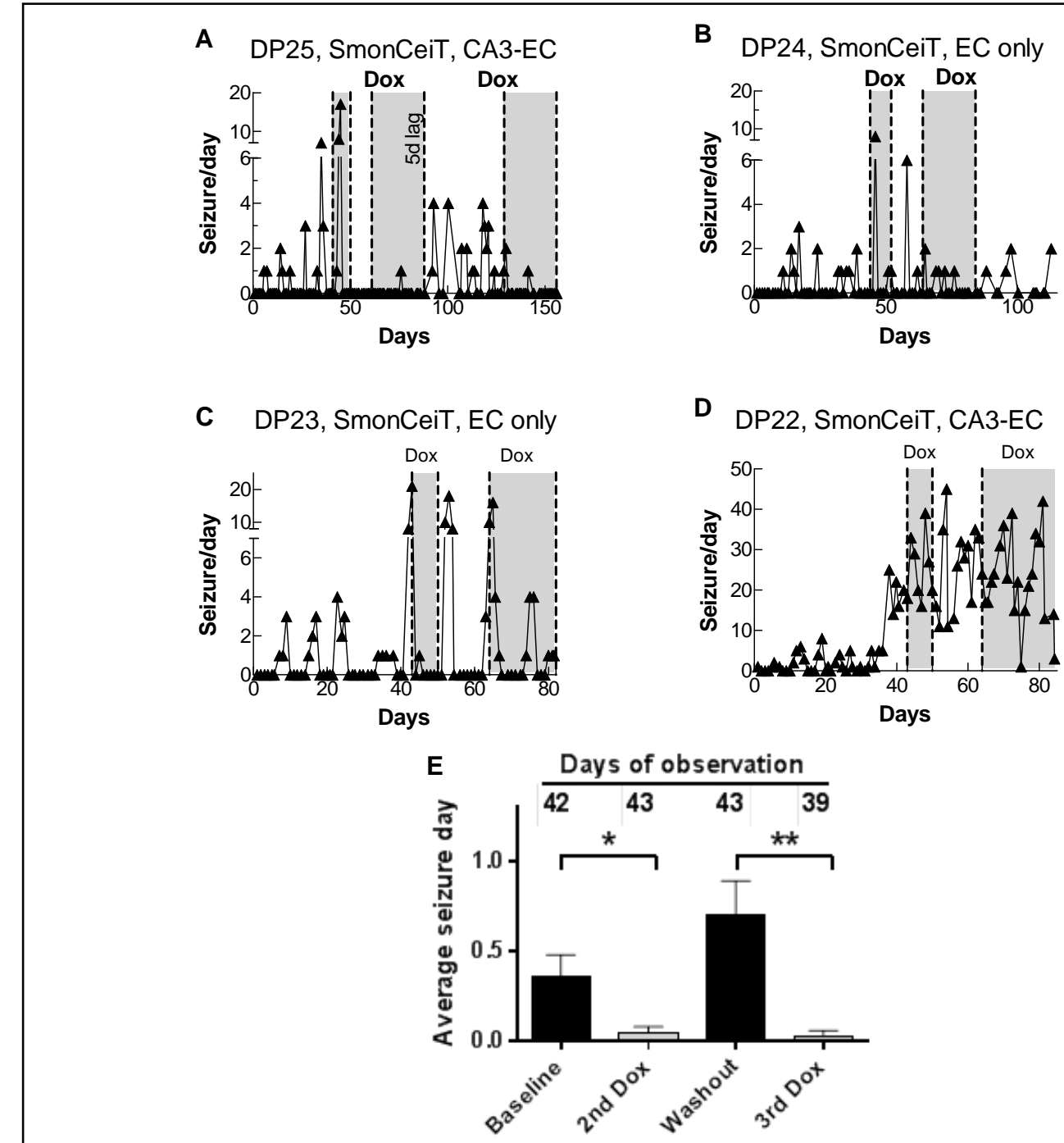

Figure 13: Seizure diaries corresponding to rat DP25 (A), DP24 (B), DP23 (C) and DP22 (D). They were injected with AAV5-SmonCeiT virus bilaterally either into EC or in EC and CA3. The shaded regions indicate the time period when doxycycline was given in its water. Day zero is the day video and EEG monitoring was started.

E: Bar graph of seizure frequency of DP25. The seizure frequency was significantly decreased between baseline and second dox dose; $* \mathrm{p}<.05$. The seizure frequency between wash-out and third dox dose was also significantly reduced; **p<.005. No significant difference was seen between baseline and wash out seizure frequency. 


\subsection{Doxycycline regulated TREK-M expression significantly decreased the normalized seizure frequency of chronic epileptic rats}

Since each animal varied in seizure frequency, we developed a method to normalize the data. The dominator for the normalization was the average of the seizures/day for each rat during the baseline and washout periods. The numerator was the average of the seizures/day for each rat during the second, and where possible the third doxycycline treatment period. The normalized seizure frequency of DP25, DP24, DP23 and DP22 has been plotted in Figure 14A. The normalized seizure frequency of these rats significantly decreased when they were on doxycycline water compared to normal water (Fig.12B). Out of these 4 rats, normalized seizure frequency of DP25 was lowest suggesting it responded very well to the Dox treatment. The normalized seizure frequency DP24 and DP23 decreased 50\% during Dox treatment, suggesting a partial response. DP22 had no effect of doxycycline-regulated TREK-M expression in its normalized seizure frequency. 
A

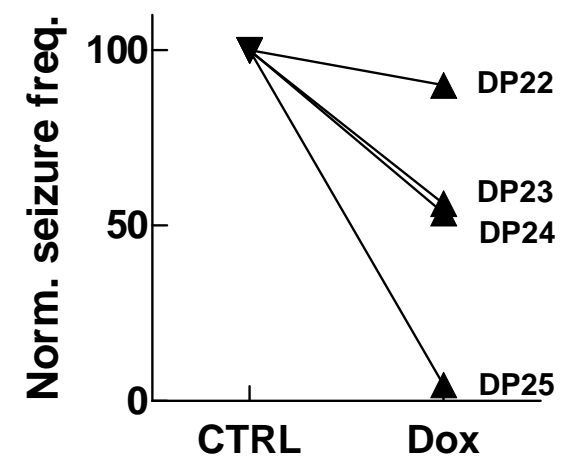

B

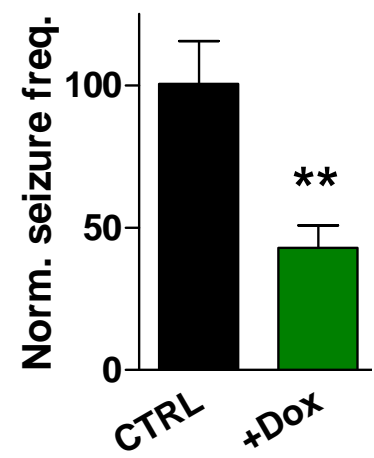

Figure 14: Effect of AAV5-SmonCeiT on normalized seizure frequency of rats

A: The normalized seizure frequency of DP25, DP24, DP23 and DP22 has been shown.

B: The normalized seizure frequency significantly decreased when was added to drinking water compared to baseline seizure frequency; $* * \mathrm{p}<.005$ 


\section{4. mCherry expression was seen in the EC and DG of DP25 6 months after injection with AA V5-SmonCeiT}

In order to study the expression of TREK-M in the brain of DP25, who was the best responder of these 4 rats, the rat was perfused and its brain was sliced. mCherry expression regulated by doxycycline was seen in the EC layer IV and V and in the subiculum (Fig 15). The expression of mCherry is an accurate reporter of TREK-M since both mCherry and TREK-M are transcribed from the same mRNA. 


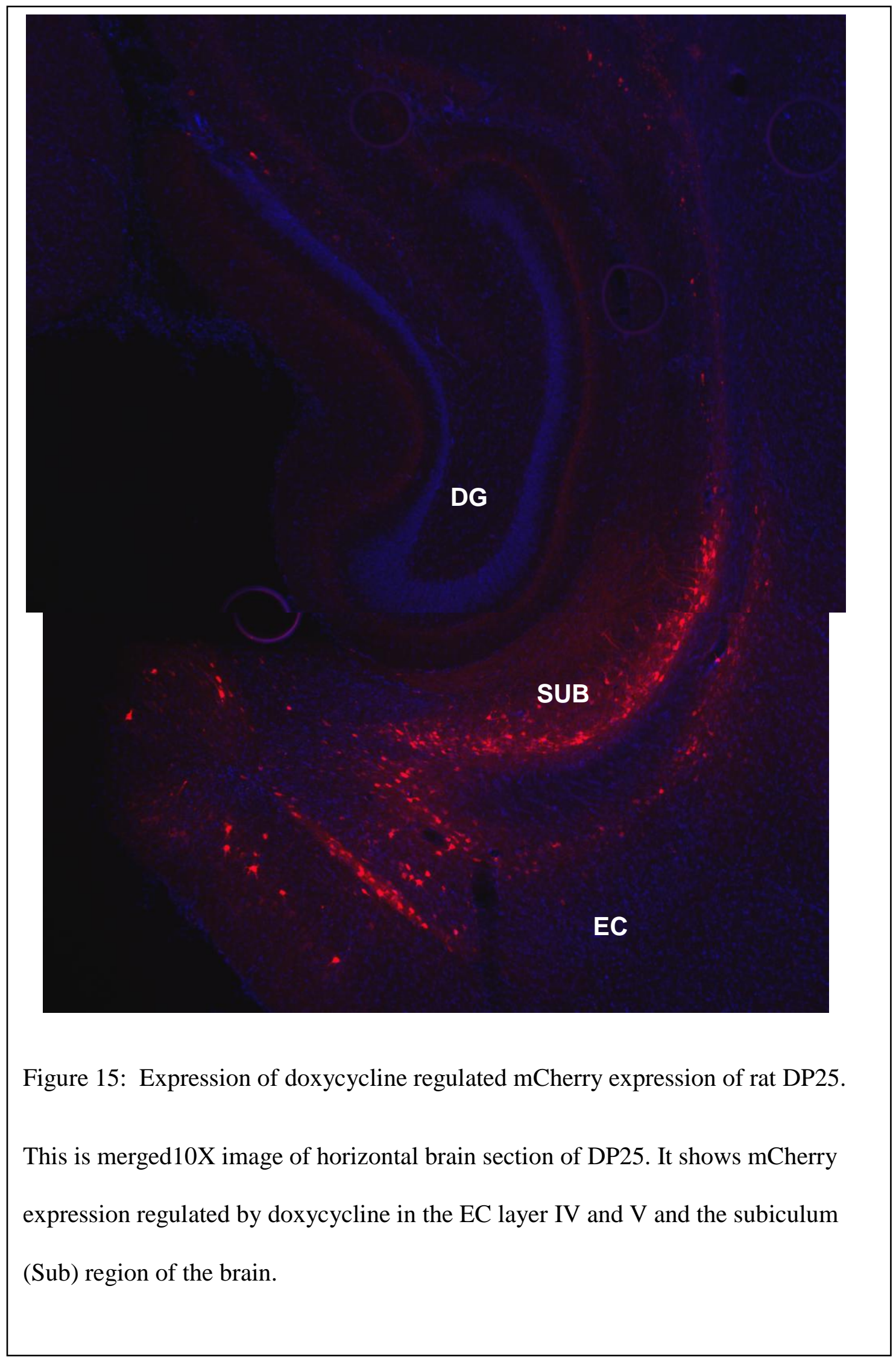




\section{DISCUSSION}

The goal of this study was to test gene therapy treatment in an animal model of temporal lobe epilepsy using drug regulated expression of the mutated potassium leak channel TREK-M. Recombinant adeno-associated virus serotype 5 was used to package the doxycycline regulation cassette. Addition of drug regulation to TREK-M expression would allow turning on and off the expression of TREK-M simply by using doxycycline in its drinking water. There are three main conclusions that can be drawn from this study. First, in the absence of doxycycline there was very low expression from the AAV, and doxycycline treatment caused an expression as measured by mCherry fluorescence. Second, doxycycline regulated expression of TREK-M significantly reduced normalized seizure frequency of chronic epileptic rats. Third, mCherry expression was found in the EC and subiculum region in chronic epileptic rats 6 months after virus injection, confirming previous studies showing long term expression (> 8 years) after AAV injection (Hadaczek, Eberling et al.).

The first Dox-regulated AAV was developed by Manfresson et al (Manfredsson, Burger et al. 2009). Their system used the "regular" tetracycline repressor that is turned off by Dox. Their seminal studies provided a template for our Dox-on design, and established that doxycycline delivered in the diet regulated expression of glial cell line derived neurotrophic factor (GDNF) in the substantia nigra. In our lab we have developed the first Dox-ON AAV capable of delivering 2 genes, mCherry and TREK-M, from the same mRNA. mCherry serves as the reporter gene of the expression of TREK-M (Appendix 4). Robust mCherry expression was seen in the EC region of the brain of chronic epileptic rats after addition of doxycycline to the drinking water. The mCherry expression was 39- 
fold higher compared to the rat drinking normal water. There was only low expression of mCherry in the absence of doxycycline. Doxycycline is approved by FDA for human use, has a serum life of 14-22 hours and enters the brain (Stieger, Belbellaa et al. 2009). We have used $200 \mu \mathrm{g} / \mathrm{ml}$ of doxycycline in the drinking water (Bohl, Salvetti et al. 1998). The volume distribution of doxycycline measured in pigs is 0.5 liters $/ \mathrm{kg}$ (Riond and Riviere 1990). Assuming a similar distribution in rats, the approximate volume distribution of a 250 grams rat would be 0.125 liters. Based on typical water consumption in a 24 hour period, the initial concentration of doxycycline in the rat would reach 40 $\mu \mathrm{g} / \mathrm{ml}$. Chronic dosing likely achieves even higher concentrations as observed in man (Newton et al., 2005). Based on in vitro studies performed by Dr. Iuliia Vitko (Appendix 4), these concentrations would maximally stimulate the doxycycline cassette to express mCherry and TREK-M. Therefore, the amount of water actually drunk by each rat should not affect the results.

The effect of doxycycline regulated TREK-M expression in the brain of chronic epileptic rats was very encouraging. This experiment was very challenging due to its long duration accompanied with many rats losing headsets during the experiments. As a result, starting with ten rats, we ended up with only 4 rats to the end of the experiment. Four rats died the next day after pilocarpine injection due to status epilepticus. Out of the remaining six rats, one never had seizures during the whole phase of video monitoring or EEG recording while the other one lost its headset during the first wash-out phase. DP25 was the best responder who showed a significant decrease in seizure frequency during the second doxycycline dose and baseline period and between second wash-out and third doxycycline period. DP23 and DP24 showed partial responses, whereas DP22 was a 
complete non-responder. The normalized seizure frequency of these rats was significantly decreased when they had doxycycline in their diet. In order to test the expression of the doxycycline regulated mCherry expression in the brain, DP25 was perfused and its brain was horizontally sliced. Robust expression of mCherry was seen in the EC and subiculum area of the brain of this rat after 6 months of virus injection. Unfortunately, I left the brain of DP24 in paraformaldehyde for a month after perfusion, which I now know results in irreversible degradation of the proteins in the sample. DP22 and DP23 were not perfused, so the question why DP25 responded so well to the treatment versus DP22 cannot be answered at this point. However, we had a very interesting observation from the brain slices of DP25. In addition to robust expression of mCherry in the EC and subiculum, the dentate granule cell layer of this rat showed extensive dispersion and ectopic growth. Indeed, in the most ventral slices we observed duplication of the inferior blade of the DG (Fig 16A, 16B). Dentate granule cells undergo neurogenesis leading to dispersion due to seizures in both human temporal lobe epilepsy and rodent models of epilepsy (Parent, Timothy et al. 1997). It can be assumed that this dispersion and bifurcation of the DG layer in this rat was caused by seizures. The ability of doxycyclineregulated expression of TREK-M to suppress seizures in this rat may have been due to AAV infecting EC cells that developed projections into the new DG regions (see dense innervation surrounding entire DG layers). These studies confirm our initial hypothesis that the EC is an excellent target for AAV injection, therefore, we will inject virus in the EC for all our future experiments. In conclusion, doxycycline-regulated expression of TREK-M appears to be a promising approach to treat pharmacoresistant focal epilepsies. This study shows that doxycycline regulated expression of TREK-M in vivo with very 
little background expression. Importantly, it had a significant effect on decreasing seizure frequency in three of four rats. Refinements in injection strategies, promoter selection, AAV serotype, and expression of alternative genes-of-interest, may lead to gene therapies that someday could be used in patients with drug resistant focal epilepsies. 


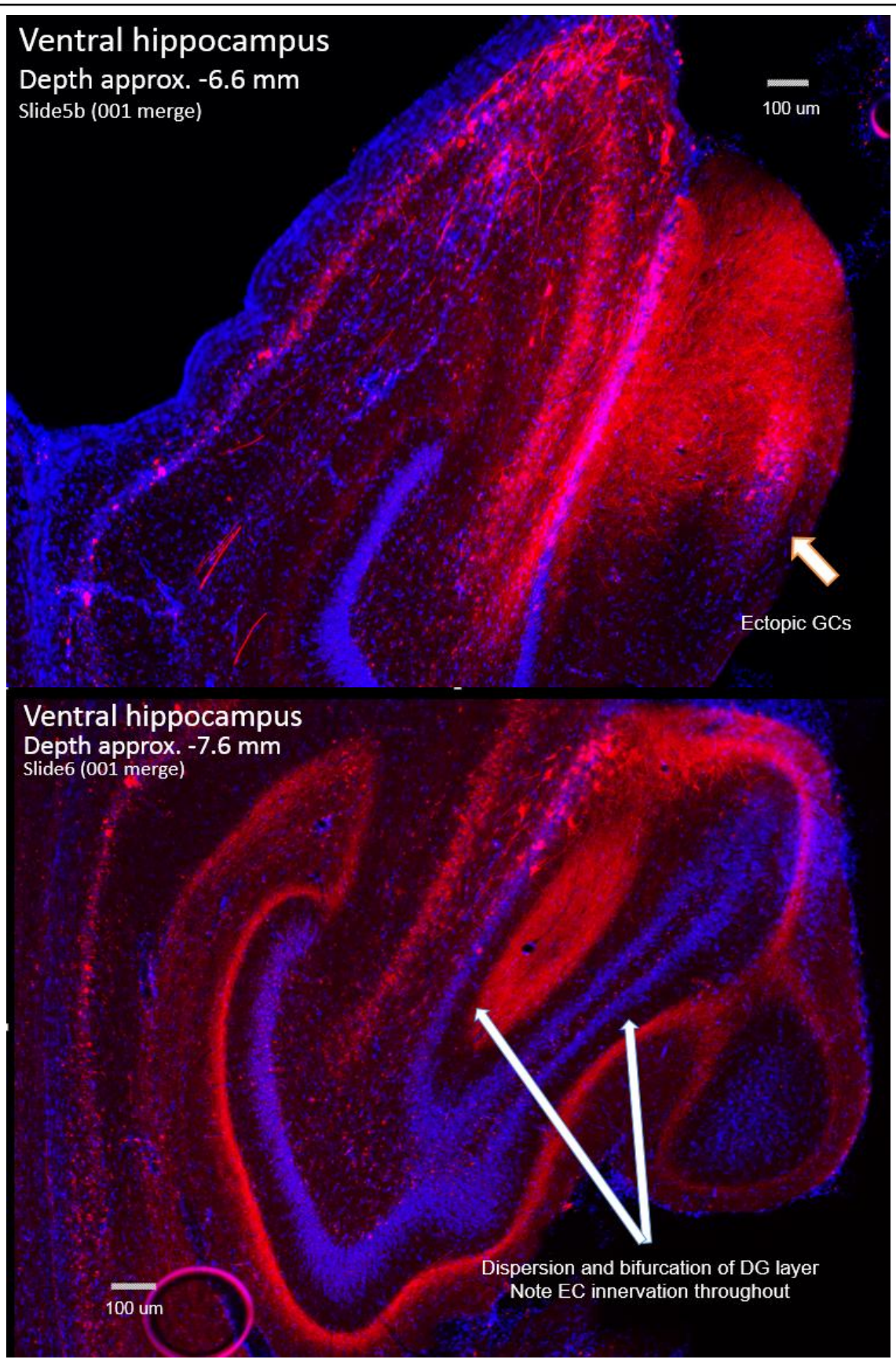

Figure 16: Altered structure of the dentate granule cell layer in DP25

A: 10X image of hippocampus of DP25 6 months after AAV5-SmonCeiT virus injection. Ectopic growth of granule cells is noted with an arrow. The inferior blade of DG appears to have dispersed, then split into two. B: 10X image of hippocampus at depth of $-7.6 \mathrm{~mm}$ from bregma. Clear bifurcation of DG layer is seen in this image. 


\section{CHAPTER 4 - Summary and future directions}

\section{Summary}

The overall focus of this study was to find an alternative gene therapy treatment for recurrent focal seizures.

1. To validate the tools required for in vivo gene therapy studies

i) Choose and validate GABRA4 promoter for driving the gene of choice for ameliorating SE

ii) Validate the site of brain regions to inject viral particles.

2. To investigate the role of pre injection of scAAV5-TREK-M particles on acute seizures in lithium/pilocarpine rat model of epilepsy

i. Identify the effect of AAV5-TREK-M viral particles on the behavior stage score BSS of epileptic rats

ii. Identify the effect of our viral particles on the electrographic score of epileptic rats

iii. Study neuronal death in epileptic versus control rats

3. To investigate the role of doxycycline-regulated expression of TREK-M on chronic seizures in lithium/pilocarpine rat model of epilepsy

i. To validate doxycycline regulated expression of mCherry and TREK-M in vivo

ii. To investigate the effect of TREK-M expression regulated by doxycycline on chronic seizures in lithium/pilocarpine rat model of epilepsy 
The results of chapter 2 and 3 of this thesis have addressed these issues.

The potential for TREK-M to silence hyper-excitable neurons was validated in vitro by the former post doctoral scientist in our lab, Veit-Simon Eckle, and included in the Epilepsia paper describing our experiments in acute SE (Dey, Eckle et al. 2013). The results from his TREK-M in vitro validation experiments are discussed very briefly (see Appendix Fig 1, Fig 2 and Fig 3). He validated that TREK-M is no longer regulated by intracellular acidosis and second messenger cascade systems (Appendix Fig 1). Also he tested that TREK-M is capable of increasing $\mathrm{K}+$ leak currents, lower membrane potential and decrease input resistance (Appendix Fig 2). Cultured hippocampal neurons were transfected with childhood absence epilepsy mutant of $\mathrm{Ca}_{\mathrm{v}} 3.2$ T-type calcium channels (C456S) in order to make them hyper-excitable (Eckle, Shcheglovitov et al. 2013). Hippocampal neurons transfected with C456S plus TREK-M plasmids fired significantly less action potentials than C456S transfected neurons suggesting TREK-M's ability to limit firing (Appendix Fig 3). Resting membrane potentials of TREK-M co-transfected hippocampal neurons were also found to be significantly hyperpolarized compared to untransfected and C456S--transfected neurons (Appendix Fig 3).

A suitable promoter is required in order to drive the expression of TREK-M in rodent brain. GABRA4 promoter emerged as our top choice to drive the expression of Trek $\mathrm{M}$ in vivo.

The transfection experiments revealed the capability of this promoter to drive fluorescent protein expression only in ND7/23 neuroblastoma cell line and not in non neuronal HEK293 cells confirming the fact that it exhibits neuron specific expression. Its small size of 
542 bp made it possible to package into a self-complementary AAV vector. Studies have also shown the activity of this promoter being epileptic activity dependent.

Initial experiments in our lab showed that the original 542 bp long GABRA4 promoter to be a very weak promoter. In order to increase its strength we engineered by adding CMV enhancer sequence at its $5^{\prime}$ ' end and CMV minimum sequence at its $3^{\prime}$ end. CMV minimum sequence contains a TATA box and TFII $\beta$ binding site which results in increased expression of gene situated downstream of this sequence (Loew, Heinz et al.). We also wanted to make sure that in spite of all these modifications, it retained its neuronal specificity. ND7/23 neuroblastoma cells transfected with GABRA4 promoter driving yellow fluorescent protein showed robust yellow fluorescence while nonneuronal HEK-293 cells transfected with the same plasmid construct did not show any fluorescent positive cells.

The second sub aim under specific aim 1 was to choose the regions to inject TREK-M viral particles in brain so that to get maximum transduction of neurons. EC and CA3 were chosen to be the sites of AAV-TREK-M viral particles for the experiments in order to disrupt the epileptic circuit at maximum points. Bilateral AAV-GFP viral particle injections into $\mathrm{CA} 3$ and $\mathrm{EC}$ of rodent brain showed robust green fluorescence in hippocampus. GFP fluorescence has been detected in CA3 pyramidal neurons, dentate granule layer and subicular neurons. Robust fluorescence was also seen in layers II, IV and $\mathrm{V}$ of EC.

The second aim was to focus on in vivo studies and look at effect of TREK-M on SE and neuronal death. The first sub aim of the second specific aim was to find out whether there 
is any change in behavioral seizures by AAV-TREK-M rats in the first two hours after pilocarpine injection. No difference in the BSS score was observed between experimental versus control rats. Rats pre-injected with AAV-TREK-M spent significantly less time in SE compared to control rats. Also significant less neuronal death was observed in EC, CA3, CA1 regions of rats injected with TREK-M viral particles compared to controls. These findings suggest that TREK-M definitely can emerge as an alternative gene therapy choice for SE patients. These studies also pave the way for TREK-M's capability of serving as chosen gene for chronic TLE patients. We also tried to study the effect of AAV-GABRA4-TREK-M virus on chronic seizures, but failed largely due to technical problems. In order to study the effect of scAAV5-GABRA4-TREK-M on chronic seizures, each rat underwent two surgeries. The first surgery was headset implantation in order to record the baseline seizure frequency. The second surgery was virus injection, which was done through the cranioplast of the headset. This was very challenging because the bregma or lambda was not visible through the headset and even calculating the exact EC location from the ear bars it cannot be said for sure if the exact EC spot was injected. In some rats, they had blood loss during this surgery and died. As a result of these problems, we decided to study the effect of this virus in acute seizures and status epilepticus in pilocarpine rat model of epilepsy.

The third aim of this study was to investigate the effect of doxycycline regulated expression of TREK-M on chronic seizures in lithium pilocarpine model of seizures in rats. Under this aim, there were mainly two sub aims. We validated the effect of doxycycline regulated expression of TREK-M in chronic epileptic rats. Our results have shown that doxycycline regulated expression of TREK-M in chronic epileptic rats 
showed very little expression in absence of doxycycline. Doxycycline regulated expression of TREK-M showed very promising results in treating chronic seizures in lithium pilocarpine model of epilepsy. One out of four rats was completely cured by this treatment whereas two of them showed partial response. The normalized seizure frequency of these four rats was significantly decreased in presence of doxycycline in drinking water. The chronic experiments were challenging since we ended up with only four rats starting from ten. Losing headsets was a common problem during these long experiments and we are trying to improve that by switching to new adhesives and cranioplast.

The in vitro validation of SmonCeiT construct was done by Dr. Vitko in our lab (see Appendix figure 4). The dose response curve of doxycycline was done in ND7/23 cells transfected with SmonCeiT construct. This vector uses the synapsin promoter to drive expression of the doxycycline cassette (Appendix figure 4 A). Application of 10-30 $\mu \mathrm{g} / \mathrm{ml}$ of doxycycline in the culture medium caused a 100-fold increase in expression of mCherry. The TREK-M currents were validated in SmonCeiT transfected ND7/23 cells (Appendix figure 4B).

Developing a gene therapy to treat focal seizures in epilepsy is a difficult task to achieve. This study showing the ability to control seizures with TREK-M looks promising and can be applied for treating patients who are suffering from recurrent, pharmacoresistant seizures with a focal origin. Future studies are required to address many outstanding issues, such as: optimal dosing strategy, immune responses, and the possible effect of TREK-M on sleep and learning. 


\section{Future Directions}

This thesis suggests immense possibilities of future work.

\section{Investigate the functional property of AAV-TREK-M infected neurons}

The rats pre injected with TREK-M viral particles spent almost 50\% less time in status epilepticus after pilocarpine injection compared to controls. This clearly suggests that TREK-M incorporation reduced the hyper excitation of neurons and as observed in vitro (Appendix Fig 1 and 2); this was due to a decrease in membrane potential of the neurons that prevented action potential generation. In order to confirm it, slice electrophysiological recordings could be done. A different viral construct is needed for this experiment. The viral construct should have a fluorescent protein reporter gene in addition to TREK-M in order to visualize the neurons while patching. The viral particles should be allowed to express in rats for at least 2 weeks prior to lithium/pilocarpine injection. Spontaneous firing, resting membrane potential should be measured from neurons which show fluorescence. Control rats should be injected with AAV driving fluorescent protein.

\section{AAV serotype 1 to drive TREK-M gene in rat brain}

AAV2 is the most commonly used serotype among AAV vectors. The serotype that was chosen for this thesis was serotype 5. Studies have shown that the transduction of CA1CA3 regions of brain by serotype 5 was significantly higher than the common used AAV serotype 2 (Burger, Gorbatyuk et al. 2004). More and more studies are coming up with different AAV serotypes like AAV 1, 6, 8 and 9. Recent studies have shown that serotype 1 has more transduction capacity in hippocampus (Noe, Vaghi et al. 2010). 
AAV 1 can express high gene expression in only 4 days compared to serotypes 2 or 5 which require almost 2-4 weeks for expression (Su, Huang et al. 2006). The transduction efficiency of AAV 1 in murine brain have been found to be 13-35 fold higher than AAV2 (Wang, Wang et al. 2003). Using AAV serotype 1 to drive TREK-M could lead to higher transduction rates of neurons within 3-4 days, which could lead to an efficient gene therapy treatment for epilepsy. Also AAV-1 serotype has recently been approved for clinical use in Europe.

\section{Subiculum as new site of injection}

A big question in the field of temporal lobe epilepsy research is where do seizures begin? Knowing the site of seizure generation would be beneficial because injecting gene of choice in that particular site would effectively stop seizure generation as well as propagation. Studies from animal models of epilepsy have revealed multiple sites where seizures begin such as amygdala (Bertram 1997), midline dorsal thalamus (Bertram, Mangan et al. 2001), and parts of hippocampal formation (Lévesque, Salami et al. 2012). There have always been criticism about the seizure initiation site in animal epileptic models may be different from epileptic patients. In TLE patients different studies have first recorded seizures from either hippocampal formation, amygdale, temporal lobe or neocortex (Toyoda, Bower et al. 2013). Recent studies have shown that the seizures appear first at the ventral subiculum (Toyoda, Bower et al. 2013). Early studies have already shown that seizures spread through the ventral subiculum (Kliot and Poletti 1979). Furthermore, it has been observed in TLE patients that seizures originated in this particular area (Huberfeld, de la Prida et al. 2011). According to these studies the ventral subiculum serves as a common area between rodent model of epilepsy and TLE patients 
as the site for early seizure generation. Thus, the ventral subiculum would be a good site of injection of AAV particles driving TREK-M to study its effect on chronic epilepsy in rodents. Injecting TREK-M in this area would dampen the hyper-excitable neurons at the seizure origin site and stop the spread of seizures to other brain regions.

\section{Other approaches to combat seizures}

Epilepsy results due to a disturbance in the balance between the excitation and inhibition in the brain. There are two ways to combat the increased hyperexcitability of neurons. Dampening the excitation will restore the balance and stop seizures. We applied this approach by delivering TREK-M into epileptic rat brain via adeno-associated virus. The other approach will be to increase the inhibitory response which can counterbalance the increased excitation and stop seizures.

The neuropeptide somatostatin (Farooq, Ali-Musstjab-Akber-Shah Eqani et al.) has been found to play important role in epilepsy (Tallent and Qiu 2008). Kainate induced epileptic rats have less SST positive neurons in the inner part of hilus (Buckmaster and Dudek 1997). SST neurons synapse with distal dendrites of granule cells and regulate the excitatory input into the hippocampus (Tallent and Qiu 2008). SST modulates synaptic plasticity by preventing long-term potentiation generation (LTP) (Baratta, Lamp et al. 2002). It inhibits post-synaptic $\mathrm{N}$ type $\mathrm{Ca}^{2+}$ channels which depolarize dendrites to induce LTP (Baratta, Lamp et al. 2002). The expression of the neuropeptide SST in the dentate gyrus could control neuronal excitability in hippocampus. It would be interesting to package SST gene and its receptor in an adeno-associated virus release in 
hippocampus (Tallent and Qiu 2008) so this virus would express SST and the receptor in the hippocampus and control seizures in epileptic rats.

Adenosine is an endogenous anticonvulsant which exhibits inhibitory action in the brain (Hargus, Jennings et al. 2012). There are four adenosine receptors which have been identified so far. They are $A_{1}, A_{2 A}, A_{2 B}$ and $A_{3}, A_{1}$ and $A_{2 A}$ receptors are mostly found in CNS. $A_{1}$ receptors play an important role in neuroprotection (Wardas 2002). The mode of action of adenosine is as follows. It binds to $A_{1}$ receptors which activate the inhibitory $G$ protein $(\mathrm{Gi})$ which in turn causes the activation of outwardly rectifying $\mathrm{K}^{+}$channels. The post-synaptic neuron becomes hyperpolarized because of this opening of potassium channels (Trussell and Jackson 1985). The calcium entry via $\mathrm{Ca}_{\mathrm{v}} 2$ channels is also inhibited in the presynaptic neuron because of Gi activation by adenosine binding to the receptor (Scholz and Miller 1991). Study shows that the antiseizure effect by ketogenic diets is mediated also by A1 receptors (Masino, Li et al. 2011). Studies have shown increased amount of adenosine due to epileptic seizures in animal models as well as human patients (During and Spencer 1992);(Daval and Werck 1991). Adenosine (500nM-100uM) application on the hippocampal slices from chronic epileptic rats reduced the action potential frequency and evoked action potential by synapses (Hargus, Jennings et al. 2012). Adenosine is converted in hippocampal slices from AMP in less than a second and this step is also the rate limiting one (Dunwiddie and Masino 2001). Extracellular release of ATP aggravates seizures (Kumaria, Tolias et al. 2008). The balance from overexcitation to normal firing can be tipped by conversion of ATP to adenosine. Local adenosine concentration in hippocampus can be increased by 
ectonucleotidases expression which in turn will activate A1 signaling, leading to reduced neuronal firing and seizures. 


\section{References}

Adil Harroud, A. B., 2 Alexander G. Weil,2 and Dang Khoa Nguyen1 (2012). "Temporal Lobe Epilepsy Surgery Failures: A Review." Epilepsy Research and Treatment 2012.

Anderson P, M. R., Amaral D, Bliss T, O'Keefe J (2007). The Hippocampus Book. New York, Oxford University Press,Inc.

Aronica, E., B. Yankaya, et al. (2001). "Induction of neonatal sodium channel II and III $\alpha$-isoform mRNAs in neurons and microglia after status epilepticus in the rat hippocampus." European Journal of Neuroscience 13(6): 1261-1266.

Babb, T., W. Kupfer, et al. (1991). "Synaptic reorganization by mossy fibers in human epileptic fascia dentata." Neuroscience 42(2): 351-363.

Babb, T. L., W. J. Brown, et al. (1984). "Temporal-Lobe Volumetric Cell Densities in Temporal-Lobe Epilepsy." Epilepsia 25(6): 729-740.

Baratta, M. V., T. Lamp, et al. (2002). "Somatostatin depresses long-term potentiation and $\mathrm{Ca} 2+$ signaling in mouse dentate gyrus." Journal of neurophysiology 88(6): 30783086.

Bartolomei, F., M. Khalil, et al. (2005). "Entorhinal cortex involvement in human mesial temporal lobe epilepsy: an electrophysiologic and volumetric study." Epilepsia 46(5): 677-687.

Bayliss, D. A. and P. Q. Barrett (2008). "Emerging roles for two-pore-domain potassium channels and their potential therapeutic impact." Trends Pharmacol Sci 29(11): 566-575.

Bear, J., N. B. Fountain, et al. (1996). "Responses of the superficial entorhinal cortex in vitro in slices from naive and chronically epileptic rats." J Neurophysiol 76(5): 29282940.

Bell, B., J. J. Lin, et al. "The neurobiology of cognitive disorders in temporal lobe epilepsy." Nat Rev Neurol 7(3): 154-164.

Bell, B., J. J. Lin, et al. (2011). "The neurobiology of cognitive disorders in temporal lobe epilepsy." Nature Reviews Neurology 7(3): 154-164. 
Benkovic, S. A., J. P. O'Callaghan, et al. (2004). "Sensitive indicators of injury reveal hippocampal damage in C57BL/6J mice treated with kainic acid in the absence of tonicclonic seizures." Brain Res 1024(1-2): 59-76.

Bertram, E. H. (1997). "Functional anatomy of spontaneous seizures in a rat model of limbic epilepsy." Epilepsia 38(1): 95-105.

Bertram, E. H., P. S. Mangan, et al. (2001). "The midline thalamus: alterations and a potential role in limbic epilepsy." Epilepsia 42(8): 967-978.

Blakemore, A., S. H. Wang, et al. (2008). "Model-based insulin sensitivity as a sepsis diagnostic in critical care." J Diabetes Sci Technol 2(3): 468-477.

Blumenfeld, H., A. Lampert, et al. (2009). "Role of hippocampal sodium channel Nav1. 6 in kindling epileptogenesis." Epilepsia 50(1): 44-55.

Bohl, D., A. Salvetti, et al. (1998). "Control of erythropoietin delivery by doxycycline in mice after intramuscular injection of adeno-associated vector." Blood 92(5): 1512-1517.

Brodie, M. J., S. C. Schachter, et al. (2012). Fast facts: epilepsy, Health Press.

Brooks-Kayal, A. R., M. D. Shumate, et al. (1998). "Selective changes in single cell $\operatorname{GABA}(\mathrm{A})$ receptor subunit expression and function in temporal lobe epilepsy [see comments] [published erratum appears in Nat Med 1999 May;5(5):590]." Nature Medicine 4(10): 1166-1172.

Brophy, G. M., R. Bell, et al. (2012). "Guidelines for the Evaluation and Management of Status Epilepticus." Neurocritical Care 17(1): 3-23.

Bruton, C. J. (1988). The neuropathology of temporal lobe epilepsy, Oxford University Press New York.

Buckmaster, P. S. (2004). "Laboratory animal models of temporal lobe epilepsy." Comp Med 54(5): 473-485. 
Buckmaster, P. S. and F. E. Dudek (1997). "Network properties of the dentate gyrus in epileptic rats with hilar neuron loss and granule cell axon reorganization." J Neurophysiol 77(5): 2685-2696.

Buckmaster, P. S. and F. E. Dudek (1997). "Neuron loss, granule cell axon reorganization, and functional changes in the dentate gyrus of epileptic kainate-treated rats." Journal of Comparative Neurology 385(3): 385-404.

Buckmaster, P. S., G. F. Zhang, et al. (2002). "Axon sprouting in a model of temporal lobe epilepsy creates a predominantly excitatory feedback circuit." J Neurosci 22(15): 6650-6658.

Burger, C., O. S. Gorbatyuk, et al. (2004). "Recombinant AAV viral vectors pseudotyped with viral capsids from serotypes 1,2, and 5 display differential efficiency and cell tropism after delivery to different regions of the central nervous system." Mol Ther 10(2): 302-317.

Carter, B. J. (2004). "Adeno-associated virus and the development of adeno-associated virus vectors: a historical perspective." Mol Ther 10(6): 981-989.

Carter, B. J. (2005). "Adeno-associated virus vectors in clinical trials." Hum Gene Ther 16(5): 541-550.

Chevaleyre, V. and S. A. Siegelbaum (2010). "Strong CA2 pyramidal neuron synapses define a powerful disynaptic cortico-hippocampal loop." Neuron 66(4): 560-572.

Corkin, S., D. G. Amaral, et al. (1997). "HM's medial temporal lobe lesion: findings from magnetic resonance imaging." The Journal of Neuroscience 17(10): 3964-3979.

Curia, G., D. Longo, et al. (2008). "The pilocarpine model of temporal lobe epilepsy." $\underline{\mathbf{J}}$ Neurosci Methods 172(2): 143-157.

Daval, J.-L. and M.-C. Werck (1991). "Autoradiographic changes in brain adenosine A< sub> $1</$ sub $>$ receptors and their coupling to $G$ proteins following seizures in the developing rat." Developmental brain research 59(2): 237-247.

DeLorenzo, R. J., W. A. Hauser, et al. (1996). "A prospective, population-based epidemiologic study of status epilepticus in Richmond, Virginia." Neurology 46(4): 1029-1035. 
Detry, O., A. De Roover, et al. (2007). "[Recent advances in liver surgery and transplantation]." Rev Med Liege 62(5-6): 310-316.

Dey, D., Eckle, V.-S., et al. (2013) "A potassium leak channel silences hyperactive neurons and ameliorates status epilepticus." Epilepsia in press.

Du, F., T. Eid, et al. (1995). "Preferential neuronal loss in layer III of the medial entorhinal cortex in rat models of temporal lobe epilepsy." The Journal of neuroscience 15(10): 6301-6313.

Du, F., W. O. Whetsell Jr, et al. (1993). "Preferential neuronal loss in layer III of the entorhinal cortex in patients with temporal lobe epilepsy." Epilepsy research 16(3): 223233.

Dunwiddie, T. V. and S. A. Masino (2001). "The role and regulation of adenosine in the central nervous system." Annual review of neuroscience 24(1): 31-55.

During, M. J. and D. D. Spencer (1992). "Adenosine: a potential mediator of seizure arrest and postictal refractoriness." Annals of neurology 32(5): 618-624.

Eckle, V.-S., Shcheglovitov, A., et al. (2013) "Mechanisms by which CACNA1H

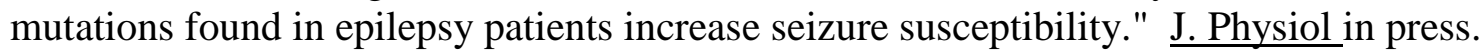

Engel, J. (2013). Seizures and epilepsy, Oxford University Press.

Engel, J., T. A. Pedley, et al., Eds. (2008). Epilepsy: A comprehensive textbook Philadelphia, Lippincott Williams \& Wilkins.

Enyedi, P. and G. Czirjak "Molecular background of leak K+ currents: two-pore domain potassium channels." Physiol Rev 90(2): 559-605.

Farooq, S., S. Ali-Musstjab-Akber-Shah Eqani, et al. "Occurrence, finger printing and ecological risk assessment of polycyclic aromatic hydrocarbons (PAHs) in the Chenab River, Pakistan." J Environ Monit. 
Fisher, R. S., W. v. E. Boas, et al. (2005). "Epileptic seizures and epilepsy: definitions proposed by the International League Against Epilepsy (ILAE) and the International Bureau for Epilepsy (IBE)." Epilepsia 46(4): 470-472.

French, J. A., P. D. Williamson, et al. (1993). "Characteristics of medial temporal lobe epilepsy: I. Results of history and physical examination." Ann Neurol 34(6): 774-780.

Goldstein, S. A., D. A. Bayliss, et al. (2005). "International Union of Pharmacology. LV. Nomenclature and molecular relationships of two-P potassium channels." $\underline{\text { Pharmacol Rev }}$ 57(4): 527-540.

Goodkin, H. P. and J. Kapur (2009). "The impact of diazepam's discovery on the treatment and understanding of status epilepticus." Epilepsia 50(9): 2011-2018.

Gossen, M., S. Freundlieb, et al. (1995). "Transcriptional activation by tetracyclines in mammalian cells." Science 268(5218): 1766-1769.

Gould, D. J. and P. Favorov (2003). "Vectors for the treatment of autoimmune disease." Gene Therapy 10(10): 912-927.

Greene, J. G. and J. T. Greenamyre (1996). "Bioenergetics and glutamate excitotoxicity." Prog Neurobiol 48(6): 613-634.

Gunthorpe, M. J., C. H. Large, et al. (2012). "The mechanism of action of retigabine (ezogabine), a first-in-class $\mathrm{K}+$ channel opener for the treatment of epilepsy." Epilepsia 53(3): 412-424.

Haberman, R., H. Criswell, et al. (2002). "Therapeutic liabilities of in vivo viral vector tropism: adeno-associated virus vectors, NMDAR1 antisense, and focal seizure sensitivity." Mol Ther 6(4): 495-500.

Hadaczek, P., J. L. Eberling, et al. "Eight years of clinical improvement in MPTPlesioned primates after gene therapy with AAV2-hAADC." Mol Ther 18(8): 1458-1461.

Hamilton, S. E., M. D. Loose, et al. (1997). "Disruption of the m1 receptor gene ablates muscarinic receptor-dependent $\mathrm{M}$ current regulation and seizure activity in mice." Proc Natl Acad Sci U S A 94(24): 13311-13316. 
Hargus, N. J., C. Jennings, et al. (2012). "Enhanced actions of adenosine in medial entorhinal cortex layer II stellate neurons in temporal lobe epilepsy are mediated via A1-receptor activation." Epilepsia 53(1): 168-176.

Heilbronn, R. and S. Weger "Viral vectors for gene transfer: current status of gene therapeutics." Handb Exp Pharmacol(197): 143-170.

Hellier, J. L., P. R. Patrylo, et al. (1998). "Recurrent spontaneous motor seizures after repeated low-dose systemic treatment with kainate: assessment of a rat model of temporal lobe epilepsy." Epilepsy Res 31(1): 73-84.

Hesdorffer, D. C., G. Logroscino, et al. (1998). "Incidence of status epilepticus in Rochester, Minnesota, 1965-1984." Neurology 50(3): 735-741.

Hille, B. (2001). Ionic channels of excitable membranes. Sunderland, Sinauer Associates, Inc.

Honchar, M. P., J. W. Olney, et al. (1983). "Systemic cholinergic agents induce seizures and brain damage in lithium-treated rats." Science 220(4594): 323-325.

Honore, E. (2007). "The neuronal background K2P channels: focus on TREK1." Nat Rev Neurosci 8(4): 251-261.

Honore, E., F. Maingret, et al. (2002). "An intracellular proton sensor commands lipidand mechano-gating of the K(+) channel TREK-1." EMBO J 21(12): 2968-2976.

Honore, E., F. Maingret, et al. (2002). "An intracellular proton sensor commands lipidand mechano-gating of the $\mathrm{K}^{+}$channel TREK-1." EMBO J 21(12): 2968-2976.

Honore, E., A. J. Patel, et al. (2006). "Desensitization of mechano-gated K2P channels." Proc Natl Acad Sci U S A 103(18): 6859-6864.

Horton, R. M., S. N. Ho, et al. (1993). "Gene splicing by overlap extension." Methods Enzymol 217: 270-279.

Houser, C. R. (1990). "Granule cell dispersion in the dentate gyrus of humans with temporal lobe epilepsy." Brain research 535(2): 195-204. 
Huberfeld, G., L. M. de la Prida, et al. (2011). "Glutamatergic pre-ictal discharges emerge at the transition to seizure in human epilepsy." Nature neuroscience 14(5): 627634.

Jones, M. W. and T. J. McHugh "Updating hippocampal representations: CA2 joins the circuit." Trends Neurosci 34(10): 526-535.

Jope, R. S., R. A. Morrisett, et al. (1986). "Characterization of Lithium Potentiation of Pilocarpine-Induced Status Epilepticus in Rats." Experimental Neurology 91(3): 471-480.

Joshi, S. and J. Kapur (2012). "GABAA Receptor Plasticity During Status Epilepticus."

Jutila, L., A. Ylinen, et al. (2001). "MR volumetry of the entorhinal, perirhinal, and temporopolar cortices in drug-refractory temporal lobe epilepsy." AJNR Am J Neuroradiol 22(8): 1490-1501.

Kapur, J. and R. L. Macdonald (1997). "Rapid seizure-induced reduction of benzodiazepine and $\mathrm{Zn} 2+$ sensitivity of hippocampal dentate granule cell GABAA receptors." J Neurosci 17(19): 7532-7540.

Kapur, J. and R. L. Macdonald (1997). "Rapid seizure-induced reduction of benzodiazepine and $\mathrm{Zn} 2+$ sensitivity of hippocampal dentate granule cell GABAA receptors." The Journal of neuroscience 17(19): 7532-7540.

Kellinghaus, C., S. Berning, et al. (2011). "Intravenous lacosamide for treatment of status epilepticus." Acta neurologica Scandinavica 123(2): 137-141.

Ketchum, K. A., W. J. Joiner, et al. (1995). "A new family of outwardly rectifying potassium channel proteins with two pore domains in tandem." Nature 376(6542): 690695.

Ketelaars, S., J. Gorter, et al. (2001). "Sodium currents in isolated rat CA1 pyramidal and dentate granule neurones in the post-status epilepticus model of epilepsy." Neuroscience 105(1): 109-120.

Kliot, M. and C. E. Poletti (1979). "Hippocampal afterdischarges: differential spread of activity shown by the [14C] deoxyglucose technique." Science 204(4393): 641-643. 
Knake, S., J. Gruener, et al. (2008). "Intravenous levetiracetam in the treatment of benzodiazepine refractory status epilepticus." J Neurol Neurosurg Psychiatry 79(5): 588589.

Kobayashi, M. and P. S. Buckmaster (2003). "Reduced inhibition of dentate granule cells in a model of temporal lobe epilepsy." J Neurosci 23(6): 2440-2452.

Kobayashi, M., X. Wen, et al. (2003). "Reduced inhibition and increased output of layer II neurons in the medial entorhinal cortex in a model of temporal lobe epilepsy." $\underline{\mathbf{J}}$ Neurosci 23(24): 8471-8479.

Kumar, S. S. and P. S. Buckmaster (2006). "Hyperexcitability, interneurons, and loss of GABAergic synapses in entorhinal cortex in a model of temporal lobe epilepsy." $\underline{\mathrm{J}}$ Neurosci 26(17): 4613-4623.

Kumaria, A., C. M. Tolias, et al. (2008). "ATP signalling in epilepsy." $\underline{\text { Purinergic }}$ signalling 4(4): 339-346.

Lemos, T. and E. A. Cavalheiro (1995). "Suppression of pilocarpine-induced status epilepticus and the late development of epilepsy in rats." Experimental brain research 102(3): 423-428.

Leppik, I. E., F. E. Dreifuss, et al. (1987). "A controlled study of progabide in partial seizures: methodology and results." Neurology 37(6): 963-968.

Lévesque, M., P. Salami, et al. (2012). "Two seizure-onset types reveal specific patterns of high-frequency oscillations in a model of temporal lobe epilepsy." The Journal of Neuroscience 32(38): 13264-13272.

Lévesque, M. F., N. Nakasato, et al. (1991). "Surgical treatment of limbic epilepsy associated with extrahippocampal lesions: the problem of dual pathology." Journal of neurosurgery 75(3): 364-370.

Li, L., F. Cendes, et al. (1999). "Surgical outcome in patients with epilepsy and dual pathology." Brain 122(5): 799-805.

Lipton, S. A. and P. A. Rosenberg (1994). "Excitatory Amino-Acids as a Final Common Pathway for Neurologic Disorders." New England Journal of Medicine 330(9): 613-622. 
Loew, R., N. Heinz, et al. "Improved Tet-responsive promoters with minimized background expression." BMC Biotechnol 10: 81.

Loscher, W. (2011). "Critical review of current animal models of seizures and epilepsy used in the discovery and development of new antiepileptic drugs." Seizure-European Journal of Epilepsy 20(5): 359-368.

Lothman, E. W., E. H. Bertram, et al. (1989). "Self-Sustaining Limbic Status Epilepticus Induced by Continuous Hippocampal-Stimulation - Electrographic and BehavioralCharacteristics." Epilepsy Research 3(2): 107-119.

Lothman, E. W., E. H. Bertram III, et al. (1991). "Functional anatomy of hippocampal seizures." Progress in neurobiology 37(1): 1-82.

Lothman, E. W. and H. B. Edward III (1993). "Epileptogenic effects of status epilepticus." Epilepsia 34(s1): S59-S70.

Lothman, E. W., D. A. Rempe, et al. (1995). "Changes in excitatory neurotransmission in the CA1 region and dentate gyrus in a chronic model of temporal lobe epilepsy." $\underline{\mathbf{J}}$ Neurophysiol 74(2): 841-848.

Lowenstein, D. H. (1998). "Status epilepticus." West J Med 168(4): 263.

Lowenstein, D. H. (1998). "Status epilepticus." Western Journal of Medicine 168(4): 263-263.

Lowenstein, D. H. (1999). "Status epilepticus: An overview of the clinical problem." Epilepsia 40: S3-S8.

Lowenstein, D. H. and B. K. Alldredge (1993). "Status epilepticus at an urban public hospital in the 1980s." Neurology 43(3 Part 1): 483-483.

Lowenstein, D. H. and B. K. Alldredge (1998). "Status epilepticus." New England Journal of Medicine 338(14): 970-976.

Lu, Y. (2004). "Recombinant adeno-associated virus as delivery vector for gene therapy A review." Stem Cells and Development 13(1): 133-145. 
Lu, Z. J., A. Pereverzev, et al. (2004). "Arrhythmia in isolated prenatal hearts after ablation of the $\mathrm{Ca}_{\mathrm{v}} 2.3$ (a1E) subunit of voltage-gated $\mathrm{Ca}^{2+}$ channels." Cell Physiol Biochem 14(1-2): 11-22.

Ma, L., L. Song, et al. (2004). "Transcriptional regulation of the mouse gene encoding the alpha-4 subunit of the GABAA receptor." J Biol Chem 279(39): 40451-40461.

Macdonald, R. L. and E. Botzolakis (2009). "GABAA receptor channels." Physiology and Pathology of chloride transporters and channels in the nervous system: From molecules to diseases: 257.

Macdonald, R. L. and K. M. Kelly (1994). "Mechanisms of action of currently prescribed and newly developed antiepileptic drugs." Epilepsia 35(Suppl 4): S41-50.

Manfredsson, F. P., C. Burger, et al. (2009). "Tight Long-term dynamic doxycycline responsive nigrostriatal GDNF using a single rAAV vector." Mol Ther 17(11): 18571867.

Masino, S. A., T. Li, et al. (2011). "A ketogenic diet suppresses seizures in mice through adenosine A1 receptors." The Journal of clinical investigation 121(7): 2679.

Mathern, G. W., T. L. Babb, et al. (1995). "Reactive synaptogenesis and neuron densities for neuropeptide Y, somatostatin, and glutamate decarboxylase immunoreactivity in the epileptogenic human fascia dentata." The Journal of neuroscience 15(5): 3990-4004.

Mazarati, A. M., R. A. Baldwin, et al. (1998). "Time-dependent decrease in the effectiveness of antiepileptic drugs during the course of self-sustaining status epilepticus." Brain research 814(1): 179-185.

McCarty, D. M. (2008). "Self-complementary AAV vectors; advances and applications." Mol Ther 16(10): 1648-1656.

McCarty, D. M. (2008). "Self-complementary AAV vectors; Advances and applications." Molecular Therapy 16(10): 1648-1656.

McCarty, D. M., P. E. Monahan, et al. (2001). "Self-complementary recombinant adenoassociated virus (scAAV) vectors promote efficient transduction independently of DNA synthesis." Gene Ther 8(16): 1248-1254. 
McCormick, D. A. and D. Contreras (2001). "On the cellular and network bases of epileptic seizures." Annu Rev Physiol 63: 815-846.

McCown, T. J. (2009). "Adeno-associated virus vector-mediated expression and constitutive secretion of galanin suppresses limbic seizure activity." Neurotherapeutics 6(2): 307-311.

McDonald, T. F., S. Pelzer, et al. (1994). "Regulation and modulation of calcium channels in cardiac, skeletal, and smooth muscle cells." Physiological Reviews 74(2): 365-507.

Meldrum, B. S. and R. W. Horton (1973). "Physiology of status epilepticus in primates." Archives of neurology 28(1): 1 .

Mizuguchi, H. and T. Hayakawa (2002). "The tet-off system is more effective than the tet-on system for regulating transgene expression in a single adenovirus vector." $\mathrm{J}$ Gene Med 4(3): 240-247.

Murbartian, J., Q. Lei, et al. (2005). "Sequential phosphorylation mediates receptor- and kinase-induced inhibition of TREK-1 background potassium channels." J Biol Chem 280(34): 30175-30184.

Nishijima, H., T. Yasunari, et al. (2009). "Improved applications of the tetracyclineregulated gene depletion system." Biosci Trends 3(5): 161-167.

Noe, F., A. H. Pool, et al. (2008). "Neuropeptide Y gene therapy decreases chronic spontaneous seizures in a rat model of temporal lobe epilepsy." Brain 131(Pt 6): 15061515.

Noe, F., V. Vaghi, et al. (2010). "Anticonvulsant effects and behavioural outcomes of rAAV serotype 1 vector-mediated neuropeptide $\mathrm{Y}$ overexpression in rat hippocampus." Gene therapy 17(5): 643-652.

Novy, J. and A. O. Rossetti (2010). "Oral pregabalin as an add-on treatment for status epilepticus." Epilepsia 51(10): 2207-2210. 
Parent, J. M., W. Y. Timothy, et al. (1997). "Dentate granule cell neurogenesis is increased by seizures and contributes to aberrant network reorganization in the adult rat hippocampus." The Journal of Neuroscience 17(10): 3727-3738.

Patel, A. J., E. Honore, et al. (1998). "A mammalian two pore domain mechano-gated Slike K+ channel." EMBO J 17(15): 4283-4290.

Paxinos, G. and C. Watson (1998). The rat brain in stereotaxic coordinates. San Diego, Academic Press.

Paxinos, G., C. R. R. Watson, et al. (1980). "Ache-Stained Horizontal Sections of the Rat-Brain in Stereotaxic Coordinates." Journal of Neuroscience Methods 3(2): 129-149.

Penaud-Budloo, M., C. Le Guiner, et al. (2008). "Adeno-associated virus vector genomes persist as episomal chromatin in primate muscle." J Virol 82(16): 7875-7885.

Penberthy, L. T., A. Towne, et al. (2005). "Estimating the economic burden of status epilepticus to the health care system." Seizure-European Journal of Epilepsy 14(1): 4651.

Perl, T. M., L. Bédard, et al. (1990). "An outbreak of toxic encephalopathy caused by eating mussels contaminated with domoic acid." New England Journal of Medicine 322(25): 1775-1780.

Poirier, J. L., R. Capek, et al. (2000). "Differential progression of dark neuron and fluorojade labelling in the rat hippocampus following pilocarpine-induced status epilepticus." Neuroscience 97(1): 59-68.

Racine, R. J. (1972). "Modification of seizure activity by electrical stimulation. II. Motor seizure." Electroencephalogr Clin Neurophysiol 32(3): 281-294.

Rakhade, S. N., B. Yao, et al. (2005). "A common pattern of persistent gene activation in human neocortical epileptic foci." Ann Neurol 58(5): 736-747.

Raol, Y. H., I. V. Lund, et al. (2006). "Enhancing GABA(A) receptor alpha 1 subunit levels in hippocampal dentate gyrus inhibits epilepsy development in an animal model of temporal lobe epilepsy." J Neurosci 26(44): 11342-11346. 
Raol, Y. H., G. Zhang, et al. (2006). "Increased GABA(A)-receptor alpha1-subunit expression in hippocampal dentate gyrus after early-life status epilepticus." Epilepsia 47(10): 1665-1673.

Renate M. Kalnins1, A. M., 5,6, Michael M. Saling3,7, Samuel F. Berkovic2,5,6, Graeme D. Jackson2,4,6, Regula S. Briellmann2,4 (2004). "Subtle microscopic abnormalities in hippocampal sclerosis do not predict clinical features of temporal lobe epilepsy." Epilepsia 45(8): 940-947.

Riban, V., H. L. Fitzsimons, et al. (2009). "Gene therapy in epilepsy." Epilepsia 50(1): 24-32.

Richichi, C., E. J. Lin, et al. (2004). "Anticonvulsant and antiepileptogenic effects mediated by adeno-associated virus vector neuropeptide $\mathrm{Y}$ expression in the rat hippocampus." J Neurosci 24(12): 3051-3059.

Riond, J. and J. Riviere (1990). "Pharmacokinetics and metabolic inertness of doxycycline in young pigs." American journal of veterinary research 51(8): 1271-1275.

Roberts, D. S., Y. H. Raol, et al. (2005). "Egr3 stimulation of GABRA4 promoter activity as a mechanism for seizure-induced up-regulation of GABA(A) receptor alpha4 subunit expression." Proc Natl Acad Sci U S A 102(33): 11894-11899.

Rossetti, A. O. and D. H. Lowenstein (2011). "Management of refractory status epilepticus in adults: still more questions than answers." The Lancet Neurology 10(10): 922-930.

Rossetti, A. O. and D. H. Lowenstein (2011). "Management of refractory status epilepticus in adults: still more questions than answers." Lancet Neurology 10(10): 922930.

Rowland, D. C., A. P. Weible, et al. (2013). "Transgenically Targeted Rabies Virus Demonstrates a Major Monosynaptic Projection from Hippocampal Area CA2 to Medial Entorhinal Layer II Neurons." The Journal of Neuroscience 33(37): 14889-14898.

Rutecki, P. A., R. G. Grossman, et al. (1989). "Electrophysiological connections between the hippocampus and entorhinal cortex in patients with complex partial seizures." $\mathrm{J}$ Neurosurg 70(5): 667-675. 
Salmenperä, T., R. Kälviäinen, et al. (2000). "Quantitative MRI volumetry of the entorhinal cortex in temporal lobe epilepsy." Seizure 9(3): 208-215.

Scharfman, H. E. (1996). "Hyperexcitability of entorhinal cortex and hippocampus after application of aminooxyacetic acid (AOAA) to layer III of the rat medial entorhinal cortex in vitro." J Neurophysiol 76(5): 2986-3001.

Schmued, L. C., C. Albertson, et al. (1997). "Fluoro-Jade: A novel fluorochrome for the sensitive and reliable histochemical localization of neuronal degeneration." Brain Research 751(1): 37-46.

Schmued, L. C., C. C. Stowers, et al. (2005). "Fluoro-Jade C results in ultra high resolution and contrast labeling of degenerating neurons." Brain Research 1035(1): 2431.

Scholz, K. and R. Miller (1991). "Analysis of adenosine actions on Ca2+ currents and synaptic transmission in cultured rat hippocampal pyramidal neurones." The Journal of physiology 435(1): 373-393.

Schramm, J., T. Kral, et al. (2001). "Surgical treatment for neocortical temporal lobe epilepsy: clinical and surgical aspects and seizure outcome." J Neurosurg 94(1): 33-42.

Segal, M. and S. Landis (1974). "Afferents to the hippocampus of the rat studied with the method of retrograde transport of horseradish peroxidase." Brain Res 78(1): 1-15.

Shorvon, S. (2006). Status epilepticus: its clinical features and treatment in children and adults, Cambridge University Press.

Siegel, A. M., H. G. Wieser, et al. (1990). "Relationships between MR-imaged total amount of tissue removed, resection scores of specific mediobasal limbic subcompartments and clinical outcome following selective amygdalohippocampectomy." Epilepsy Res 6(1): 56-65.

Singh, N. A., C. Charlier, et al. (1998). "A novel potassium channel gene, KCNQ2, is mutated in an inherited epilepsy of newborns." Nature genetics 18(1): 25-29.

Smart, S. L., V. Lopantsev, et al. (1998). "Deletion of the $K<$ sub $>V</$ sub $>1.1$ Potassium Channel Causes Epilepsy in Mice." Neuron 20(4): 809-819. 
Spencer, S. S., P. D. Williamson, et al. (1987). "Human Hippocampal Seizure Spread Studied by Depth and Subdural Recording - the Hippocampal Commissure." Epilepsia 28(5): 479-489.

Steward, O. (1976). "Topographic organization of the projections from the entorhinal area to the hippocampal formation of the rat." J Comp Neurol 167(3): 285-314.

Steward, O. and S. A. Scoville (1976). "Cells of origin of entorhinal cortical afferents to the hippocampus and fascia dentata of the rat." J Comp Neurol 169(3): 347-370.

Stieger, K., B. Belbellaa, et al. (2009). "< i> In vivo $</ \mathrm{i}>$ gene regulation using tetracycline-regulatable systems." Advanced drug delivery reviews 61(7): 527-541.

Stojanova, V. and A. O. Rossetti (2012). "Oral topiramate as an add-on treatment for refractory status epilepticus." Acta Neurologica Scandinavica 125(2): e7-e11.

Su, H., Y. Huang, et al. (2006). "AAV serotype-1 mediates early onset of gene expression in mouse hearts and results in better therapeutic effect." Gene therapy 13(21): 1495-1502.

Sun, C., W. Sieghart, et al. (2004). "Distribution of alpha1, alpha4, gamma2, and delta subunits of GABAA receptors in hippocampal granule cells." Brain Res 1029(2): 207216.

Sun, C. S., Z. Mtchedlishvili, et al. (2007). "Selective loss of dentate hilar interneurons contributes to reduced synaptic inhibition of granule cells in an electrical stimulationbased animal model of temporal lobe epilepsy." Journal of Comparative Neurology 500(5): 876-893.

Sutula, T., G. Cascino, et al. (1989). "Mossy fiber synaptic reorganization in the epileptic human temporal lobe." Annals of neurology 26(3): 321-330.

Tallent, M. K. and C. Qiu (2008). "Somatostatin: an endogenous antiepileptic." Molecular and cellular endocrinology 286(1): 96-103.

Talley, E. M., G. Solórzano, et al. (2001). "CNS distribution of members of the two-poredomain (KCNK) potassium channel family." The Journal of Neuroscience 21(19): 74917505 . 
Tanaka, H., S. Y. Grooms, et al. (2000). "The AMPAR subunit GluR2: still front and center-stage." Brain research 886(1): 190-207.

Teitelbaum, J. S., R. J. Zatorre, et al. (1990). "Neurologic sequelae of domoic acid intoxication due to the ingestion of contaminated mussels." New England Journal of Medicine 322(25): 1781-1787.

Tolner, E. A., F. Kloosterman, et al. (2005). "Physiological changes in chronic epileptic rats are prominent in superficial layers of the medial entorhinal area." Epilepsia 46 Suppl 5: $72-81$.

Toyoda, I., M. R. Bower, et al. (2013). "Early activation of ventral hippocampus and subiculum during spontaneous seizures in a rat model of temporal lobe epilepsy." The Journal of Neuroscience 33(27): 11100-11115.

Trussell, L. O. and M. B. Jackson (1985). "Adenosine-activated potassium conductance in cultured striatal neurons." Proceedings of the National Academy of Sciences 82(14): 4857-4861.

Turski, W. A. (2000). "Pilocarpine-induced seizures in rodents--17 years on." $\underline{\text { Pol J }}$ Pharmacol 52(1): 63-65.

Van Paesschen, W., A. Connelly, et al. (1997). "The spectrum of hippocampal sclerosis: a quantitative magnetic resonance imaging study." Ann Neurol 41(1): 41-51.

van Strien, N. M., N. L. Cappaert, et al. (2009). "The anatomy of memory: an interactive overview of the parahippocampal-hippocampal network." Nat Rev Neurosci 10(4): 272282.

Vezzani, A. (2004). "Gene therapy in epilepsy." Epilepsy Curr 4(3): 87-90.

Vezzani, A., C. Schwarzer, et al. (1996). "Functional changes in somatostatin and neuropeptide $\mathrm{Y}$ containing neurons in the rat hippocampus in chronic models of limbic seizures." Epilepsy Res 26(1): 267-279.

Vezzani, A., G. Sperk, et al. (1999). "Neuropeptide Y: emerging evidence for a functional role in seizure modulation." Trends Neurosci 22(1): 25-30. 
Wang, C., C. Wang, et al. (2003). "Recombinant AAV serotype 1 transduction efficiency and tropism in the murine brain." Gene therapy 10(17): 1528-1534.

Wang, H., A. Ghosh, et al. (2008). "Therapeutic gene silencing delivered by a chemically modified small interfering RNA against mutant SOD1 slows amyotrophic lateral sclerosis progression." J Biol Chem 283(23): 15845-15852.

Wang, J. H., J. Xie, et al. (2007). "Existence of transient functional double-stranded DNA intermediates during recombinant AAV transduction." Proceedings of the National Academy of Sciences of the United States of America 104(32): 13104-13109.

Wang, L., Y. H. Liu, et al. (2008). "Time-course of neuronal death in the mouse pilocarpine model of chronic epilepsy using Fluoro-Jade C staining." Brain Research 1241: 157-167.

Wardas, J. (2002). "Neuroprotective role of adenosine in the CNS." Polish journal of pharmacology 54(4): 313-326.

Weinberg, M. S., B. L. Blake, et al. "The influence of epileptic neuropathology and prior peripheral immunity on CNS transduction by rAAV2 and rAAV5." Gene Ther 18(10): 961-968.

Wissmann, A., I. Meier, et al. (1986). "Tn10 tet operator mutations affecting Tet repressor recognition." Nucleic Acids Res 14(10): 4253-4266.

Wykes, R. C., J. H. Heeroma, et al. (2012). "Optogenetic and potassium channel gene therapy in a rodent model of focal neocortical epilepsy." Sci Transl Med 4(161): 161 ra152.

Yang, Y., M. P. Tetreault, et al. (2008). "Kruppel-like factor 5 controls keratinocyte migration via the integrin-linked kinase." J Biol Chem 283(27): 18812-18820.

YogendraSinh H Raol, I. V. L., Sabita Bandyopadhyay, Guojun Zhang, Daniel S Roberts, John H Wolfe, Shelley J Russek, and Amy R Brooks-Kayal (2006). "Enhancing GABAA Receptor al Subunit Levels in Hippocampal Dentate Gyrus Inhibits Epilepsy Development in an Animal Model of Temporal Lobe Epilepsy." Journal of Neuroscience 26(44): 11342-11346. 
Zemlan, F. P., J. J. Mulchahey, et al. (2003). "Quantification and localization of kainic acid-induced neurotoxicity employing a new biomarker of cell death: Cleaved microtubule-associated protein-tau (C-tau)." Neuroscience 121(2): 399-409.

Zuberi, S., L. Eunson, et al. (1999). "A novel mutation in the human voltage-gated potassium channel gene (Kv1. 1) associates with episodic ataxia type 1 and sometimes with partial epilepsy." Brain 122(5): 817-825. 


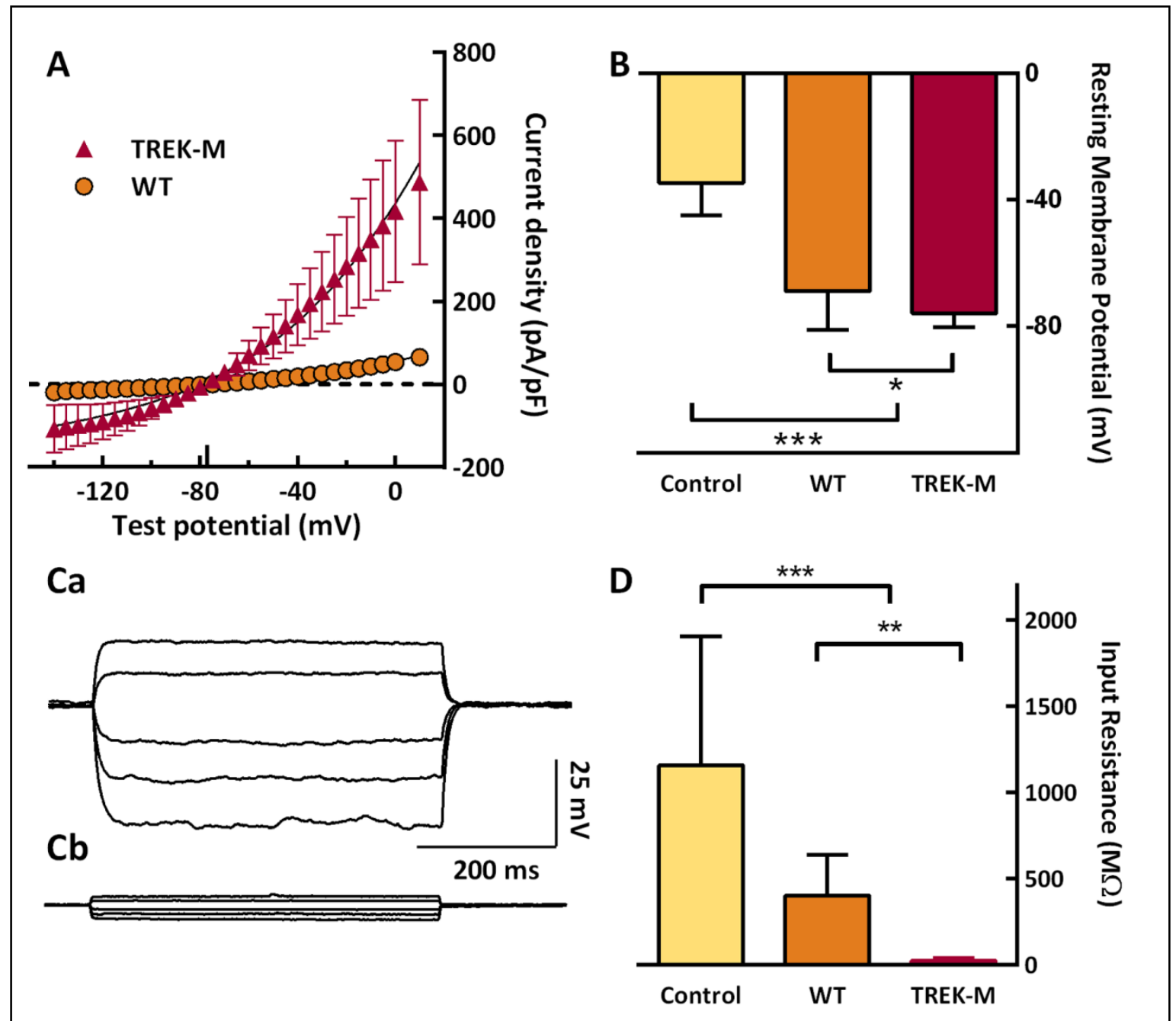




\section{Appendix figure 1: Mutations in Trek-1 increase $\mathrm{K}^{+}$leak currents, lower resting membrane potential and decrease input resistance}

(A) Expression of WT and TREK-M channel in HEK-293 cells measured by wholecell voltage clamp recording. Ionic current was normalized to the capacitance of the cell $(\mathrm{pA} / \mathrm{pF})$ and then averaged. Currents were evoked during step pulses to the indicated potentials from a holding potential of $-80 \mathrm{mV}$. The smooth lines represent fits to the data with the Goldman-Hodgkin-Katz current equation (Hille 2001). Ionic flow reversed direction at the calculated reversal potential of potassium (Erev, -77 $\mathrm{mV}$ ) and is shown as a tick on the $\mathrm{X}$-axis. TREK-M had a 7-fold higher conductance (in $\mathrm{nS} / \mathrm{pF}$ ): TREK-M, 5.3 $\pm 2.2, \mathrm{n}=15$; WT, 0.7 $\pm 0.7, \mathrm{n}=15 ; \mathrm{P}<0.0001$ ). (B) Both WT and TREK-M expression significantly altered the resting membrane potential (in $m V)$ : Control, $-35 \pm 10, n=12$; WT, $-69 \pm 21, n=18$; and TREK-M, $-76 \pm 4, n=17$. Control cells were transfected with empty vector (pcDNA3). (C) Representative current traces (Ca, WT; Cb, TREK-M) recorded at the resting membrane potential and injecting either $100,50,-50,-100$, or -150 pA current. Input resistance was calculated by fitting the resulting change in voltage with linear regression. (D)

Both WT and TREK-M also significantly reduced input resistance (in MR): Control, 1155 $\pm 716, \mathrm{n}=12$; WT, 399 $\pm 227, \mathrm{n}=18$; and TREK-M, 22 $\pm 12, \mathrm{n}=17$ ). Statistical significance estimated with Student's t-test is indicated with a single asterisk if $\mathrm{P}<0.05$, and if $\mathrm{P}<0.005$ with a triple asterisk 


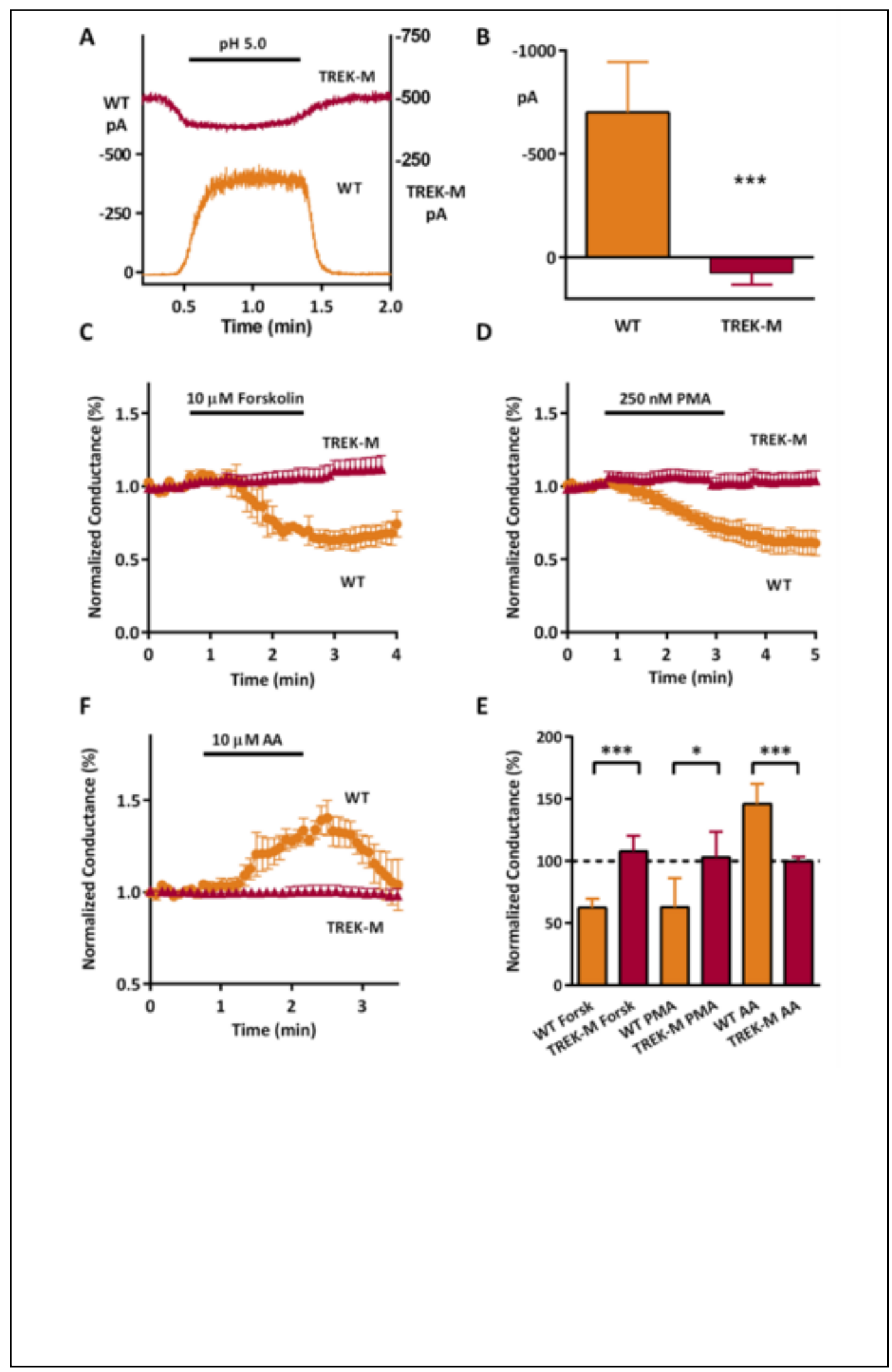




\section{Appendix figure 2: TREK-M is not regulated by intracellular acidosis or}

second messenger signaling cascades.

Ionic currents were measured using the cell excised, inside-out patch configuration at a holding potential of $0 \mathrm{mV}$ and a physiologically relevant $\mathrm{K}^{+}$ gradient $(\mathbf{A}, \mathbf{B})$. Decreasing the $\mathrm{pH}$ of the bathing solution from 7.2 to $\mathrm{pH} 5.0$ opens Trek-1 WT channels in a reversible manner. In contrast, changing the $\mathrm{pH}$ to 5.0 reduces TREK-M current amplitude. (B) Average current change induced by acidification in WT $(n=4)$ and TREK-M $(n=4)$. Whole-cell voltage-clamp recording of voltage ramps $(-140$ to $+10 \mathrm{mV})$ were used to measure changes in slope conductance induced by various second messenger cascades $(\boldsymbol{C}-\boldsymbol{F})$. Average time course after bath application of $10 \square \mathrm{M}$ forskolin (horizontal bar, $\mathrm{n}=4$ for both WT and TREK-M). (D) Bath application of $250 \mathrm{nM}$ phorbol 12myristate 13-acetate (PMA) also decreased WT Trek-1 currents but had no effect on TREK-M currents (WT, $\mathrm{n}=7$; TREK-M, n=5). (E) In contrast, perfusion with $10 \square \mathrm{M}$ arachidonic acid (AA) induced a transient stimulation of WT Trek-1 currents $(n=7)$, but had no effect on TREK-M (n=4). Peak effects and statistical analysis of the forskolin, PMA, and AA experiments are shown in panel $\mathbf{F}$. Statistical significance estimated with Student's t-test is indicated with a single asterisk if $\mathrm{P}<0.05$, and if $\mathrm{P}<0.005$ with a triple asterisk. 

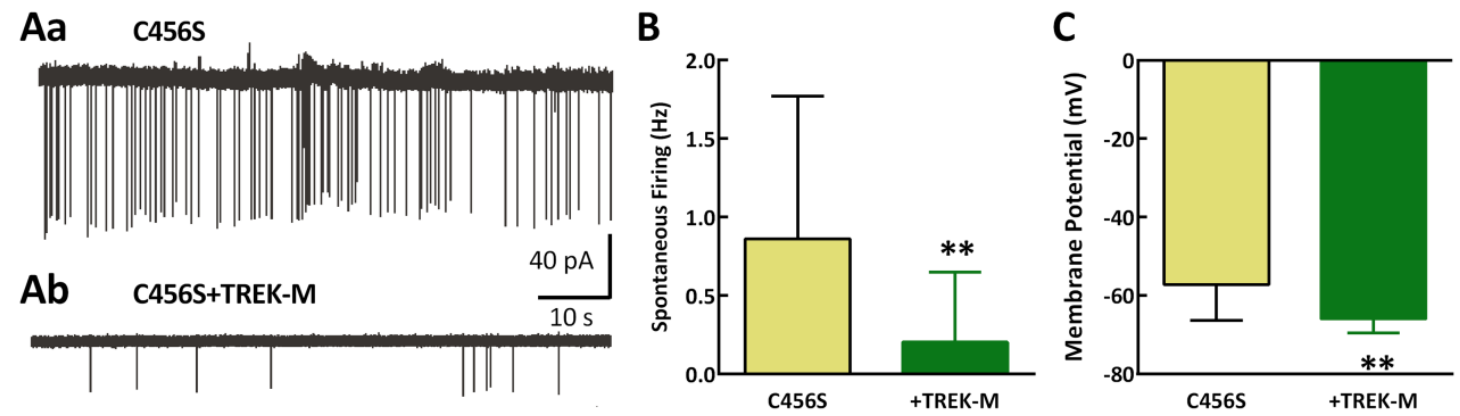

Da C456s

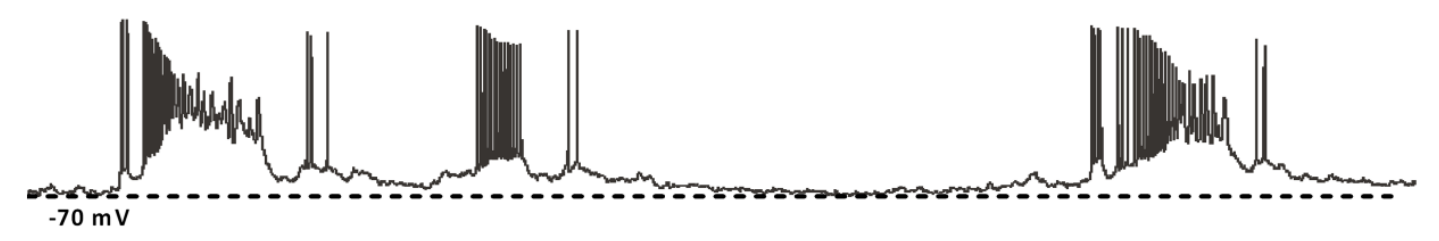

Db C456S+TREK-M

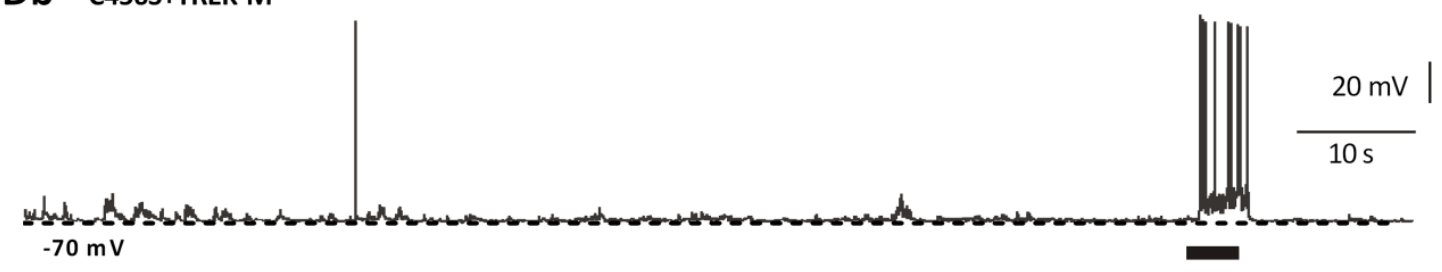




\section{Appendix Figure 3: TREK-M reduces spontaneous firing of hyperactive hippocampal neurons}

The loose cell-attached patch recording technique was used to measure spontaneous activity of mature cultures of hippocampal neurons transfected with the Cav3.2 epilepsy mutant C456S +/- TREK-M. To measure spontaneous firing without cell dialysis, we used the loose cell-attached patch recording mode $(\mathbf{A}, \mathbf{B})$. Representative traces of spontaneous activity measured in loose cell-attached patch recording mode from neurons transfected with either C456S (Aa) or C456S+TREK-M (Ab) are presented. Scale bars are shown at the ends of the traces. Note, the observed current amplitude is largely a function of access resistance. (B) Average spontaneous firing recorded in loose cell-attached patch configuration ( $\mathrm{n}$ is shown on the graph). (C) Expression of TREK-M hyperpolarized the neurons about $9 \mathrm{mV}$ from -57 to $-66 \mathrm{mV}$. The input resistance was reduced by TREK-M about 35\% compared to C456S (in MR): C456S: $188 \pm 60, n=11$; and TREK-M, 121 \pm 26 , $\mathrm{n}=5(\mathrm{P}<0.05)$. Representative traces in current-clamp mode for C456S and C456S+TREK$\mathbf{M}$ are shown in panels $\mathbf{D a}$ and $\mathbf{D b}$, respectively. Horizontal bar in $\mathbf{D b}$ represents a $+100 \mathrm{pA}$ current injection. Action potential firing was readily induced by this pulse, showing that the neuron was still capable of firing. 

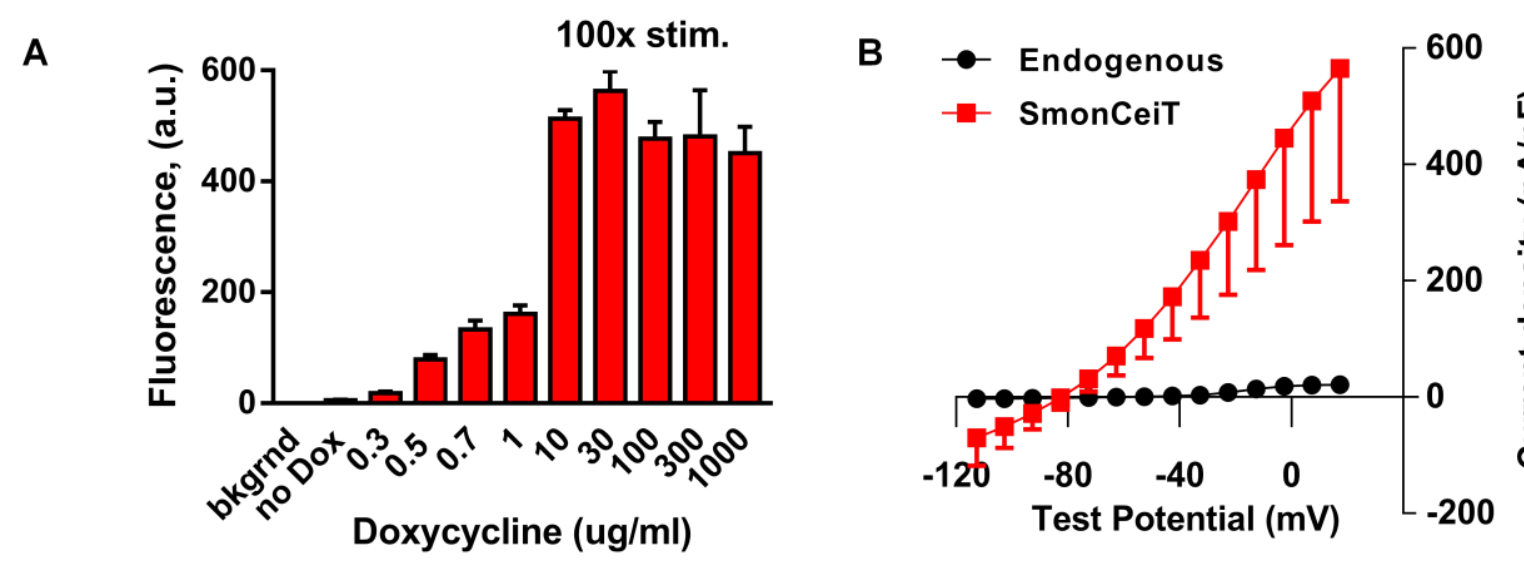

\section{Appendix figure 4: In vitro validation of SmonCeiT}

A: Doxycycline dose response in HEK-293 cells. The cells were transfected with SmonCeiT plasmid, then various doses of doxycycline were added to the culture medium. Red fluorescence in transfected cells was measured 24 hours later. Addition of concentrations greater than $10 \mu \mathrm{g} / \mathrm{ml}$ of doxycycline stimulated mCherry expression 100 -fold over no Dox control.

B. Expression of TREK-M channel red fluorescent ND7/23 neuroblastoma cells measured by whole cell patch clamp electrophysiology. ND7/23 cells were transfected with SmonCeiT and doxycycline was added to the culture medium. Currents were evoked during step pulses to the indicated potentials from a holding potential of $-80 \mathrm{mV}$. Ionic current was normalized to the capacitance if the cell $(\mathrm{pA} / \mathrm{pF})$ and then averaged. 